Der Medizinischen Fakultät

der Georg-August-Universität Göttingen

eingereicht von Prof. Dr. med. J.-H. Schiffmann

\title{
Newborn-Life-Support (NLS)-Kurse in Deutschland Analysen von Kursen zur Neugeborenenreanimation
}

\author{
INAUGURAL - DISSERTATION \\ zur Erlangung des Doktorgrades \\ der Medizinischen Fakultät \\ der Georg-August-Universität zu Göttingen \\ vorgelegt von \\ Kathrin Hanke, geb. Meier \\ aus \\ Bad Gandersheim
}

Göttingen 2010 
Diese Arbeit wurde erstellt im Jahr 2008/2009 im Klinikum Nürnberg unter Betreuung von Prof. Dr. med. J.-H. Schiffmann, Chefarzt des Zentrums für Neugeborene, Kinder und Jugendliche im Klinikum Nürnberg

Dekan:

I. Berichterstatter:

II. Berichterstatter/in:

III. Berichterstatter/in:

Tag der mündlichen Prüfung: 23. Mai 2011
Prof. Dr. med. Frömmel

Prof. Dr. med. Schiffmann

Priv.-Doz. Dr. med. Timmermann

Priv.-Doz. Dr. med. Hawighorst 


\section{Inhaltsverzeichnis}

1. Einführung in die Problemlage

1.1 Aktuelle Situation bei Reanimationsereignissen am reifen Neugeborenen 1

1.2 In Deutschland angebotene Fortbildungskurse für Neugeborenenreanimation

1.3 Newborn-Life-Support-Kurse des ERC

1.4 Verbesserung der Qualität der medizinischen Versorgung durch

Fortbildungsmaßnahmen bezüglich Neugeborenenreanimation

2. Fragestellung

3. Methodik

3.1 Teilnehmergruppe

3.2 Fragebögen

3.3 Auflistung der ausgewählten Fragen

3.4 Beschreibung der angewandten statistischen Methoden zur Auswertung der Fragebögen

3.5 Evaluation der subjektiven Effizienz des Kurses aus Sicht der Teilnehmer anhand von Beurteilungsbögen

4. Ergebnisse

4.1 Statistische Auswertung der Fragebögen: Auswertung der Gesamtergebnisse zu drei Zeitpunkten

4.2 Statistische Auswertung der Fragebögen: Auswertung der einzelnen Fragen zu den drei Zeitpunkten

4.2.1 Vergleich der Leistung vor dem Kurs (t1) versus nach dem Kurs (t2) 24 
4.2.2 Vergleich der Leistung direkt nach der Schulung (t2) versus nach sechs Monaten (t3)

4.2.3 Vergleich der Leistung vor der Schulung (t1) versus nach sechs Monaten (t3) 26

4.3 Statistische Auswertung der Fragebögen: Vergleich der Ergebnisse zwischen den verschiedenen Berufsgruppen

4.3.1 Vergleich der Ergebnisse zwischen den Berufsgruppen vor dem Kurs (t1)

4.3.2 Vergleich der Ergebnisse zwischen den Berufsgruppen direkt nach dem Kurs (t2) 28

4.3.3 Vergleich der Ergebnisse zwischen den Berufsgruppen nach sechs Monaten (t3) 29

4.4 Auswertung der subjektiven Beurteilungsbögen 30

4.4.1 Gesamtüberblick über die Kursbewertung................................ 30

4.4.2 Einzelauswertung der gestellten Fragen .................................. 31

4.4.3 Auswertung des freien Beurteilungsbogens ............................... 34

5. Diskussion

5.1 Vorgehensweisen bei der Evaluation von Reanimationskursen

5.2 Planung der Evaluation eines Reanimationskurses

5.2.1 Faktoren für einen qualitativ guten Reanimationsablauf.

5.2.2 Ziele und Lerninhalte eines Reanimationskurses 44

5.2.3 Praktisches Vorgehen bei dieser Kursevaluation 45

5.3 Diskussion der Fragestellungen 48

5.3.1 Überprüfung des Lernerfolges. 48

5.3.2 Gibt es Themengebiete, die besondere Schwierigkeiten bereiten?... 50

5.3.3 Konnte der Kurs das Selbstbewusstsein der Teilnehmer stärken und das Vertrauen in die persönlichen Fähigkeiten erhöhen?. 
5.3.4 Gibt es wiederkehrende Kritikpunkte in der subjektiven Bewertung der Kurse? 55

5.3.5 Wie kann man die Kursqualität verbessern? 58

5.3.6 In welchem Zeitraum ist eine Wiederholung des Kurses empfehlenswert?

6. Zusammenfassung

7. Anhang

Anhang 7.1 Frageboten vor dem Kurs $2008 \quad 64$

Anhang 7.2 Frageboten vor dem Kurs 2009

Anhang 7.3 Fragebogen nach dem Kurs 2008-2009 66

Anhang 7.4 Beurteilungsbogen nach dem Kurs 2008-2009 69

$\begin{array}{lll}\text { Anhang } 7.5 \text { Internet-Fragebogen 2008-2009 } & 70\end{array}$

$\begin{array}{lll}\text { Anhang 7.6 Beispielhaftes Programm eines NLS-Kurses } & 71\end{array}$

$\begin{array}{lll}\text { Anhang 7.7 Statistische Auswertung der Gesamtergebnisse } & 74\end{array}$

Anhang 7.8 Vergleich der einzelnen Fragen zu drei Zeitpunkten 76

Anhang 7.9 Berufsgruppenbezogener Vergleich der Gesamtstatistik 100

$\begin{array}{ll}\text { 8. Literaturverzeichnis } & 102\end{array}$ 


\section{Abkürzungsverzeichnis}

$\begin{array}{ll}\text { AAP } & \text { American Academy of Pediatrics } \\ \text { AHA } & \text { American Heart Association } \\ \text { ÄKN } & \text { Ärztekammer Niedersachsen } \\ \text { BLS } & \text { Basic Life Support } \\ \text { dr.wiki } & \text { Deutscher Rat für Wiederbelebung im Kindes- und Jugendalter } \\ \text { EPLS } & \text { European Pediatric Life Support } \\ \text { EPILS } & \text { European Pediatric Immediate Life Support } \\ \text { ERC } & \text { European Resuscitation Council } \\ \text { GNPI } & \text { Gesellschaft für Pädiatrische Intensivmedizin und Neonatologie } \\ \text { GRC } & \text { German Resuscitation Council } \\ \text { ILCOR } & \text { International Liaison Committee on Resuscitation } \\ \text { NLS } & \text { Newborn Life Support } \\ \text { NRP } & \text { Neonatal Resuscitation Program } \\ \text { SLÄK } & \text { Sächsische Landesärztekammer } \\ \text { UK } & \text { United Kingdom } \\ \text { USA } & \text { United States of America } \\ \text { WHO } & \text { World Health Organisation }\end{array}$




\section{Einführung in die Problemlage}

\subsection{Aktuelle Situation bei Reanimationsereignissen am reifen Neugeborenen}

Die meisten reifen Neugeborenen ab der 37. Schwangerschaftswoche, die über 2,5 $\mathrm{kg}$ wiegen, schreien und atmen innerhalb von 90 Sekunden nach der Geburt adäquat und benötigen nicht mehr Hilfe als ein wenig Stimulation durch Abtrocknen und Wärmezufuhr (Richmond 2008).

Etwa $10 \%$ aller Neugeborenen benötigen Unterstützung in der Phase der Anpassung an das extrauterine Leben; ca. 1\% aller Neugeborenen benötigt extensivere Reanimationsmaßnahmen (AHA 2005). Eine schlechte Anpassung kann nach der Phase der primären Apnoe zur Asphyxie führen. Der WHO-Report (WHO: World Health Organisation) von 2005 stellt fest, dass 8,5\% aller Kinder unter 5 Jahren weltweit an den Folgen einer perinatalen Asphyxie sterben (WHO 2005, S. 130; Bryce et al. 2005).

In Deutschland finden zwei Drittel aller Geburten in Kliniken ohne pädiatrische Abteilung statt und damit unter der Obhut eines Teams aus Gynäkologen, Hebammen und ggf. Anästhesisten. In solchen Geburtskliniken oder in Geburtshäusern kommen Reanimationssituationen zwar deutlich seltener vor, da Mütter mit potentiell kranken Kindern, bei denen kritische Situationen zu befürchten sind, meistens in Kliniken mit angeschlossener Kinderklinik entbinden. Gleichwohl treten auch in diesen Einrichtungen die vorstehend beschriebenen Notfälle auf. In Sachsen wurden im Jahr 2008 fast 50\% der Reanimationsereignisse bei Neugeborenen in Häusern der Regelversorgung verzeichnet (643 von insgesamt 1487 Neugeborenennotfällen), was etwa 2,7\% der Gesamtgeburtenzahl in diesen Krankenhäusern ausmacht (SLÄK 2008). In Niedersachsen sind die Zahlen entsprechend (894 von insgesamt 1488 Neugeborenennotfällen, das entspricht 2,3\% der Gesamtgeburtenzahl in diesen Krankenhäusern; ÄKN 2007), sodass davon auszugehen ist, dass diese beiden Bundesländer beispielhaft für Deutschland stehen können.

In $76 \%$ der Reanimationssituationen wurde ein Notfall nicht erwartet (Mitchell et al. 2002). Daher muss bei jeder Geburt ein Reanimationsteam zur Verfügung stehen. 
Dieses Team muss aber nicht zwangsläufig aus Neonatologen bestehen. Auch Hebammen oder ausgebildetes Pflegepersonal können eine genauso gute und effiziente Reanimation durchführen, wenn im Vorfeld regelmäßige Schulungen durchgeführt wurden (Chan and Hey 2006).

Bei perinatalen Notfällen in Häusern der Regelversorgung ist es üblich, ein pädiatrisches Team aus einer kooperierenden Klinik anzufordern. Dieses Team ist üblicherweise innerhalb von 20-30 Minuten vor Ort. Die initialen Reanimationsmaßnahmen müssen beim asphyktischen Neugeborenen jedoch innerhalb von 30-60 Sekunden nach der Geburt begonnen werden, um kritische Folgen für das Neugeborene verhindern zu können (AHA 2005, Kattwinkel 2006, Baskett et al. 2005, Richmond 2008) - also lange, bevor ein notfallmäßig gerufenes kinderärztliches Team in der Geburtsklinik eintreffen kann. Die Reanimation muss somit durch das geburtshilfliche Team (Hebammen und Gynäkologen und in selteneren Fällen auch Anästhesist und ggf. Schwester(n)) vor Ort begonnen werden. Es kommt folglich maßgeblich darauf an, dass das Entbindungsteam umgehend die gebotenen Maßnahmen einleitet. Aufgrund des Ausnahmecharakters der Situation ist das anwesende medizinische Personal in der Regel nicht ausreichend geübt, um auf diese Fälle angemessen zu reagieren (Verplancke et al. 2008, Jukkala and Henly 2009). Dadurch sinkt die Chance auf ein schadloses Überleben des Neugeborenen. Eine Verbesserung dieser Situation könnte dadurch erzielt werden, dass das medizinische Personal im Wege von Fortbildungen entsprechend geschult wird.

Simulationsbasierte Kurse der Reanimation von Erwachsenen werden in Deutschland bereits flächendeckend angeboten. Deren regelmäßiger Besuch ist für viele Mitarbeiter der Krankenhäuser und Gesundheitseinrichtungen bereits heute verpflichtender Bestandteil der Qualitätsmanagementprogramme. Das Angebot an Neugeborenen-Reanimationskursen ist dagegen bisher nicht nur in Deutschland, sondern auch in anderen Ländern Europas verbesserungswürdig; ein regelmäßiges Training der geburtshilflichen Abteilungen wird meist nicht durchgeführt (Ryan et al. 1998, Ergenekon et al. 2000, Jukkala et Henly 2009). 


\subsection{In Deutschland angebotene Fortbildungskurse für Neugeborenenreanimation}

Angesichts der Bedeutung eines regelmäßigen Trainings von Reanimationsmaßnahmen für den perinatalen Outcome stellt sich die Frage, ob und in welchem Umfang in Deutschland entsprechende Fortbildungen angeboten werden, um somit eine möglichst hohe Qualität der angemessenen medizinischen Versorgung für die Fälle der perinatalen Anpassungsschwierigkeiten bis hin zur Asphyxie zu gewährleisten.

Verschiedene Häuser bieten eigene hausinterne Kurse an, die von diesen Häusern selbst entwickelt werden. Die Entwicklung und logistische Durchführung eines solchen Kurses erfolgt meist im Wege von Einzelmaßnahmen und ist für Häuser ohne eigene Neonatologie äußerst schwierig. Eine gleich bleibende Qualität kann aufgrund der in der Regel bestehenden Fluktuation des medizinischen Personals nur bedingt gewährleistet werden. Eine belastbare Aussage zu der Effizienz interner Schulungsmaßnahmen würde zudem einen etwa gleichen Ausbildungsstand der Referenten, sowie vergleichbare Konzepte voraussetzen. Zur Effizienz dieser internen Maßnahmen lässt sich dadurch keine allgemeingültige Aussage treffen.

Ein Fortbildungsangebot für die Reanimation von Neugeborenen wird von der Gesellschaft für Pädiatrische Intensivmedizin und Neonatologie (GNPI) mit dem Kurs "Neugeborenennotarzt" angeboten. Der Kurs bietet zwar eine theoretische Fortbildung über die aktuellen Standards der Neugeborenenreanimation, jedoch findet kein simulationsgestütztes Training der Teilnehmer statt. Da dieser Kurs somit nur eine eingeschränkte Zielgruppe anspricht und keinerlei praktische Ausbildung bietet, erscheint dieses Kursangebot für eine weitergehende Untersuchung im Rahmen dieser Arbeit nicht geeignet.

Für simulationsbasierte Reanimationskurse an Neugeborenen existieren in Deutschland zwei Konzepte: der "Dresdner Kurs Basale Neugeborenenreanimation“ und der „Newborn Life Support (NLS) Kurs“.

Der „Dresdner Kurs Basale Neugeborenenreanimation“ wurde auf Grundlage des Neonatal Resuscitation Programms (NRP) der American Academy of Pediatrics (AAP) und der American Heart Assoziation (AHA) nach den Richtlinien der AHA (AHA 2005; Handbuch durch Kattwinkel 2006) entwickelt und findet zweimal jährlich 
im Krankenhaus Friedrichstadt in Dresden statt (Lorenz et al. 2007; siehe auch www.khdf.de/khdf/kliniken/kai/extern/akn2001/html/neugeborenen-reanimation.html; Abruf vom 16.11.2009). Dieser Kurs ist von der Sächsischen Ärztekammer zertifiziert.

Der NLS-Kurs wird von der Vereinigung Deutscher Rat für Wiederbelebung im Kindes- und Jugendalter (dr.wiki) auf Grundlage der Richtlinien des European Resuscitation Council (ERC; Baskett et al. 2005; Handbuch durch Richmond 2008) etwa zehnmal pro Jahr an verschiedenen Kliniken in Deutschland angeboten. Dieser Kurs ist von der Ärztekammer Nordrhein zertifiziert.

Eine belastbare Untersuchung eines Neugeborenen-Reanimationskurses bietet sich nur für NRP- oder NLS-Kurse an, weil diese als standardisierte Kurse mit festgelegtem Syllabus und speziell ausgebildeten Dozenten die Forderung erfüllen, eine gleich bleibende Qualität zu gewährleisten. Ein Vergleich dieser Kurse mit individuell erarbeiteten Kursen oder von derartigen Kursen untereinander ist aufgrund der zu erwartenden fehlenden Kontinuität nicht möglich, bzw. würde zu keinen belastbaren Ergebnissen führen.

In Deutschland ist die Evaluation der NRP-Fortbildungen als standardisierte Reanimationskurse des Krankenhauses Dresden Friedrichstadt grundsätzlich sinnvoll. Aufgrund der deutlich höheren Zahl an Fortbildungsangeboten des NLSKurses (das Krankenhaus Dresden Friedrichstadt bietet zwei Kurse pro Jahr an, der NLS-Kurs wird etwa zehnmal pro Jahr angeboten) und der dadurch rasch zu erreichenden aussagekräftigen Fallzahl bietet sich der deutschlandweit angebotene NLS-Kurs über dr.wiki jedoch für eine systematische Untersuchung an. Zumal die NLS-Kurse auch in den verschiedensten Regionen in Deutschland angeboten werden und somit regionale Besonderheiten zu vernachlässigen sind, dürften die bei einer Untersuchung von NLS-Kursen erzielten Ergebnissen belastbarer sein, als dieses bei einer Untersuchung der in Dresden angebotenen Kurse der Fall wäre. 


\section{$1.3 \quad$ Newborn-Life-Support-Kurse des ERC}

Im Rahmen des Angebotes an Reanimationskursen werden vom ERC/dr.wiki seit 2006 eintägige NLS-Kurse in Deutschland angeboten. Diese Kurse beziehen sich auf die einmalige Reanimationssituation eines beeinträchtigten Kindes direkt nach der Geburt und richten sich speziell an das bei einer Geburt möglicherweise involvierte Fachpersonal. So sind die Teilnehmer dieser Kurse Gynäkologen, Anästhesisten, Kinderärzte, Hebammen, Krankenschwestern und Rettungsassistenten. Die Teilnehmerzahl pro Kurs schwankt zwischen etwa 20 bis 25 Teilnehmer.

Die Instruktoren wurden hinsichtlich des pädagogischen Ausbildungskonzeptes über den ERC ausgebildet (Baskett et al. 2005, Sektion 9) und sind als Kinderärzte oder in der Kinderkrankenpflege beschäftigt. An jedem Kurstag ist eine entsprechend ausgebildete Person als Kursleitung für die Organisation und den geregelten Ablauf des Kurses verantwortlich. Zudem gibt es weitere Instruktoren, die die Teilnehmer am Vor- und Nachmittag in Kleingruppen zu je fünf bis sechs Personen in einem Instruktoren:Teilnehmerverhältnis von 1:3 unterrichten.

Während der acht Stunden Unterricht werden drei Vorträge über die Physiologie des Neugeborenen, Reanimationsablauf nach einer Geburt und über besondere Situationen im Rahmen einer Geburt gehalten. Danach werden am Vormittag das praktische Vorgehen bei Atemwegssicherung und Beatmung, Anlage eines venösen Zuganges und Medikamentengabe, sowie der praktische Reanimationsablauf mit Herzdruckmassage in vier Kleingruppen geübt. Am Nachmittag werden die Teilnehmer erneut in Kleingruppen aufgeteilt und spielen mehrfach Reanimationssituationen in vier verschiedenen Szenarien (Hausgeburt, Frühgeborenes, respiratorische Anpassungsstörung und Mekoniumaspiration) durch.

Der Ablauf des Kurses ist standardisiert. Die Kursleitung und Organisation wird nur von wenigen speziell ausgebildeten Instruktoren übernommen, um eine gleich bleibend gute Kursqualität zu gewährleisten. Die Ausbilder in den Kleingruppen sind angehalten, regelhaft nach dem vom ERC vorgegebenen didaktischen Prinzip zu unterrichten (Baskett et al. 2005, Sektion 9). 


\subsection{Verbesserung der Qualität der medizinischen Versorgung durch Fortbildungsmaßnahmen bezüglich Neugeborenenreanimation}

Nach systematischen Schulungen mithilfe des NRP-Kurses in den USA und der Türkei konnte eine Senkung von Mortalität und Morbidität in den untersuchten Krankenhäusern nachgewiesen werden (Patel et al. 2001, Duran et al. 2008a).

Obgleich das dem NLS-Kurs zu Grunde liegende Konzept schon einige Jahre in Europa praktisch erprobt ist (Singh et al. 2006, Chan and Hey 2006, Pederzini et al. 2008), liegen derzeit noch keine aussagekräftigen Studien über die Qualität und Effektivität der NLS-Kurse in Deutschland vor. Zwar ist es Teil des Kursprogramms, dass die Teilnehmer zu Beginn und am Ende eines Kurses einen Fragebogen zur Überprüfung ihrer theoretischen Kenntnisse ausfüllen und eine praktische Prüfung bestehen müssen, ehe sie das Kurszertifikat erhalten können; eine systematische Aufbereitung dieses Kurses ist bisher aber noch nicht erfolgt. 


\section{Fragestellung}

Aus den vorstehenden Ausführungen zeigt sich, dass ein regelmäßiges und effektives Training in den Reanimationsmaßnahmen für Neugeborene, insbesondere für geburtshilflich involviertes Personal, das nur in seltenen Ausnahmefällen einer Reanimationssituation unterworfen ist, unerlässlich ist, um eine möglichst hohe Qualität der entsprechenden medizinischen Versorgung für die Fälle der perinatalen Anpassungsschwierigkeiten bis hin zur Asphyxie zu gewährleisten.

An flächendeckenden Fortbildungsangeboten gibt es derzeit in Deutschland nur den sog. NLS-Kurs von dr.wiki. Ob und inwieweit diese NLS-Kurse geeignet sind, um flächendeckend einen Beitrag zur Qualitätssicherung in den vorstehend genannten Fällen zu leisten, wurde bisher noch nicht untersucht. Diese Arbeit soll diese Lücke schließen. Sofern sich im Rahmen der Untersuchung Schwachstellen des KursKonzeptes zeigen, sollen diese aufgezeigt und Lösungen angeboten werden. Insofern soll diese Arbeit auch einen Betrag dazu leisten, um die Qualität dieser Fortbildung zu verbessern. Letztlich soll ein Zeitraum festgelegt werden, innerhalb dessen eine Auffrischung der Kenntnisse empfohlen werden sollte.

Hieraus ergeben sich folgende Fragestellungen:

(a) Ist ein (anhaltender) Lernerfolg nach der Kursteilnahme festzustellen, und gibt es Unterschiede zwischen den Teilnehmern verschiedener Berufsgruppen in Vorwissen und Wissensgewinn?

Es wird davon ausgegangen, dass sich die Prozentzahl der richtigen Antworten nach dem Kurs im Vergleich zu dem Fragenbogen vor dem Kurs erhöht hat. Fraglich ist jedoch, ob die Verbesserung der Ergebnisse auch langfristig (nach sechs Monaten) noch nachweisbar ist.

Aufgrund der unterschiedlichen Ausbildungsstrukturen und der unterschiedlichen Arbeitsfelder der Teilnehmer ist von einem deutlichen Unterschied im theoretischen Vorwissen auszugehen. Es soll überprüft werden, ob diese Vermutung stimmt und ob Unterschiede im Wissenserhalt zwischen den verschiedenen Berufsgruppen nachzuweisen sind. 
(b) Gibt es Themengebiete, die besondere Schwierigkeiten bieten?

Die verschiedenen Themengebiete, die innerhalb des Kurses unterrichtet werden, bauen aufeinander auf, um letzten Endes das Flussschema der Neugeborenenreanimation zu erklären und zu untermauern. Insofern ist es sinnvoll, allen Teilnehmern gleichermaßen die Inhalte des Kurses nahe zu bringen. Sollten einige Themengebiete besondere Schwierigkeiten bieten, wäre es sinnvoll, die Präsentation oder das didaktische Herangehen zu überdenken.

(c) Konnte der Kurs das Selbstbewusstsein der Teilnehmer stärken und die Selbsteinschätzung erhöhen?

Entsprechend den Statistiken der Joint Commission on Accreditation of Healthcare Organisations (2004) ist die Hauptursache von medizinischen Fehlern mangelnde Kommunikation. Diese wiederum sei häufig auf Einschüchterung zurückzuführen. Auch nach den Studien von Verplancke et al. (2008) ist ein hohes Selbstbewusstsein und Vertrauen in die eigenen Fähigkeiten ein Faktor für einen qualitativ guten Reanimationsablauf. Daher sollte ein Reanimationskurs darauf abzielen, das Selbstbewusstsein der Teilnehmer und das Vertrauen in die eigenen Fähigkeiten zu stärken.

(d) Gibt es wiederkehrende Kritikpunkte in der subjektiven Bewertung der Kurse?

Anhand der subjektiven Bewertung des Kurses durch die Teilnehmer sollen Verbesserungspotentiale des Kurses aufgezeigt werden. Die Möglichkeit der anonymen und freien Meinungsäußerung soll den Teilnehmern die Möglichkeit geben, eigene Schwerpunkte zu setzen. Gerade die Heterogenität der teilnehmenden Berufsgruppen stellt hohe Anforderungen an die Referenten und Kursleiter, damit der Kurs von allen als effektiv und nützlich für den beruflichen Alltag angesehen wird.

\section{(e) Wie kann man die Kursqualität verbessern?}

Anhand der bisher gewonnenen Ergebnisse sollen Verbesserungsmöglichkeiten entwickelt und aufgezeigt werden. 
(f) In welchem Zeitraum ist eine Wiederholung des Kurses empfehlenswert?

Um die theoretischen Kenntnisse und praktischen Fähigkeiten aufrecht zu erhalten und ggf. zu verbessern, erscheint eine regelmäßige Auffrischung unerlässlich. Das Zertifikat des NLS-Kurses ist bisher fünf Jahre lang gültig. Hier stellt sich die Frage, ob es ausreichend ist, an einem solchen Kurs nur alle fünf Jahre teilzunehmen.

Falls dies nicht so ist, sollte je nach Evaluation des Kurses überdacht werden, ob eine Auffrischung der Kenntnisse bereits eher stattfinden sollte, zumal anderenfalls die Gefahr besteht, dass die Teilnehmer fälschlicherweise die Gültigkeit des Zertifikats mit der Aufrechterhaltung der persönlichen Fähigkeiten gleichsetzen. 


\section{Methodik}

\subsection{Teilnehmergruppe}

Die NLS-Kurse des ERC/dr.wiki sind für medizinisches Fachpersonal konzipiert. An jedem Kurs nehmen 20-25 Partizipanten teil. Im Rahmen dieser Studie wurden acht Kurse, die zwischen November 2008 und März 2009 in verschiedenen Städten und Krankenhäusern stattfanden, ausgewertet.

Insgesamt wurden Fragebögen von 186 Teilnehmern evaluiert. Dazu wurde jeder Teilnehmer gebeten, je einen Fragebogen (t1) zu Beginn des Kurses, (t2) direkt nach Abschluss des Kurses (Abschlusstest) sowie (t3) sechs Monate nach Abschluss des Kurses auszufüllen. Nach Abzug der unvollständig ausgefüllten Bögen wurden bezüglich des Vor- und Nachtestes Fragebögen von 181 Teilnehmern ausgewertet (97\% aller Teilnehmer), von diesen 181 Personen konnten 170 Fragebögen unter Berücksichtigung der Berufsgruppe ausgewertet werden (91\% aller Teilnehmer). Nach sechs Monaten beantworteten noch 57 Personen den Fragebogen (30\% aller Teilnehmer). Des Weiteren konnte eine subjektive Rückmeldung von 174 Personen erhoben und ausgewertet werden (93\% aller Teilnehmer).

Die Partizipanten der Kurse setzen sich aus Gynäkologen (21\%), Pädiatern (20\%), Anästhesisten (15\%), Kinderkrankenschwestern (18\%), Hebammen (18\%) und sonstigen Berufsgruppen wie Allgemeinärzte und Rettungsassistenten (9\%) zusammen. 


\subsection{Fragebögen}

Zur internen Evaluierung der NLS-Kurse wurde den Kursteilnehmern seit 2006 ein Fragebogen mit 17 zufällig ausgewählten Fragen vor Kursbeginn vorgelegt. Die Fragen stammen aus dem standardisierten Fragenkatalog des ERC und sollen mit „falsch“ oder „richtig“ beantwortet werden. Dieser erste Fragebogen dient dazu, den Stand der Vorkenntnisse zu evaluieren. Im Rahmen dieser Untersuchung wurden im Juni 2008 gezielt 17 Fragen aus dem Fragenkatalog des ERC ausgewählt, die den Wissenstand der Teilnehmer im Besonderen hinsichtlich der kursrelevanten Themen darstellen sollen. Anfang 2009 wurden dem ersten Fragebogen drei weitere Fragen hinzugefügt, so dass seit 2009 zu Beginn des Kurses 20 Fragen zu beantworten waren.

Nach dem Kurs beantworten die Teilnehmer im Rahmen der theoretischen und praktischen Abschlussprüfung einen Fragebogen mit 50 Fragen aus dem Fragenkatalog des ERC. In diesen 50 Fragen sind die zu Beginn des Kurses gestellten 17 Fragen enthalten (Fragen 34-50).

Um aussagekräftige Ergebnisse anhand einer ausreichend großen Gruppe von Probanten zu erzielen, werden in der Auswertung der Kurse aus dem Jahr 2008 und 2009 nur die 17 Fragen berücksichtigt, die bereits 2008 gestellt wurden. Diese sind sowohl in dem seit 2009 auf 20 Fragen erhöhten Prätest (2008 die Fragen 1-17, bzw. seit 2009 die Fragen 4-20), als auch in den 50 Fragen des Abschlusstestates enthalten (Fragen 34-50). Die Fragebögen von 2008 und 2009 sind im Anhang als Anhang 7.1, 7.2, und 7.3 eingefügt.

Nach Ablauf von sechs Monaten wurden die Kursgruppen erneut via Email angeschrieben und auf eine Internetseite verwiesen, auf der sie erneut 20 Fragen beantworten sollten. Die auszuwertenden 17 Fragen sind in diesem Fragebogen wiederum enthalten (Fragen 4-20). Eine Abschrift des Online-Fragebogens ist ebenfalls im Anhang als Anhang 7.5 eingefügt.

Die Teilnehmer gaben auf jedem Fragebogen ihren Namen an, so dass die Antworten eindeutig einem bestimmten Teilnehmer zuzuordnen waren. Insofern konnte auch die individuelle Entwicklung ausgewertet werden. 
Die im Juni 2008 für diese Untersuchung ausgewählten Fragen sollen das Wissen der Teilnehmer in den wichtigen Themenbereichen Reanimationsablauf, Atemwegsmanagement, Herz-Kreislaufreanimation und PostReanimationsmanagement abbilden. Dies sind die wichtigen Teilbereiche, die im Kurs angesprochen werden. Somit kann nach Auswertung dieser Fragen ein umfassender Eindruck über das Verständnis des Teilnehmers gewonnen werden. Ein Beispiel des Programms des NLS-Kurses ist im Anhang 7.6 nachzulesen.

Der Reanimationskurs legt einen klaren Schwerpunkt im Bereich Atemwege und Sauerstoffzufuhr beim Neugeborenen. Aus diesem Grund wurde auch bei der Auswahl der theoretischen Fragen ein entsprechend hoher Anteil an Fragen zu diesem Thema gestellt.

Alle Punkte, die in den Fragen thematisiert werden, wurden in Vorträgen oder während der praktischen Übungen im Laufe des Kurses behandelt. Die Lösungen zu den Fragebögen wurden jedoch weder nach dem Einführungstest noch nach dem Abschlusstest explizit besprochen. Die Teilnehmer bekamen auch zu keinem Zeitpunkt ein Lösungsblatt zu den gestellten Fragen. Schriftliches Material im Sinne eines Lehrbuches zu dem NLS-Kurs ist käuflich zu erwerben, wird aber den Kursteilnehmern nicht regelhaft zugesandt. Auch in diesem Lehrbuch sind die Fragen, die gestellt wurden, nicht enthalten. Die Teilnehmer mussten sich daher die Kenntnisse zur Beantwortung der Fragen durch aktives und passives Lernen im Rahmen des Kurses aneignen.

Auf diese Weise wird der zu- oder abnehmende Wissensstand der Teilnehmer ersichtlich und kann auch in Bezug auf die verschiedenen Unterrichtsbereiche klar abgebildet werden. 


\subsection{Auflistung der ausgewählten Fragen}

Folgende Fragen waren durch die Probanden mit „richtig“ oder "falsch“ zu beantwortenden:

Bereich Reanimationsablauf beim Neugeborenen:

- Der Heizstrahler ist wichtiger, als das Baby zu trocknen und einzuhüllen.

- Die initiale Reanimation eines Frühgeborenen unterscheidet sich sehr von einer Reanimation bei einem Reifgeborenen.

- Herzmassage ist sinnvoll, bevor die Lunge sich aufgebläht hat.

\section{Bereich Herz-Kreislauf beim Neugeborenen:}

- Ein notfallmäßiger venöser Zugang ist am besten über die Nabelvene zu bekommen.

- Der Haupteffekt von Adrenalin ist die Vasodilatation.

- Thoraxkompressionen sollten den Thorax $1 / 3$ des Weges bis zum Rücken eindrücken.

\section{Bereich Atemwege des Neugeborenen:}

- Bronchiallavage verbessert immer die Prognose bei Mekoniumaspiration.

- Die Atmung stoppt üblicherweise, bevor der Kreislauf versagt.

- Ein am Termin Geborenes kann mehr als $100 \mathrm{ml}$ Fruchtwasser ohne Hilfe beseitigen.

- Ein Säugling mit Herzfrequenz unter 60/min muss sofort intubiert werden.

- Systeme, die zur Beatmung von Neugeborenen benutzt werden, sollten ein Überdruckventil von 30-40 cm Wassersäule besitzen.

- Hypothermie kann Apnoen verursachen.

- Maskenbeatmung ist viel weniger effektiv, als Beatmung nach Intubation. 
- Es ist ratsam, die Reanimation bei einem Frühgeborenen mit einem niedrigeren Beatmungsdruck zu starten, als bei einem reifen Neugeborenen.

- Schnappatmung ist immer flach.

\section{Bereich Post-Reanimationsphase:}

- Alle Kinder, die bei Geburt intubiert werden, müssen auf eine Neugeborenenintensivstation übernommen werden.

- Nach einer Reanimation ist wichtig: Abnahme von Nabelschnurblut und venösem Blut zur Messung von $\mathrm{pH}$ und $\mathrm{BE}$. 


\subsection{Beschreibung der angewandten statistischen Methoden zur Auswertung der Fragebögen}

Die statistischen Auswertungen wurden mit Hilfe von SPSS für Windows, Version 17.0 (SPSS Inc., U.S.A.) durchgeführt. Die Darstellung der Prozentzahl der richtigen Antworten erfolgte über die Mittelwerte und Standardabweichungen. Die auszuwertenden Zeitpunkte wurden als t1 (Auswertung des ersten Fragebogens direkt vor dem Kurs), t2 (Auswertung des zweiten Fragebogens direkt nach dem Kurs) und t3 (Auswertung des Online-Fragebogens sechs Monate nach dem Kurs) definiert. Die Ergebnisse der einzelnen Fragen waren zu jedem Zeitpunkt klar dem jeweiligen Teilnehmer zuzuordnen, so dass verbundene Proben vorliegen.

Die Prozentzahlen der richtigen Antworten der 57 Teilnehmer zu den Zeitpunkten t1, t2 und t3 wurden mittels des Kolmogorov-Smirnov-Tests hinsichtlich ihrer Normalverteilung überprüft. Die getesteten Variablen wiesen keine Normalverteilung auf (Kolmogorov-Smirnov-Test: $\mathrm{p}<0,05$ ). Bei den Vergleichen der Stichproben wurden daher durchgehend nichtparametrische Tests für nicht-normalverteilte Stichproben herangezogen.

Der Vergleich der Beantwortung der einzelnen Fragen zu den Zeitpunkten $\mathrm{t} 1$ versus $t 2$, $t 2$ versus $t 3$ und $t 1$ versus $t 3$ wurde mit Hilfe des modifizierten Chi-Quadrat- Tests nach McNemar durchgeführt.

Der Wilcoxon-Test wurde angewandt um festzustellen, ob eine Änderung der Gesamtpunktzahl zwischen den Zeitpunkten $\mathrm{t} 1$ und $\mathrm{t} 2$, bzw. $\mathrm{t} 2$ und $\mathrm{t} 3$ sowie $\mathrm{t} 1 \mathrm{und} \mathrm{t} 3$ vorliegt.

Auf diese Weise konnte jeweils eine signifikante Veränderung (Verbesserung oder Verschlechterung) zwischen den einzelnen Zeitpunkten nachgewiesen oder ausgeschlossen werden.

Die Prozentzahlen der richtigen Antworten von 170 Teilnehmern der verschiedenen Berufsgruppen vor und nach dem Kurs wurden ebenfalls mittels des KolmogorovSmirnov-Tests hinsichtlich ihrer Normalverteilung überprüft. Während einige der getesteten Berufsgruppen keine Normalverteilung aufwiesen (Kolmogorov-SmirnovTest: $p<0,05)$, konnte für andere Variablen die Hypothese einer Normalverteilung nicht abgelehnt werden (Kolmogorov-Smirnov-Test: $p \geq 0,05$ ). 
Da jeweils mehr als zwei unabhängige, nicht-normalverteilte Stichproben verglichen wurden, wurde der $\mathrm{H}$-Test nach Kruskal und Wallis für nicht-normalverteilte Stichproben angewendet.

Bei allen durchgeführten Tests erfolgte eine zweiseitige Signifikanzüberprüfung, wobei für alle statistischen Tests ein $p$-Wert $<0,05$ als statistisch signifikant angenommen wurde.

In den grafischen Darstellungen, die ebenfalls mit SPSS erstellt wurden, wurden zur Veranschaulichung der Mediane und Quartilsabstände Kastengrafiken verwendet. Während in den Kästen der Median sowie das 25.-75. Perzentil aufgetragen sind, entsprechen die T-Balken dem kleinsten und größten Wert, sofern diese keine Ausreißer bzw. Extremwerte sind. Die Ausreißer sind dabei Werte, die zwischen 1,53 Kastenlängen außerhalb des Kastens liegen; sie sind in den Grafiken als Kreise dargestellt.

Die Anteile der richtigen Antworten zu den Fragen 1-17 wurden grafisch mit Hilfe von Balkendiagrammen dargestellt.

Hinsichtlich der statistischen Bearbeitung erfolgte eine Beratung durch Frau Dr. T. Kottmann (Statistik-Service/Medizinische Wissenschaftsberatung; Akazienweg 22 32584 Löhne). 


\subsection{Evaluation der subjektiven Effizienz des Kurses aus Sicht der Teilnehmer anhand von Beurteilungsbögen}

Am Ende eines jeden Kurses wurden die Teilnehmer gebeten, in anonymer Form, aber unter Angabe der Berufsbezeichnung, eine subjektive Rückmeldung bezüglich des Kurses zu geben. Durch eine anonyme Umfrage sollte eine unverfälschte Rückmeldung bezüglich der Kursqualität gewährleistet werden. Das Wissen um die Berufsgruppe des Teilnehmers erschien notwendig, um eventuell vorhandenes Vorwissen oder Spezialwissen oder besondere Erwartungen besser einschätzen zu können. In dieser Studie wurden 174 Beurteilungsbögen aus 8 Kursen ausgewertet. Der Aufbau der Beurteilungsbögen kann im Anhang 7.4 nachgelesen werden.

Die Partizipanten wurden gebeten, eine subjektive Benotung des Kurses hinsichtlich des individuellen praktischen Nutzens für die tägliche Arbeit und des Informationsgehaltes der Fortbildung abzugeben. Des Weiteren wurde abgefragt, ob der Teilnehmer angeregt wurde, Aspekte seiner medizinischen Tätigkeit zu überdenken oder zu ändern. Die Referenten sollten bezüglich ihrer Fachkompetenz und verständlichen Darstellung der Themen beurteilt werden. Unter dem Punkt "Organisation“ wurde abgefragt, ob die Dauer des Kurses als angemessen empfunden wurde. Letztlich wurden die Teilnehmer um einen Gesamteindruck gebeten, und die Frage gestellt, ob sich ihre persönlichen Ziele und Erwartungen erfüllt hätten. Am Ende eines jeden Fragebogens hatte jeder Teilnehmer die Möglichkeit, frei formuliert positive und negative Kritik zu äußern und Verbesserungsvorschläge zu erstellen.

In den Beurteilungsbögen konnten Punkte auf einer Skala von ++ bis -- vergeben werden. Diese Skala wurde dann zur besseren Auswertbarkeit in Punkte von Fünf bis Eins entsprechend dem folgenden Schema umgewandelt:

\begin{tabular}{|c|l|c|}
\hline Skala & Bedeutung & Punktzahl in der Auswertung \\
\hline++ & Sehr gut / trifft gut zu & 5 \\
\hline+ & & 4 \\
\hline 0 & Mittelmäßig & 3 \\
\hline- & & 2 \\
\hline-- & schlecht / trifft in überhaupt nicht zu & 1 \\
\hline
\end{tabular}




\section{Ergebnisse}

\subsection{Statistische Auswertung der Fragebögen: Auswertung der Gesamtergebnisse zu drei Zeitpunkten}

Ziel der Auswertung der Gesamtergebnisse war es festzustellen, ob der Wissensstand der Teilnehmer im Rahmen der Schulung wesentlich steigt und ob dieses Wissen über mindestens sechs Monate nach Kursende erhalten werden kann.

Zur Auswertung des Kurses hinsichtlich der Änderungen des Wissensstandes (im Sinne einer Änderung des Prozentsatzes an richtigen Antworten auf die 17 ausgewerteten Fragen) vor und nach der Schulung (zu den Zeitpunkten t1, t2 und t3) wurden nur die 57 Kandidaten einbezogen, die den Fragebogen zu allen drei Zeitpunkten ausgefüllt haben. Dass diese Probandengruppe repräsentativ für die Gesamtheit der Teilnehmer stehen kann, zeigt sich im Vergleich: Wir haben die Gesamtgruppe (181 Teilnehmer), sowie die Teilgruppe von 57 Teilnehmern zu den Zeitpunkten t1 (Vor dem Kurs) und t2 (direkt nach dem Kurs) hinsichtlich Mittelwert, Median und Standardabweichung ausgewertet. Es sind keine wesentlichen Unterschiede zwischen den beiden Gruppen feststellbar (siehe Tabelle 1). Diese Feststellung lässt sich auch im Vergleich der Grafiken 1 und 2 visuell nachvollziehen.

\begin{tabular}{|l|l|l|l|l|}
\hline & $\begin{array}{l}\text { Alle } \\
\text { Teilnehmer } \\
\text { vor dem } \\
\text { Kurs }\end{array}$ & $\begin{array}{l}\text { Teilgruppe } \\
\text { vor dem } \\
\text { Kurs }\end{array}$ & $\begin{array}{l}\text { Alle } \\
\text { Teilnehmer } \\
\text { direkt nach } \\
\text { dem Kurs }\end{array}$ & $\begin{array}{l}\text { Teilgruppe } \\
\text { direkt nach } \\
\text { dem Kurs }\end{array}$ \\
\hline N (Teilnehmerzahl) & 181 & 57 & 181 & 57 \\
Mittelwert (\%) & 80,99 & 81,42 & 92,33 & 92,67 \\
Median (\%) & 82,35 & 82,35 & 94,12 & 94,12 \\
Standardabweichung (\%) & 10,54 & 11,33 & 8,29 & 7,88 \\
\hline
\end{tabular}

Tabelle 1: Vergleich der Ergebnisse von 181 und 57 Probanden vor und direkt nach dem Kurs 


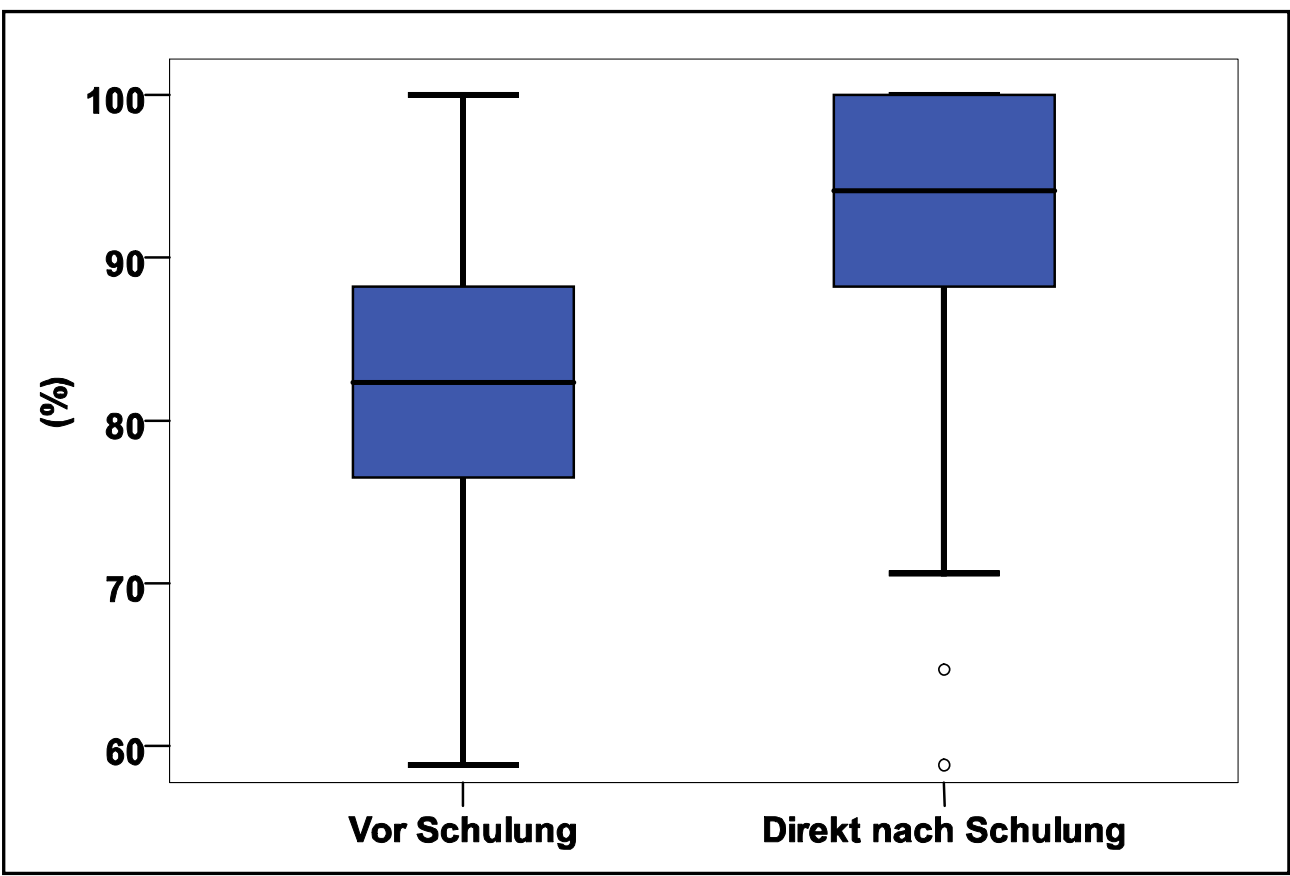

Grafik 1: Auswertung der Gesamtergebnisse von 181 Teilnehmern vor und nach dem Kurs

Im Folgenden wird also zum Vergleich der drei Zeitpunkte t1 (vor dem Kurs), t2 (direkt nach dem Kurs und $\mathrm{t} 3$ (sechs Monate nach dem Kurs) nur die Gruppe der 57 Teilnehmer besprochen, von der Daten zu allen drei Zeitpunkten zur Verfügung stehen.

Nach Berechnungen über den Wilcoxon-Test lassen sich in dieser Gruppe signifikante Änderungen (mit jeweils $p<0,001$ ) der Ränge im Vergleich von $\mathrm{t} 1$ und $\mathrm{t} 2$, t 1 und $\mathrm{t} 3$ sowie $\mathrm{t} 2$ und $\mathrm{t} 3$ nachweisen. In Zusammenschau mit den erhobenen Daten lässt sich somit eine signifikante Verbesserung des Wissensstandes nach dem Kurs feststellen $(p<0,001)$. Der Mittelwert der richtigen Antworten steigt von $81 \%$ auf $93 \%$. In den folgenden sechs Monaten kommt es zu einem signifikanten Wissensverlust $(p<0,001)$. Der Mittelwert der richtigen Antworten sinkt von $93 \%$ auf $87 \%$ ab. Trotzdem ist das Wissensniveau auch sechs Monate nach dem Kurs höher, als es vor dem Kurs war $(p<0,001$; siehe Tabelle 2$)$. 


\begin{tabular}{|l|l|l|l|}
\hline & $\begin{array}{l}\text { Richtige } \\
\text { Antworten vor dem } \\
\text { Kurs }\end{array}$ & $\begin{array}{l}\text { Richtige } \\
\text { Antworten direkt } \\
\text { nach dem Kurs }\end{array}$ & $\begin{array}{l}\text { Richtige } \\
\text { Antworten nach 6 } \\
\text { Monaten }\end{array}$ \\
\hline N (Teilnehmerzahl) & 57 & 57 & 57 \\
Mittelwert (\%) & $\mathbf{8 1 , 4 2}$ & $\mathbf{9 2 , 6 7}$ & $\mathbf{8 6 , 8 9}$ \\
Median (\%) & 82,35 & 94,12 & 88,24 \\
Standardabweichung (\%) & $\mathbf{1 1 , 3 2 5}$ & $\mathbf{7 , 8 8 3}$ & $\mathbf{9 , 1 0 1}$ \\
$\begin{array}{l}\text { Minimum (auf den einzelnen } \\
\text { Probanden bezogen) (\%) }\end{array}$ & 47 & 59 & 59 \\
Maximum (auf den einzelnen & 100 & 100 & 100 \\
Probanden bezogen) (\%) & 73,53 & 88,24 & 82,35 \\
Perzentil 25 (\%) & 82,35 & 94,12 & 88,24 \\
Perzentil 50 (\%) & 88,24 & 100,00 & 94,12 \\
Perzentil 75 (\%) & & \\
\hline
\end{tabular}

Tabelle 2: Auswertung der Gesamtergebnisse der Teilgruppe von 57 Probanden zu drei Zeitpunkten

Die Gesamtheit der ausgewerteten Daten zu den drei Zeitpunkten wird hier noch einmal anhand von Kastengrafiken dargestellt (siehe Grafik 2). Der obere und untere Rand der Kästen stellt jeweils das 25. bzw. 75. Perzentil dar. Der Median ist durch den Querstrich innerhalb des Kastens dargestellt. Die T-Balken entsprechen der Spannweite, Ausreißer werden mit einem Kreis (o) gekennzeichnet.

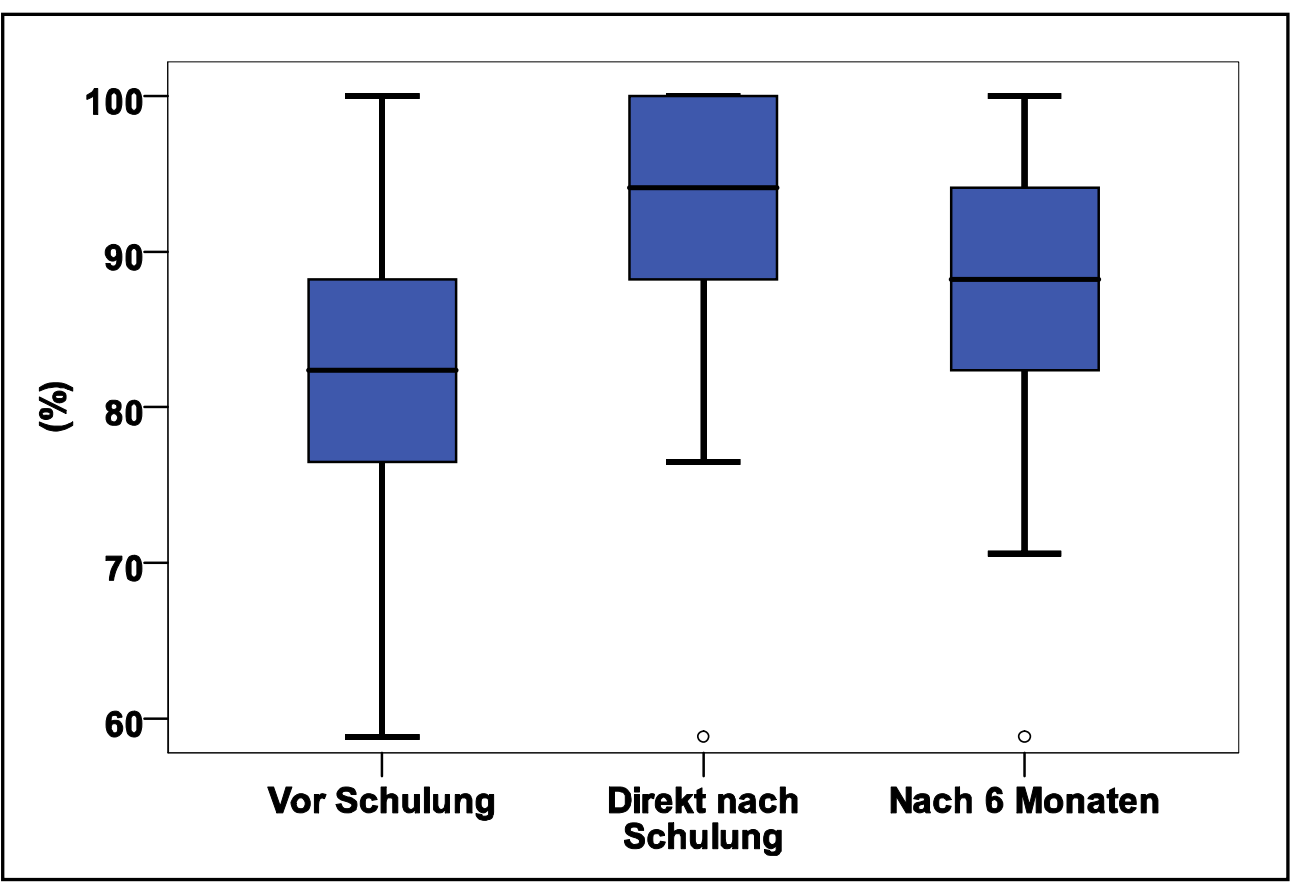

Grafik 2: Vergleich der Ergebnisse von 57 Probanden zu den evaluierten drei Zeitpunkten 


\subsection{Statistische Auswertung der Fragebögen: Auswertung der einzelnen Fragen zu den drei Zeitpunkten}

Auch in der Auswertung der einzelnen Fragen sind wiederum nur die Antworten der 57 Teilnehmer evaluiert worden, von denen zu drei Zeitpunkten Daten vorlagen. Ziel der Auswertung war es, Fragestellungen oder Themenbereiche herauszurücken, die den Teilnehmern besondere Schwierigkeiten bereiten.

Wie bereits im oberen Abschnitt erwähnt, ist nach der initialen Verbesserung des Wissensstandes ein Leistungsabfall zu erkennen. Die Fragen wurden bereits vor der Schulung zu mehr als $81 \%$ richtig beantwortet. Im Verlauf des Kurses stieg der Wissensstand auf durchschnittlich 93\% und sank sechs Monate nach dem Kurs nicht unter das Niveau ab, das bereits vor dem Kurs bestand, sondern hielt sich bei etwa 87\% richtig beantworteter Fragen. Auch in der Auswertung der einzelnen Fragen stellt sich diese Tendenz dar und soll hier nicht im Einzelnen aufgelistet werden. Die differenzierte Einzelauswertung der Fragen ist im Anhang 7.8 nachzulesen.

Zwei Fragen weichen von der oben genannten Tendenz ab, sie werden im Zeitverlauf zunehmend häufig falsch beantwortet und werden im Folgenden dargestellt:

\section{Frage 3: Der Haupteffekt von Adrenalin ist die Vasodilatation}

Die Zahl der richtigen Antworten vor der Schulung ist $86 \%$ und sinkt im Verlaufe des Kurses auf $80,7 \%$. Sechs Monate nach dem Kurs beantworten nur noch $75 \%$ der Teilnehmer diese Frage richtig.

\section{Frage 16: Schnappatmung ist immer flach}

Die Zahl der richtigen Antworten vor der Schulung ist $81 \%$ und sinkt im Verlaufe des Kurses auf $77 \%$. Sechs Monate nach dem Kurs beantworten nur noch $72 \%$ der Teilnehmer diese Frage richtig. 
Vier weitere Fragen scheinen als besonders schwierig empfunden zu werden und werden vor dem Kurs zu weniger als 73,5\% richtig beantwortet (das entspricht einer Rate an richtigen Antworten unterhalb des 25. Perzentils):

Frage 4: Bronchiallavage verbessert immer die Prognose bei Mekoniumaspiration

Diese Frage wird vor Kursbeginn nur von 56\% (<25. Perzentil) der Teilnehmer richtig beantwortet, im Verlauf steigt die Zahl der richtigen Antworten auf $77 \%$ (<25. Perzentil), fällt aber nach sechs Monaten wieder auf 70\% (<25. Perzentil) ab. Dieser Verlauf entspricht der zuvor festgestellten Tendenz des Wissenszuwachses durch die Schulung und des darauf folgenden sinkenden Wissenstandes auf niedrigem Niveau.

Frage 7: Ein am Termin Geborenes kann mehr als 100 ml Fruchtwasser ohne Hilfe beseitigen

Diese Frage wird vor Kursbeginn nur von 63\% (<25. Perzentil) der Teilnehmer richtig beantwortet, im Verlauf steigt die Zahl der richtigen Antworten auf 86\% (<25. Perzentil), fällt aber nach sechs Monaten wieder auf 84\% (<50. Perzentil) ab.

Frage 10: Systeme, die zur Beatmung von Neugeborenen benutzt werden, sollte ein Überdruckventil von $30-40 \mathrm{~cm}$ Wassersäule besitzen

Diese Frage wird vor Kursbeginn nur von 65\% (<25. Perzentil) der Teilnehmer richtig beantwortet, im Verlauf steigt die Zahl der richtigen Antworten auf 100\% (75. Perzentil), fällt aber nach sechs Monaten wieder auf $77 \%(<25$. Perzentil) ab.

Frage 15: Die initiale Reanimation eines Frühgeborenen unterscheidet sich sehr von der Reanimation eines Reifgeborenen

Diese Frage wird vor Kursbeginn nur von 72\% (<25. Perzentil) der Teilnehmer richtig beantwortet, im Verlauf steigt die Zahl der richtigen Antworten auf 93\% (<50. Perzentil), fält aber nach sechs Monaten wieder auf 82\% (25. Perzentil) ab.

Die folgenden Diagramme stellen die Prozentzahl der richtigen Antworten bei den 17 ausgewerteten Fragen nochmals graphisch dar (siehe Diagramm 3 bis 5). 


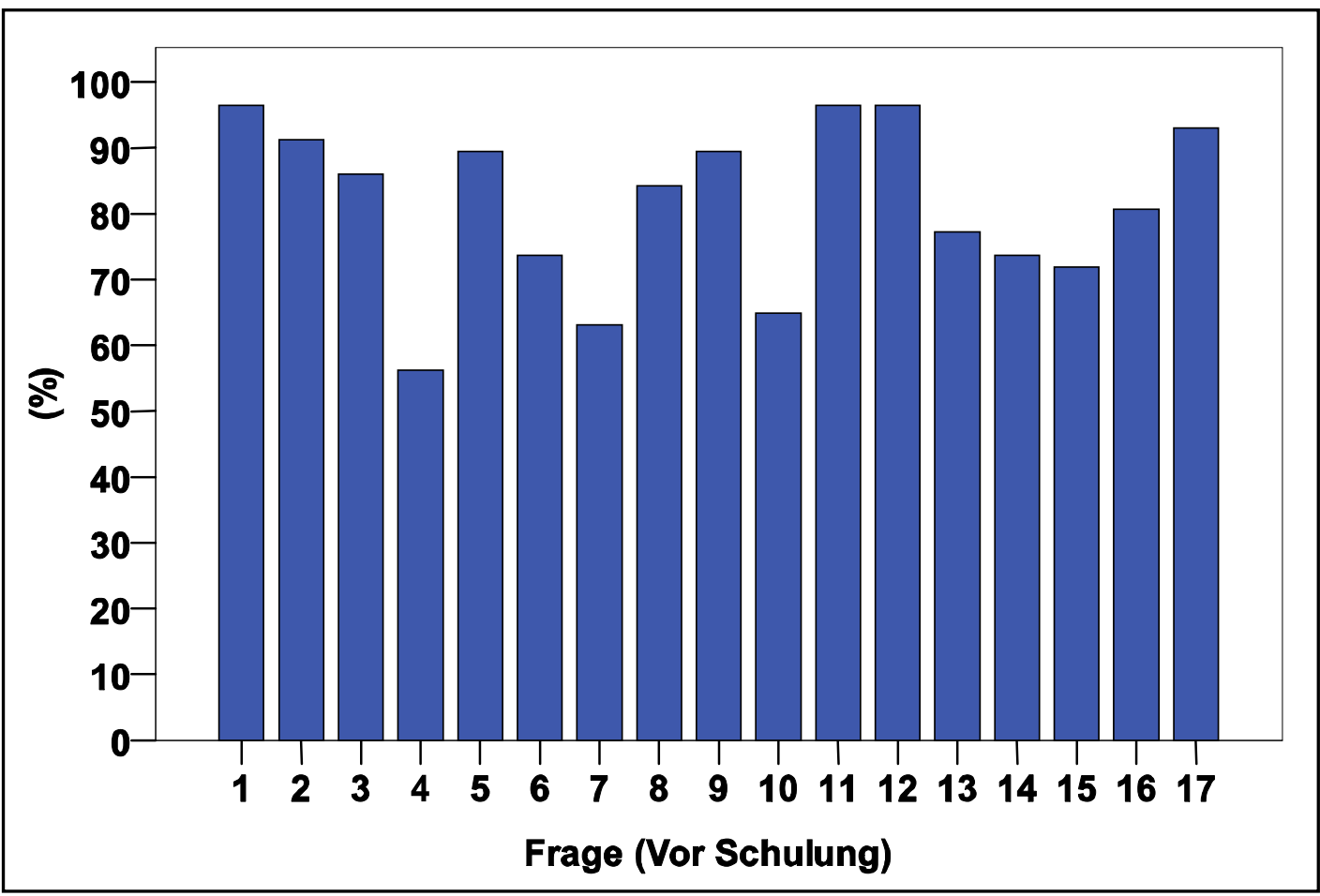

Grafik 3: Vor der Schulung: richtige Antworten in Prozent

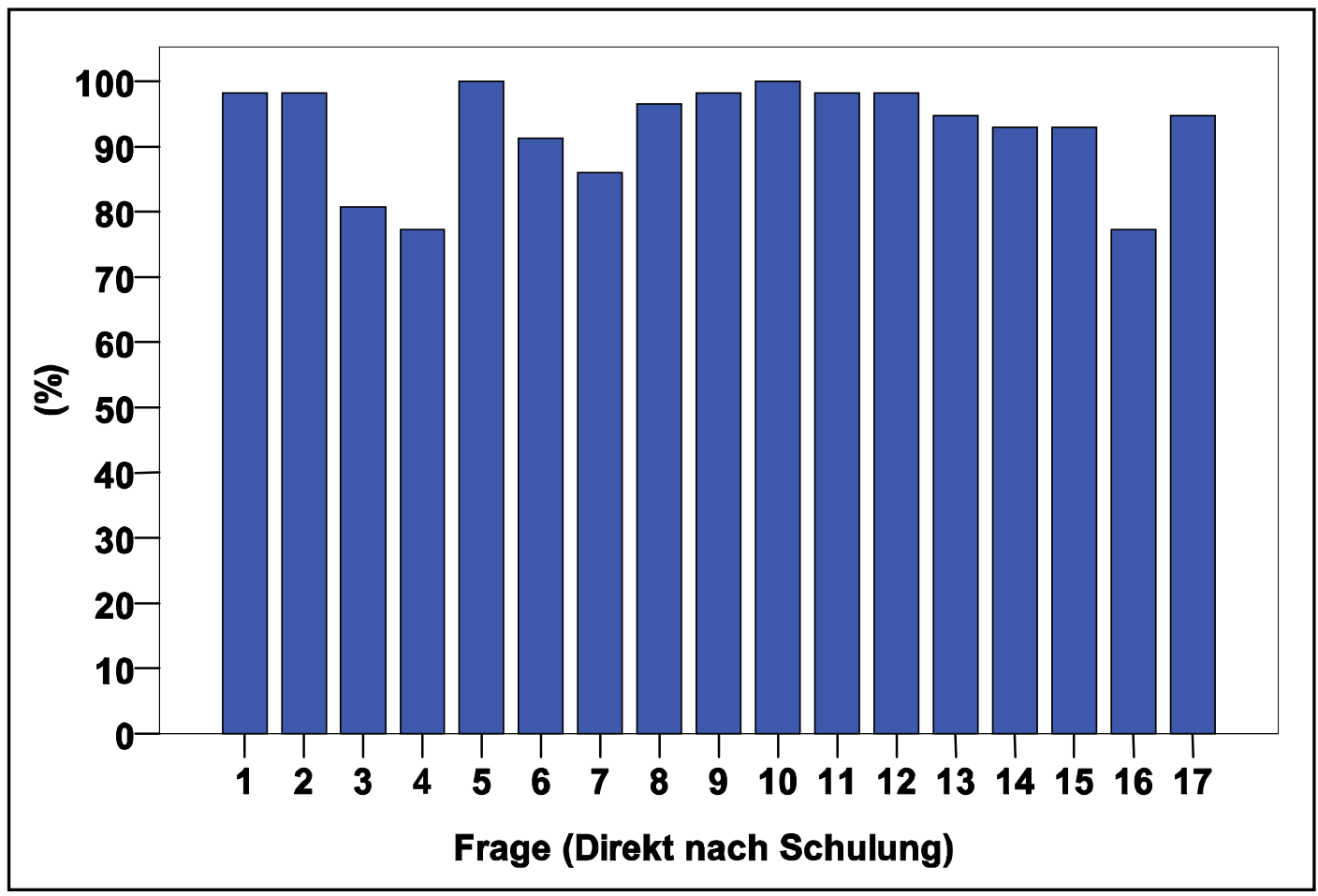

Grafik 4: Direkt nach dem Kurs: richtige Antworten in Prozent 


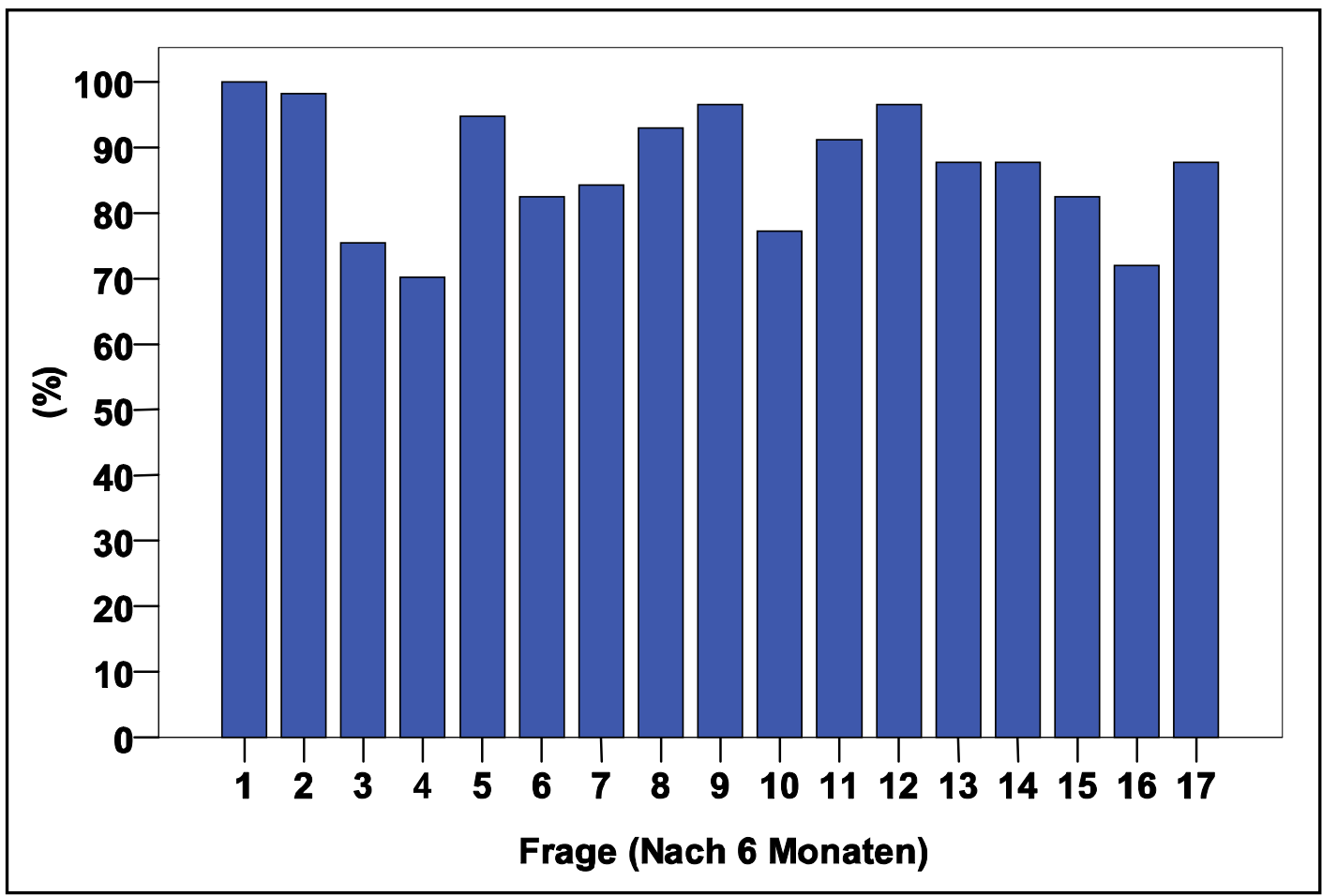

Grafik 5: Nach sechs Monaten: richtige Antworten in Prozent

\subsubsection{Vergleich der Leistung vor dem Kurs (t1) versus nach dem Kurs (t2)}

In diesem Vergleich wurden nur die 57 Fälle herangezogen, von denen Daten zu allen drei Zeitpunkten vorhanden waren, um einen reellen Vergleich zwischen den drei Zeitpunkten durchführen zu können. Die einzelnen Ergebnisse der statistischen Berechnungen können im Anhang 7.8 nachgelesen werden.

Im Vergleich der Ergebnisse vor versus nach der Schulung konnte bei sieben Fragen eine signifikante Veränderung berechnet werden, wobei diese Veränderungen immer im Sinne einer Verbesserung zu werten waren. Die Fragen und die Prozentsätze der richtigen Antworten werden in Tabelle 3 dargestellt: 


\begin{tabular}{|l|l|l|l|}
\hline Frage & $\begin{array}{l}\text { Prozentzahl der } \\
\text { richtigen Antworten } \\
\text { vor dem Kurs (t1) }\end{array}$ & $\begin{array}{l}\text { Prozentzahl der } \\
\text { richtigen Antworten } \\
\text { nach dem Kurs (t2) }\end{array}$ & $\begin{array}{l}\text { Signifikanz } \\
\text { (p-Wert) }\end{array}$ \\
\hline F4 & $56,1 \%$ & $77,2 \%$ & 0,004 \\
\hline F5 & $89,5 \%$ & $100 \%$ & $\star$ \\
\hline F6 & $73,7 \%$ & $91,2 \%$ & 0,006 \\
\hline F7 & $63,2 \%$ & $86 \%$ & 0,007 \\
\hline F8 & $84,2 \%$ & $96,5 \%$ & 0,016 \\
\hline F10 & $64,9 \%$ & $100 \%$ & $*$ \\
\hline F13 & $77,2 \%$ & $94,7 \%$ & 0,006 \\
\hline F14 & $73,7 \%$ & $93 \%$ & 0,001 \\
\hline F15 & $71,9 \%$ & $93 \%$ & 0,012 \\
\hline
\end{tabular}

Tabelle 3: Signifikante Veränderungen in der Anzahl der richtigen Antworten zwischen t1 und t2

* Nach dem Kurs wurde die Frage von allen Teilnehmern richtig beantwortet, so dass dieser Wert eine Konstante ist. Ein p-Wert kann daher nicht berechnet werden.

Ein auffälliges Ergebnis zeigt des Weiteren Frage 3 (Der Haupteffekt von Adrenalin ist die Vasodilatation):

Von den Teilnehmern, die diese Frage vor dem Kurs falsch beantwortet haben, konnte keiner nach der Schulung die Frage richtig beantworten. Drei Probanden, die die Frage vor dem Kurs noch richtig beantworteten, gaben nach dem Kurs falsche Antworten.

\subsubsection{Vergleich der Leistung direkt nach der Schulung (t2) versus nach sechs Monaten (t3)}

In der Auswertung der einzelnen Fragen konnte keine signifikante Änderung im Prozentsatz der richtigen Antworten nach der Schulung im Vergleich zu sechs Monate später nachgewiesen werden. Es findet sich jedoch in allen Fragen ein mehr oder weniger ausgeprägter Trend zur Verschlechterung. Dieser Trend wird erst bei der Auswertung der Gesamtergebnisse aufgrund der höheren Fallzahl sichtbar (siehe Tabelle 2). 


\subsubsection{Vergleich der Leistung vor der Schulung (t1) versus nach sechs Monaten} (t3)

In den hier verglichenen 57 Fällen konnte bei drei Fragen eine signifikante Veränderung festgestellt werden. Bei dieser Veränderung handelt es sich in jedem Fall um eine Verbesserung der Ergebnisse (siehe Tabelle 4). Es zeigt sich aber auch, dass der im Kurs erreichte Wissensgewinn (bei neun Fragen eine signifikante Verbesserung) nicht $\mathrm{zu}$ halten ist und bereits nach sechs Monaten ein Wissensverlust festzustellen ist.

\begin{tabular}{|l|l|l|l|}
\hline Frage & $\begin{array}{l}\text { Prozentzahl der } \\
\text { richtigen Antworten } \\
\text { vor dem Kurs (t1) }\end{array}$ & $\begin{array}{l}\text { Prozentzahl der } \\
\text { richtigen Antworten } \\
\text { nach 6 Monaten (t3) }\end{array}$ & $\begin{array}{l}\text { Signifikanz } \\
\text { (p-Wert) }\end{array}$ \\
\hline F4 & $56,1 \%$ & $70,2 \%$ & 0,021 \\
\hline F7 & $63,2 \%$ & $84,2 \%$ & 0,008 \\
\hline F14 & $73,7 \%$ & $87,7 \%$ & 0,021 \\
\hline
\end{tabular}

Tabelle 4: Signifikante Veränderungen in der Anzahl der richtigen Antworten zwischen t1 und t3

Bei allen anderen Fragen sind ebenfalls Veränderungen im Prozentsatz der richtigen Antworten festzustellen, nur können diese Veränderungen aufgrund der geringen Anzahl an ausgewerteten Probanden $(n=57)$ nicht als mathematisch signifikant bewertet werden.

Die Frage 3 (Der Haupteffekt von Adrenalin ist die Vasodilatation) zeigt erneut ein auffälliges Ergebnis: Von den Teilnehmern, die diese Frage vor dem Kurs falsch beantwortet haben, beantwortete ein Teilnehmer diese Frage nach sechs Monaten richtig. Sieben Probanden, die die Frage vor dem Kurs noch richtig beantworteten, gaben nach sechs Monaten eine falsche Antwort.

Bei der Frage 16 (Schnappatmung ist immer flach) kann keine signifikante Veränderung nachgewiesen werden $(p=0,302)$. Es fält aber auf, dass zehn Probanden, die diese Frage vor der Schulung richtig beantwortet haben, sechs Monate später eine falsche Antwort geben. Dagegen ist nur bei fünf Probanden ein Wissenszuwachs festzustellen. 


\subsection{Statistische Auswertung der Fragebögen: Vergleich der Ergebnisse zwischen den verschiedenen Berufsgruppen}

Die Ergebnisse der einzelnen Berufsgruppen wurden verglichen, um Differenzen im Vorwissen oder Wissenserhalt der Teilnehmer beurteilen zu können. Für eine belastbare statistische Auswertung der Berufsgruppen hinsichtlich der einzelnen Fragen war die gewonnene Datenmenge zu gering. Dennoch kann nach Evaluation der Gesamtergebnisse, aufgeschlüsselt nach Berufsgruppen, eine Aussage darüber getroffen werden, ob die Berufsgruppen hinsichtlich des Lerneffektes deutlich differieren und ob sich die Unterschiede zwischen den Berufsgruppen auf den subjektiven Gewinn des Einzelnen auswirken.

Der Vergleich der Punktezahl, aufgeschlüsselt nach Berufsgruppen vor dem Kurs und direkt nach dem Kurs, wurde mit 170 Teilnehmern durchgeführt, sechs Monate nach der Schulung wurden die Daten von 57 Teilnehmern ausgewertet.

\subsubsection{Vergleich der Ergebnisse zwischen den Berufsgruppen vor dem Kurs (t1)}

Die Gruppen sind nicht normalverteilt, daher wurde der $\mathrm{H}$-Test nach Kruskal und Wallis angewendet, um Unterschiede zwischen den Teilnehmern, aufgeteilt nach Berufsgruppen, nachzuweisen. Es konnte ein signifikanter Unterschied nachgewiesen werden $(p<0,001)$. Hebammen und Kinderkrankenschwestern hatten vor dem Kurs deutlich schlechtere Gesamtergebnisse, als Gynäkologen und Anästhesisten. Pädiater hatten bereits vor dem Kurs einen Wissensvorsprung (siehe Tabelle 5).

\begin{tabular}{|l|l|l|l|l|l|}
\hline Berufsgruppe & $\begin{array}{l}\text { Mittelwert } \\
(\%)\end{array}$ & $\begin{array}{l}\text { Standard- } \\
\text { abweichung } \\
(\%)\end{array}$ & $\begin{array}{l}\text { Standardfehler } \\
\text { des Mittelwertes } \\
(\%)\end{array}$ & $\begin{array}{l}\text { Median } \\
(\%)\end{array}$ & $\begin{array}{l}\mathbf{N} \\
(\text { Teilnehmerz } \\
\text { ahl) }\end{array}$ \\
\hline Gynäkologen & 82,18 & 9,873 & 1,693 & 82,35 & 34 \\
Pädiater & 87,71 & 8,302 & 1,238 & 88,24 & 45 \\
Anästhesisten & 82,35 & 9,843 & 1,930 & 82,35 & 26 \\
$\begin{array}{l}\text { Hebammen } \\
\text { Kinderkranken- } \\
\text { schwestern } \\
\text { Insgesamt }\end{array}$ & 76,29 & 9,918 & 1,727 & 76,47 & 33 \\
\hline
\end{tabular}

Tabelle 5: Vergleich der Ergebnisse der unterschiedlichen Berufsgruppen vor dem Kurs 


\subsubsection{Vergleich der Ergebnisse zwischen den Berufsgruppen direkt nach dem Kurs (t2)}

Wiederum wurde der H-Test nach Kruskal/Wallis angewandt, um Unterschiede zwischen den Berufsgruppen nachzuweisen. Es konnten erneut signifikante Unterschiede zwischen den Berufsgruppen nachgewiesen werden $(p<0,001)$. Der Wissensstand war bei allen Berufsgruppen gleichermaßen angestiegen, die Pädiater zeigten weiterhin einen Wissensvorsprung vor den anderen Berufsgruppen (siehe Tabelle 6).

\begin{tabular}{|c|c|c|c|c|c|}
\hline Berufsgruppe & \begin{tabular}{|l} 
Mittelwert \\
$(\%)$
\end{tabular} & $\begin{array}{l}\text { Standard- } \\
\text { abweichung } \\
(\%)\end{array}$ & $\begin{array}{l}\text { Standardfehler } \\
\text { des Mittelwertes } \\
(\%)\end{array}$ & \begin{tabular}{|l} 
Median \\
$(\%)$
\end{tabular} & $\begin{array}{l}\mathbf{N} \\
\text { (Teilnehmerz } \\
\text { ahl) }\end{array}$ \\
\hline Gynäkologen & 94,12 & 5,971 & 1,024 & 94,12 & 34 \\
\hline Pädiater & 96,60 & 3,863 & 0,576 & 100,00 & 45 \\
\hline Anästhesisten & 93,44 & 5,081 & 0,997 & 94,12 & 26 \\
\hline Hebammen & 87,88 & 10,496 & 1,827 & 88,24 & 33 \\
\hline $\begin{array}{l}\text { Kinderkranken- } \\
\text { schwestern }\end{array}$ & 87,50 & 10,852 & 1,918 & 88,24 & 32 \\
\hline Insgesamt & 92,21 & 8,431 & 0,647 & 94,12 & 170 \\
\hline
\end{tabular}

Tabelle 6: Vergleich der Ergebnisse der unterschiedlichen Berufsgruppen direkt nach dem Kurs 


\subsubsection{Vergleich der Ergebnisse zwischen den Berufsgruppen nach sechs Monaten ( $\mathrm{t} 3$ )}

Nach sechs Monaten war zwischen den verschiedenen Berufsgruppen weiterhin ein signifikanter Wissensunterschied erkennbar $(p=0,001)$. Die Unterschiede im Wissensstand entsprachen den in den Voruntersuchungen erkennbaren Unterschieden, wobei die Gruppe der Hebammen weniger Wissensverlust aufwies, als die Gruppe der Kinderkrankenschwestern. Die Gruppe der Kinderkrankenschwestern hatte nach sechs Monaten fast wieder das Ausgangsniveau im Prozentsatz der richtig beantworteten Fragen erreicht (siehe Tabelle 7).

\begin{tabular}{|c|c|c|c|c|c|}
\hline Berufsgruppe & $\begin{array}{l}\text { Mittelwert } \\
(\%)\end{array}$ & $\begin{array}{l}\text { Standard- } \\
\text { abweichung } \\
(\%)\end{array}$ & $\begin{array}{l}\text { Standardfehler } \\
\text { des Mittelwertes } \\
(\%)\end{array}$ & $\begin{array}{l}\text { Median } \\
(\%)\end{array}$ & $\begin{array}{l}\mathbf{N} \\
\text { (Teilnehmerz } \\
\text { ahl) }\end{array}$ \\
\hline Gynäkologen & 88,24 & 14,00 & 5,293 & 88,24 & 7 \\
\hline Pädiater & 92,94 & 5,070 & 1,309 & 94,12 & 15 \\
\hline Anästhesisten & 85,29 & 8,108 & 3,310 & 88,24 & 6 \\
\hline Hebammen & 85,47 & 7,814 & 1,895 & 82,35 & 17 \\
\hline $\begin{array}{l}\text { Kinderkranken- } \\
\text { schwestern }\end{array}$ & 77,21 & 7,332 & 2,592 & 76,47 & 8 \\
\hline Insgesamt & 86,68 & 9,383 & 1,289 & 88,24 & 53 \\
\hline
\end{tabular}

Tabelle 7: Vergleich der Ergebnisse der unterschiedlichen Berufsgruppen nach sechs Monaten 


\subsection{Auswertung der subjektiven Beurteilungsbögen}

Die Evaluation der subjektiven Bewertungsbögen sollte subjektiv empfundene Schwächen der Schulung aufzeigen und damit Hinweise auf Probleme in Organisation und Durchführung des Kurses geben. Gleichzeitig war die Möglichkeit gegeben, konkrete Verbesserungsvorschläge zu machen. Der Aufbau eines Beurteilungsbogens kann im Anhang nachgelesen werden.

An der Befragung nach dem Kurs hinsichtlich der subjektiven Bewertung der Schulung nahmen 93\% aller Teilnehmer teil. 79\% der Fragebögen enthielten zusätzlich handschriftliche Kommentare. Es wurden 172 Beurteilungsbögen aus acht Kursen ausgewertet.

Die Teilnehmer sollten den Kurs anhand von klar ausformulierten Fragen mit Punkten von ++ (für sehr gut/stimmt genau) bis -- (für sehr schlecht/stimmt überhaupt nicht) beurteilen. Zur besseren Auswertbarkeit wurde das Ergebnis dann in Punkte von fünf bis eins übertragen.

\subsubsection{Gesamtüberblick über die Kursbewertung}

Zur besseren Übersicht über die Bewertung wird hier eine Tabelle (Tabelle 8) mit den Mittelwerten der von 172 Teilnehmern vergebenen Beurteilungspunkte vorgelegt.

Die beste Beurteilung entsprechend der Auswertung der Gesamtzahl der Meinungen erhielten der praktische Nutzen des Kurses (4,92 Punkte) und die Kompetenz der Referenten (4,91 Punkte). Die Standardabweichungen waren bei beiden Fragestellungen ausgesprochen klein (0,26 und 0,31). Das bedeutet, dass sich die Kursteilnehmer hinsichtlich dieser Beurteilung einig waren.

Auffallend schlecht schnitt die Frage nach der Kursdauer ab (4,39 Punkte). Hier konnte eine hohe Standardabweichung berechnet werden, die Kursteilnehmer waren also in ihrer Punktevergabe recht unterschiedlich.

In der Zusammenfassung lässt sich sagen, dass der Kurs von den Teilnehmern gut angenommen und generell sehr positiv beurteilt wurde. 


\begin{tabular}{|l|l|l|l|}
\hline Fragestellung & Spannweite & $\begin{array}{l}\text { Standard- } \\
\text { Abweichung }\end{array}$ & Mittelwert \\
\hline $\begin{array}{l}\text { Bedeutung: Wie schätzen Sie den } \\
\text { praktischen Nutzen des Kurses für Ihre } \\
\text { Arbeit ein? }\end{array}$ & $4-5$ & 0,26 & 4,92 \\
\hline $\begin{array}{l}\text { Qualität: Wie beurteilen Sie den } \\
\text { Informationsgehalt des Seminars? }\end{array}$ & $4-5$ & 0,40 & 4,80 \\
\hline $\begin{array}{l}\text { Effizienz: Wurden Sie angeregt, einige } \\
\text { Aspekte Ihrer medizinischen Tätigkeit zu } \\
\text { überdenken oder zu ändern? }\end{array}$ & $3-5$ & 0,57 & 4,60 \\
\hline $\begin{array}{l}\text { Referenten 1: Wie beurteilen Sie die } \\
\text { Fachkompetenz der Referenten } \\
\text { insgesamt? }\end{array}$ & $3-5$ & 0,31 & 4,91 \\
\hline $\begin{array}{l}\text { Referenten 2: Wie verständlich war die } \\
\text { Darstellung? }\end{array}$ & $2-5$ & 0,41 & 4,84 \\
\hline $\begin{array}{l}\text { Organisation: War die Dauer der } \\
\text { Veranstaltung angemessen? }\end{array}$ & $1-5$ & 0,69 & 4,39 \\
\hline $\begin{array}{l}\text { Fazit 1: Hat das Seminar bezüglich } \\
\text { Zielsetzung und Inhalten Ihre } \\
\text { Erwartungen erfüllt? }\end{array}$ & $3-5$ & 0,38 & 4,84 \\
\hline $\begin{array}{l}\text { Fazit 2: Wie beurteilen Sie das Seminar } \\
\text { insgesamt? }\end{array}$ & $3-5$ & 0,37 & 4,85 \\
\hline
\end{tabular}

Tabelle 8: Beurteilung des Kurses durch alle 172 Teilnehmer

\subsubsection{Einzelauswertung der gestellten Fragen}

Bedeutung: Wie schätzen Sie den praktischen Nutzen des Kurses für Ihre Arbeit ein?

Der praktische Nutzen des Kurses wird von allen Teilnehmergruppen als "sehr hoch“ (Durchschnitt 4,92 Punkte) eingestuft. Dabei ziehen Gynäkologen, Pädiater und Kinderkrankenschwestern subjektiv den größten Nutzen aus diesem Kurs, Anästhesisten sehen am wenigsten Nutzen in ihrem alltäglichen Arbeitsfeld (siehe Tabelle 9).

\begin{tabular}{|l|c|l|l|l|l|c|}
\hline Berufsgruppe & $\begin{array}{l}\text { Gynäko- } \\
\text { logen }\end{array}$ & Pädiater & $\begin{array}{l}\text { Anästhe- } \\
\text { sisten }\end{array}$ & $\begin{array}{l}\text { Heb- } \\
\text { ammen }\end{array}$ & $\begin{array}{l}\text { Kinder- } \\
\text { kranken- } \\
\text { schwestern }\end{array}$ & Sonstige \\
\hline Mittelwert & 5,00 & 4,94 & 4,81 & 4,90 & 4,94 & 4,93 \\
\hline Spannweite & $5-5$ & $4-5$ & $4-5$ & $4-5$ & $4-5$ & $4-5$ \\
\hline Standardabw. & 0,00 & 0,24 & 0,39 & 0,30 & 0,25 & 0,26 \\
\hline
\end{tabular}

Tabelle 9: Beurteilung des praktischen Nutzens des Kurses 


\section{Qualität: Wie beurteilen Sie den Informationsgehalt des Seminars?}

Der Informationsgehalt des Kurses wird ebenfalls als "sehr hoch“ eingeschätzt (Durchschnitt 4,80 Punkte).

Am stärksten scheinen Gynäkologen, Hebammen und Kinderkrankenschwestern von den theoretischen Vorträgen zu profitieren (siehe Tabelle 10).

\begin{tabular}{|l|l|c|c|l|l|c|}
\hline Berufsgruppe & $\begin{array}{l}\text { Gynäko- } \\
\text { logen }\end{array}$ & Pädiater & $\begin{array}{l}\text { Anästhe- } \\
\text { sisten }\end{array}$ & $\begin{array}{l}\text { Heb- } \\
\text { ammen }\end{array}$ & $\begin{array}{l}\text { Kinder- } \\
\text { kranken- } \\
\text { schwestern }\end{array}$ & Sonstige \\
\hline Mittelwert & 4,89 & 4,68 & 4,77 & 4,81 & 4,81 & 4,86 \\
\hline Spannweite & $4-5$ & $4-5$ & $4-5$ & $4-5$ & $4-5$ & $4-5$ \\
\hline Standardabw. & 0,31 & 0,47 & 0,42 & 0,40 & 0,40 & 0,26 \\
\hline
\end{tabular}

Tabelle 10: Beurteilung des Informationsgehaltes des Seminars

Effizienz: Wurden Sie angeregt, einige Aspekte Ihrer medizinischen Tätigkeit zu überdenken oder zu ändern?

Fast alle Teilnehmer gaben an, Teilaspekte ihrer medizinischen Tätigkeit ändern oder überdenken zu wollen (Durchschnitt: 4,60 Punkte).

Offensichtlich profitieren alle Berufsgruppen nahezu gleichermaßen von diesem Kurs (siehe Tabelle 11).

\begin{tabular}{|l|c|c|c|c|c|c|}
\hline Berufsgruppe & $\begin{array}{l}\text { Gynäko- } \\
\text { logen }\end{array}$ & Pädiater & $\begin{array}{l}\text { Anästhe- } \\
\text { sisten }\end{array}$ & $\begin{array}{l}\text { Heb- } \\
\text { ammen }\end{array}$ & $\begin{array}{l}\text { Kinder- } \\
\text { kranken- } \\
\text { schwestern }\end{array}$ & Sonstige \\
\hline Mittelwert & 4,72 & 4,56 & 4,46 & 4,61 & 4,61 & 4,57 \\
\hline Spannweite & $2-5$ & $3-5$ & $3-5$ & $3-5$ & $4-5$ & $3-5$ \\
\hline Standardabw. & 0,61 & 0,55 & 0,57 & 0,55 & 0,49 & 0,62 \\
\hline
\end{tabular}

Tabelle 11: Beurteilung der Effizienz des Seminars

Referenten 1: Wie beurteilen Sie die Fachkompetenz der Referenten insgesamt?

Die Fachkompetenz der Referenten wurde von allen Teilnehmern als "sehr hoch“ eingeschätzt (Durchschnitt: 4,91 Punkte).

Die Referenten dieses Kurses sind ausschließlich Pädiater oder Kinderkrankenschwestern. Diese wurden insbesondere von ihren pädiatrischen und pflegerischen Kollegen als sehr kompetent eingestuft (siehe Tabelle 12). 


\begin{tabular}{|l|c|c|c|c|c|c|}
\hline Berufsgruppe & $\begin{array}{l}\text { Gynäkolo } \\
\text { gen }\end{array}$ & Pädiater & $\begin{array}{l}\text { Anästhesi } \\
\text { sten }\end{array}$ & $\begin{array}{l}\text { Hebamme } \\
\mathbf{n}\end{array}$ & $\begin{array}{l}\text { Kinder- } \\
\text { Krankensch } \\
\text { western }\end{array}$ & Sonstige \\
\hline Mittelwert & 4,94 & 4,88 & 4,81 & 4,94 & 4,97 & 4,86 \\
\hline Spannweite & $4-5$ & $4-5$ & $3-5$ & $4-5$ & $4-5$ & $4-5$ \\
\hline Standardabw. & 0,23 & 0,32 & 0,48 & 0,25 & 0,18 & 0,35 \\
\hline
\end{tabular}

Tabelle 12: Beurteilung der Fachkompetenz der Referenten

\section{Referenten 2: Wie verständlich war die Darstellung?}

Für alle Teilnehmer scheinen die Kurse sehr gut verständlich (Durchschnitt 4,84 Punkte), Hebammen werden weniger von der Darstellungsweise angesprochen, als Kinderkrankenschwestern und Gynäkologen (siehe Tabelle 13).

\begin{tabular}{|l|c|c|c|c|c|c|}
\hline Berufsgruppe & $\begin{array}{l}\text { Gynäkolo } \\
\text { gen }\end{array}$ & Pädiater & $\begin{array}{l}\text { Anästhesi } \\
\text { sten }\end{array}$ & $\begin{array}{l}\text { Hebamme } \\
\text { n }\end{array}$ & $\begin{array}{l}\text { Kinder- } \\
\text { Krankensch } \\
\text { western }\end{array}$ & Sonstige \\
\hline Mittelwert & 4,94 & 4,82 & 4,81 & 4,68 & 4,94 & 4,56 \\
\hline Spannweite & $4-5$ & $4-5$ & $2-5$ & $4-5$ & $4-5$ & $4-5$ \\
\hline Standardabw. & 0,23 & 0,38 & 0,62 & 0,47 & 0,25 & 0,41 \\
\hline
\end{tabular}

Tabelle 13: Beurteilung der Verständlichkeit der Darstellung

\section{Organisation: War die Dauer der Veranstaltung angemessen?}

Die Dauer des Kurses wurde von allen Gruppen als angemessen bewertet. Bei dieser Frage wurde trotzdem eine auffallend schlechte Punktzahl vergeben (Durchschnitt 4,39). Von Seiten der Anästhesisten wurde die Kursdauer als besonders schlecht beurteilt (siehe Tabelle 14). Der Hintergrund dieser niedrigen Bewertungen wurde durch die Auswertung der handschriftlichen Bemerkungen erkennbar (siehe Abschnitt 5.3.4).

\begin{tabular}{|l|c|l|c|c|c|c|}
\hline Berufsgruppe & $\begin{array}{l}\text { Gynäkolo } \\
\text { gen }\end{array}$ & Pädiater & $\begin{array}{l}\text { Anästhesi } \\
\text { sten }\end{array}$ & $\begin{array}{l}\text { Hebamme } \\
\mathbf{n}\end{array}$ & $\begin{array}{l}\text { Kinder- } \\
\text { Krankensch } \\
\text { western }\end{array}$ & Sonstige \\
\hline Mittelwert & 4,44 & 4,44 & 4,12 & 4,45 & 4,39 & 4,50 \\
\hline Spannweite & $1-5$ & $3-5$ & $3-5$ & $3-5$ & $1-5$ & $3-5$ \\
\hline Standardabw. & 0,76 & 0,55 & 0,64 & 0,61 & 0,83 & 0,63 \\
\hline
\end{tabular}

Tabelle 14: Beurteilung der Kursdauer 


\section{Fazit 1: Hat das Seminar bezüglich Zielsetzung und Inhalten Ihre Erwartungen erfüllt?}

Die Zielsetzungen des Seminars sind in den Augen aller Berufsgruppen erfült worden (Durchschnitt 4,84). Besonders zufrieden mit der Schulung waren Kinderkrankenschwestern und Gynäkologen. Nicht vollständig angesprochen fühlten sich die Anästhesisten und Pädiater (siehe Tabelle 15).

\begin{tabular}{|l|c|l|l|l|l|c|}
\hline Berufsgruppe & $\begin{array}{l}\text { Gynäkolo } \\
\text { gen }\end{array}$ & Pädiater & $\begin{array}{l}\text { Anästhesi } \\
\text { sten }\end{array}$ & $\begin{array}{l}\text { Hebamme } \\
\mathbf{n}\end{array}$ & $\begin{array}{l}\text { Kinder- } \\
\text { Krankensch } \\
\text { western }\end{array}$ & Sonstige \\
\hline Mittelwert & 4,92 & 4,74 & 4,65 & 4,87 & 4,97 & 4,86 \\
\hline Spannweite & $4-5$ & $4-5$ & $3-5$ & $4-5$ & $4-5$ & $4-5$ \\
\hline Standardabw. & 0,28 & 0,44 & 0,55 & 0,34 & 0,18 & 0,35 \\
\hline
\end{tabular}

Tabelle 15: Beurteilung, ob die subjektiven Erwartungen erfüllt wurden.

\section{Fazit 2: Wie beurteilen Sie das Seminar insgesamt?}

Entsprechend den vorhergegangenen guten Beurteilungen des Kurses fält auch die subjektive Gesamtbeurteilung sehr gut aus (Durchschnitt 4,85 Punkte). Die relativ schlechte Gesamtbeurteilung durch Anästhesisten und Pädiater (siehe Tabelle 16) ist am ehesten durch die spezifischen Vorstellungen und Verbesserungsvorschläge dieser Berufsgruppen begründet. Auf diese Punkte wird im Abschnitt 5.3.4 genauer eingegangen.

\begin{tabular}{|l|c|c|c|c|c|c|}
\hline Berufsgruppe & $\begin{array}{l}\text { Gynäkolo } \\
\text { gen }\end{array}$ & Pädiater & $\begin{array}{l}\text { Anästhesi } \\
\text { sten }\end{array}$ & $\begin{array}{l}\text { Hebamme } \\
\mathbf{n}\end{array}$ & $\begin{array}{l}\text { Kinder- } \\
\text { Krankensch } \\
\text { western }\end{array}$ & Sonstige \\
\hline Mittelwert & 4,92 & 4,82 & 4,65 & 4,90 & 4,91 & 4,79 \\
\hline Spannweite & $4-5$ & $4-5$ & $3-5$ & $4-5$ & $4-5$ & $4-5$ \\
\hline Standardabw. & 0,28 & 0,38 & 0,55 & 0,30 & 0,25 & 0,41 \\
\hline
\end{tabular}

Tabelle 16: Gesamtbeurteilung des Seminars

\subsubsection{Auswertung des freien Beurteilungsbogens}

In diesem Abschnitt soll im Einzelnen auf die handschriftlich geäußerte Kritik der Teilnehmer eingegangen werden. Von den 172 befragten Teilnehmern nahmen 89\% die Möglichkeit zu freier Meinungsäußerung wahr. Viele positive und negative Kritikpunkte wurden von mehreren Teilnehmern und Teilnehmerinnen (im Folgenden 
Teilnehmer), unabhängig von der Berufsgruppenzugehörigkeit geäußert, einige Kritikpunkte schienen eher berufsspezifischer zu sein.

\section{Kursstruktur und Kursorganisation}

Die gute Strukturierung des Kurses, die straffe Organisation wurde von elf Teilnehmern aus allen Berufsgruppen positiv hervorgehoben.

Neun Teilnehmer empfanden den Kurs als zu lang. Vier von diesen schlugen vor einen zweitägigen Kurs anzubieten. Drei Teilnehmer baten um mehr, drei Teilnehmer baten um weniger Pausen.

Zwölf Teilnehmer empfanden die Aufteilung der Teilnehmer in kleine Gruppen von vier bis fünf Teilnehmern pro Gruppe bei den praktischen Übungen als positiv, wohingegen fünf Teilnehmer um noch kleinere Gruppen baten.

\section{Theoretischer Teil/Vorträge}

In allen Berufsgruppen wurden die Vorträge als prägnant, abwechslungsreich und mit Betonung auf das Wesentliche beschrieben, so dass die theoretischen Grundlagen gut aufzunehmen seien (sechs Meinungen). Auch die Überprüfung des Wissens durch die Dozenten wurde als positiv wahrgenommen.

16 Teilnehmer betonten die hohe Kompetenz der Referenten. Durch die verschiedenen Referenten würden die Themen aus unterschiedlichen Blickwinkeln erklärt. Neun Teilnehmer aus jeglichen Berufsgruppen schrieben, dass Theorie und Praxis gut aufeinander abgestimmt seien.

Drei Teilnehmer baten um ein ausführliches Skript (Anm.: Es wurde im Kurs nur ein Handzettel mit dem Reanimationsschema für Neugeborene ausgeteilt).

Fünf Personen hoben hervor, dass immer Zeit für Rückfragen gegeben war und jegliche Frage kompetent und ruhig beantwortet wurde, ein Teilnehmer wünschte sich mehr Zeit für Fragen.

Auf der Seite der negativen Kritik wurde von Pädiatern, Anästhesisten und Gynäkologen mehr Theorie gefordert, dabei insbesondere eine komplexere Darstellung der medikamentösen Therapie, die über Adrenalin, Volumen und 
Glucose hinausginge, sowie die Besprechung des weiteren Vorgehens bei protrahiertem Reanimationsverlauf (vier Meinungen).

Zwei Hebammen dagegen baten um weniger Theorie, bzw. eine einfachere Darstellung, insbesondere weniger Betonung auf die medikamentöse Therapie.

\section{Praktische Übungen}

Von 92 Teilnehmern wurde der große Schwerpunkt auf den praktischen Übungen als sehr positiv hervorgehoben, 14 Teilnehmer empfanden die Szenarien als wirklichkeitsgetreu und praxisrelevant.

Die häufige Wiederholung des Reanimationsschemas wurde als förderlich empfunden (15 Meinungen). Zudem habe es eine gute Supervision gegeben (drei Meinungen), bei der auch auf individuelle Probleme eingegangen worden sei. Jeder Teilnehmer habe die Möglichkeit bekommen, mehrfach aktiv zu werden, und sich dabei im interdisziplinären Team zu engagieren.

Vereinzelt wurde um mehr oder weniger praktische Übungen gebeten (jeweils zwei Meinungen).

Pädiater und Anästhesisten wünschen sich mehr Intubationsmöglichkeiten und mehr Möglichkeiten, Gefäßzugänge zu üben (drei Meinungen).

Eine Hebamme bat darum, Hausgeburten nicht so negativ darzustellen, da nicht jede Hausgeburt zu einer Reanimationssituation führe.

\section{Fragebogen}

16 Teilnehmer empfanden die Fragen des Fragebogens als zu pauschal formuliert und missverständlich. Elf Teilnehmer hätten sich eine direkte Besprechung der Lösungen oder einen Lösungsbogen gewünscht.

\section{Atmosphäre}

15 Teilnehmer hoben die nette, lockere Atmosphäre, die herzlichen und engagierten Dozenten und das konstruktive Feedback hervor. 


\section{Persönlicher Gewinn}

Elf Teilnehmer aus allen Berufsgruppen gaben an, nach dem Kurs weniger Angst vor einem Notfall zu haben und an Selbstbewusstsein dazu gewonnen zu haben: „ich habe jetzt weniger Angst“, „ich habe zum ersten Mal das Gefühl, im Notfall ein richtiges Schema im Kopf zu haben“. 


\section{Diskussion}

\subsection{Vorgehensweisen bei der Evaluation von Reanimationskursen}

Neugeborenenreanimationskurse haben zum Ziel, medizinisches Personal in die Lage zu versetzen, Neugeborene gerade in den ersten kritischen Minuten optimal zu versorgen (Patel et al. 2001).

Die in Europa angebotenen Schulungen in Neugeborenenreanimation werden in einigen Krankenhäusern selbstständig als interne Kurse entwickelt und angeboten, teilweise werden NLS-Kurse (auf Grundlage der Richtlinien des ERC: Baskett et al. 2005, Handbuch durch S. Richmond 2008) mehrheitlich aber NRP-Kurse (auf Grundlage der Richtlinien der AHA und der AAP: AHA 2005, Handbuch durch J. Kattwinkel 2006) angeboten.

Die Kurse des ERC und der AHA sind in inrem Aufbau ähnlich, aber bisher noch nicht vollkommen einheitlich (Raupp and McCutcheon 2007). Im Rahmen des International Liaison Committee on Resuscitation (ILCOR, Gründung 1992), ein Verbund aus sechs großen, internationalen Reanimationsgesellschaften (unter anderem auch die AHA und der ERC), wird versucht, Kursstruktur und Kursinhalte der Reanimationskurse anzupassen (Chamberlain et al. 2005). Teilnehmer, die sowohl den NRP als auch den NLS-Kurs absolviert hatten, empfanden trotz der bekannten Differenzen in den Kursinhalten (Raupp and McCutcheon 2007) keine Irritation hinsichtlich ihres zukünftigen Vorgehens (Pederzini et al. 2008). Dieselben Teilnehmer zeigten sich hinsichtlich der praktischen und theoretischen Wissensvermittlung in beiden Kursen zufrieden. Dies deutet darauf hin, dass beide Kurssysteme in Bezug auf Inhalt und Effektivität vergleichbar sind.

Die Effektivität von Reanimationskursen kann auf zweierlei Weise überprüft werden: Zum einen, indem Morbidität und Mortalität der Kinder vor und nach einem Reanimationstraining überprüft werden (Patel et al. 2001, Duran et al. 2008a). Zum anderen, indem der Lernerfolg und die Zufriedenheit der Teilnehmer anhand von Fragebögen evaluiert werden (Dunn et al. 1992, Levitt et al. 1996, Trevisanuto et al. 2005, Duran et al. 2008b). 
Die Auswertung von Morbidität und Mortalität der Kinder nach Reanimation ist eine sehr aussagekräftige Methode, um Reanimationskurse in ihrer Effektivität und ihrer praktischen Anwendung im Alltag zu überprüfen. Dazu ist es notwendig, die Geburtshelfer einer Region oder zumindest eines Krankenhauses nahezu vollständig auf der Grundlage eines einheitlichen Fortbildungssystems zu trainieren, und diesen regelmäßige Auffrischungskurse anzubieten, um den Wissensstand des Personals stetig auf hohem Niveau zu erhalten. Auf diese Weise wird eine Referenzgruppe im Vergleich zu den vorhergehenden Jahren oder zu anderen Regionen/Krankenhäusern gebildet. In den USA beispielsweise werden seit 1987 flächendeckend Neugeborenen-Reanimationskurse im Rahmen des Neonatal Resuscitation Program angeboten und genutzt (Patel et al. 2001, Raupp and McCutcheon 2007). In den Untersuchungen bezüglich des Outcome der Kinder, die von trainiertem Personal behandelt wurden, konnte ein deutlicher Gewinn hinsichtlich Mortalität und Morbidität der Kinder nachgewiesen werden (Patel et al. 2001, Duran et al. 2008a, Carcillo et al. 2009).

Eine solche Studie ist in Deutschland derzeit noch nicht durchführbar, da bisher eine flächendeckende Versorgung der deutschen Krankenhäuser mit entsprechenden Fortbildungsangeboten nicht vorhanden ist. Aufgrund von finanziellen und logistischen Problemen kann zum derzeitigen Zeitpunkt keine vollständige Fortbildung des Personals einzelner Kliniken gewährleistet werden. Eine Ausnahme könnte das Städtische Klinikum Friedrichstadt in Dresden darstellen, das den NRPKurs für eigenes und externes Personal anbietet (Lorenz et al. 2007). Es gibt ansonsten in den kleineren Krankenhäusern ohne Perinatalzentrum nie die Konstellation, dass nahezu das gesamte Reanimationsteam in neonataler Reanimation ausgebildet ist. Somit ist eine aussagekräftige Datenlage hinsichtlich neonataler Mortalität und Morbidität vor und nach Einführung eines Reanimationskurses in Deutschland nicht gegeben.

In Deutschland, wie auch in anderen Ländern Europas, sind NeugeborenenReanimationskurse noch nicht flächendeckend verbreitet. In einer Umfrage im Jahr 2002/2003 durch K. Foster in New South Wales (United Kingdom, im Folgenden UK) gaben ein Drittel der befragten Geburtshelfer aus peripheren Krankenhäusern an, noch nie oder vor mehr als zwei Jahren an einem Reanimationskurs teilgenommen zu haben. In den ländlichen Regionen waren nur $40 \%$ der Geburtshelfer zufrieden 
mit ihren Reanimations-Kenntnissen, 75-90\% aller Geburtshelfer sahen Verbesserungspotentiale hinsichtlich des Angebotes an Reanimationskursen (Foster et al. 2006).

In Großbritannien wurde eine entsprechende Studie über den Effekt von Reanimationskursen durchgeführt, indem das Personal einer Universitätsklinik (Northern and Yorkshire Hospital, UK) seit 1995 vollständig und regelmäßig geschult wurde. Es konnte eine Verminderung des Medikamentengebrauches und eine Verminderung von Hypothermie bei Risikoneugeborenen nach Einführung eines praxisbetonten Reanimationskurses im Jahr 1995 und der Etablierung des NLSKurses des ERC im Jahr 2002 im Vergleich zu den Vorjahren nachgewiesen werden (Singh et al. 2006).

Mit Blick auf die Studien in Großbritannien und den USA ist daher eine Überprüfung der Effektivität von Reanimationsangeboten in Deutschland angezeigt. Zumal es derzeit nicht absehbar ist, dass sich die vorstehend ausgeführte Situation für Deutschland innerhalb der nächsten Jahre ändert, ist eine Untersuchung von Mortalität und Morbidität von Kindern vor und nach Reanimationskursen, die zu belastbaren Ergebnissen führt, derzeit nicht möglich. Somit stellt die direkte Evaluation des Wissenstandes oder der Fertigkeiten der Teilnehmer derzeit die einzige Möglichkeit dar, die Effektivität von Reanimationskursen in Deutschland zu überprüfen. 


\subsection{Planung der Evaluation eines Reanimationskurses}

Eine Überprüfung der Effektivität von Reanimationskursen mittels einer direkten Evaluation derselben bedarf zur Erzielung von belastbaren Ergebnissen einer umfangreichen Vorbereitung. Ein entsprechender Fragebogen muss derart konzipiert sein, dass aus den Antworten belastbare Aussagen zu dem Nutzen des Reanimationskurses (und des dahinter stehenden Konzeptes) für die Probanden (d.h. für die Kursteilnehmer) in Hinblick auf die spätere Reanimationstätigkeit gewonnen werden können. Hierzu sind zunächst die Faktoren herauszuarbeiten, die einen Reanimationsablauf qualitativ beeinflussen. Diesen Faktoren sind einzelne Kenntnisse und Fähigkeiten zuzuordnen, deren Vorliegen, respektive Beherrschung zu einem guten Reanimationsablauf führen. Auf dieser Grundlage sind einzelne Fragen zu entwickeln bzw. auszuwählen, aufgrund derer sich über den jeweiligen Stand der Fähigkeiten und Kenntnisse belastbare Aussagen treffen lassen.

Diese vorstehend genannten Faktoren und die daraus abzuleitenden Kenntnisse und Fähigkeiten stellen zudem auch den Ausgangspunkt für die Definition der Ziele und Lerninhalte der entsprechenden Schulungen dar. Somit ist im Rahmen der Evaluation auch zu prüfen, ob und inwieweit die definierten Ziele und Lerninhalte der Reanimationskurse erreicht werden.

Gerade in Hinblick auf die für einen guten Reanimationsablauf wichtigen Faktoren und die dem Kurskonzept zu Grunde liegenden Lernziele und Lerninhalte ist es nach Auswertung der Ergebnisse sinnvoll, Verbesserungsvorschläge für den Kursablauf auszuarbeiten. Hierzu ist auch ein zeitlicher Rahmen zu erarbeiten, innerhalb dessen ein Reanimationskurs wiederholt werden sollte, um einen kontinuierlich hohen Reanimationsstandard aufrechtzuerhalten.

\subsubsection{Faktoren für einen qualitativ guten Reanimationsablauf}

Hinsichtlich der Ermittlung der Faktoren für einen qualitativ guten Reanimationsverlauf kann man zunächst auf eine Studie von Verplancke et al. (2008), welche eine Studie hinsichtlich Basic Life Support am Erwachsenen durchgeführt haben, zurückgreifen. Als die für eine qualitativ gute Reanimation maßgeblichen Faktoren wurden in der genannten Studie die nachstehend aufgeführten genannt: 
- Gute Kenntnisse (kurz zurückliegender Reanimationskurs) bzw. Übung (in Form einer kurz zurückliegenden Reanimation)

- Hohes Selbstbewusstsein

- Maskulines Geschlecht.

Bei der Bewertung dieser Faktoren sind auch Untersuchungen der „Joint Commission on Accreditation of Healthcare Organisations" in den USA (2004) zu beachten, wonach unzureichende Kommunikation (72\%) und mangelnde Ausbildung (47\%) die Hauptgründe für medizinisches Fehlverhalten sind. Bei diesen Untersuchungen werden Hierarchieprobleme und Einschüchterung als ursächlich für Kommunikationsprobleme angegeben. Somit ist eine respektvolle, aber selbstbewusste und konstruktive Kommunikation zwischen den Geburtshelfern ausschlaggebend für die Vermeidung von Fehlern und damit auch ein Faktor für die hohe Qualität der medizinischen Maßnahmen und den Erfolg der Reanimation. Zumal Fehler von Einzelnen letztendlich zu einem schlechten Outcome des Kindes führen können (Simpson et al. 2006, Jukkala and Henly 2009), ist daher insgesamt eine zielgerichtete Zusammenarbeit aller bei einer Reanimation involvierten Personen zur Vermeidung von Fehlern wichtig.

Zusammenfassend lassen sich die vorstehend genannten Faktoren in objektive und subjektive Faktoren unterteilen.

Zu den objektiven Faktoren gehören vor allem die theoretischen und praktischen Kenntnisse und Fähigkeiten des Reanimierenden. Die Person, die die Reanimation durchführt und/oder leitet, muss über ein umfangreiches, auch in Stresssituationen abrufbares Wissen über den Reanimationsablauf verfügen und dieses Wissen adäquat und konstruktiv einsetzen können. Dieses Wissen lässt sich über die Fragebögen im Rahmen einer Evaluation abprüfen.

Zumal sich allein anhand theoretischer Ergebnisse keine belastbare Aussage über die für eine Reanimation erforderlichen praktischen Fähigkeiten treffen lässt (siehe auch Rogers et al. 2001), sind praktische Übung und Überprüfung zur finalen Beurteilung der Effektivität unerlässlich.

Zu den objektiven Faktoren zählt nach der Studie von Verplancke et al. (2008) auch das Geschlecht desjenigen, der die Reanimation durchführt. Die Studienleiter vermuteten einen Zusammenhang mit der größeren Kraft der Männer. Dadurch seien 
tiefere und effektivere Kompressionen bei der Herz-Druck-Massage beim erwachsenen Patienten möglich, was in der Studie die Qualität der Reanimation beeinflusst hätte. Für die vorliegende Arbeit ist jedoch zu bedenken, dass das KraftWiderstands-Verhältnis in der Neugeborenenreanimation ein anderes als bei der Reanimation von Erwachsenen ist. Folglich kommen die körperlichen Unterschiede zwischen Männern und Frauen in der Neugeborenenreanimation nur bedingt zum Tragen. Insofern ist das maskuline Geschlecht für die in dieser Arbeit zu untersuchende Reanimation von Neugeborenen deutlich weniger bedeutsam. Zumal dieser Faktor durch einen Reanimationskurs nicht zu beeinflussen ist, wird er in den folgenden Überlegungen nicht weiter beachtet.

Als subjektive Faktoren für einen qualitativ guten Reanimationsablauf sind die Bewertung der eigenen Kenntnisse und das $\mathrm{Ma} B$ an Selbstvertrauen von Bedeutung. Dies wird anhand der vorstehend genannten Hierarchieprobleme deutlich. Dabei hängt die Qualität einer Reanimation nicht vom hierarchischen Status der reanimierenden Person ab (Chan and Hey 2006). So kann eine Reanimation sowohl von einer Hebamme, einer Fach-Krankenschwester oder von einem Arzt geleitet werden.

In dem „Janeway Childrens Health and Rehabilitation Centre“ wurde ein interdisziplinäres Notfall-Team gegründet, das präventiv zu Risiko- und Hochrisikogeburten geholt wurde. Bei einem Viertel der Einsätze bei Risikogeburten bestand das Team aus speziell geschulten Pflegekräften (Aziz et al. 2005). Weder in dieser noch in anderen Studien (Aubrey and Yoxall 2001; Chan and Hey 2006) konnte eine Verschlechterung des Outcomes der Kinder nachgewiesen werden, wenn die Reanimation durch ausgebildete Pflegekräfte statt durch Ärzte durchgeführt wurde. Hierarchische Probleme können jedoch dazu führen, dass die für die vorliegende Reanimationssituation am besten ausgebildete Person ihre Fähigkeiten nicht voll ausschöpfen kann. Dabei ist es gerade bei unterschiedlichen hierarchischen Stufen für eine gelungene Reanimation wichtig, dass sich alle an einer Reanimation teilnehmenden Personen hinsichtlich der zu treffenden Maßnahmen sicher sind. Das bedeutet, dass diese Personen diesbezüglich über ein hohes Selbstbewusstsein verfügen sollten und ihr Wissen respektvoll, aber selbstbewusst kommunizieren. Ob die Teilnahme an einem NLS-Kurs einen subjektiv 
empfundenen Nutzen für die Teilnehmer hatte und deren Selbstbewusstsein gestärkt werden konnte, kann anhand eines Beurteilungsbogens ausgewertet werden.

Aus den oben genannten Punkten ergibt sich, dass ein Reanimationskurs anhand der zusammengefassten Ergebnisse aus einem Fragebogen, der das theoretische Wissen überprüft, einem praktischen Test, der die erworbenen praktischen Fähigkeiten testet, und einem Beurteilungsbogen, der das subjektive Empfinden hinsichtlich des persönlichen Gewinns abfragt, umfassend beurteilt werden kann (siehe auch Rogers et al. 2001).

Einschränkend muss hinzugefügt werden, dass hiermit noch keine Aussage über die praktische Durchführung der Kenntnisse im Berufsalltag getroffen werden kann. Duran et al. zeigen aber auf, dass nach Einführung einer Beschulung des Personals mittels eines NRP-Kurses, der zu einer deutlichen Verbesserung des Wissens der Teilnehmer führte (Duran et al. 2008b), in diesem Krankenhaus auch eine Senkung der kindlichen Mortalität nachweisbar war (Duran et al. 2008a). Aus diesem Grund wird auch für die vorliegende Untersuchung davon ausgegangen, dass ein nachweislich effektiver Kurs im klinischen Alltag einen Benefit für die kleinen Patienten bringt. Der Beweis hierfür steht noch aus und sollte, sobald möglich, in Folgestudien erbracht werden.

\subsubsection{Ziele und Lerninhalte eines Reanimationskurses}

Aufgrund des Erfordernisses, auch in Stresssituationen Wissen adäquat und konstruktiv einsetzen zu können, sollte der Teilnehmer eines Reanimationskurses ein schnell und klar abrufbares Handlungsschema sowohl im theoretischen Hintergrund als auch hinsichtlich der praktischen Anwendung erlernen.

Ziel des NLS-Kurses ist es, den Geburtshelfer in die Lage zu versetzen, eine Adaptationsstörung oder Asphyxie zu erkennen und Reanimationsmaßnahmen rechtzeitig und effektiv zu beginnen. Es soll das Hintergrundwissen und Fertigkeiten vermittelt werden, die ermöglichen, die ersten 10-20 Minuten nach einer Geburt zu überbrücken, gegebenenfalls bis ein neonatologisches Team angekommen ist (Baskett et al. 2005, S. 186). Der Teilnehmer sollte nach dem Kurs in der Lage sein, das Kind über Maskenbeatmung zu oxygenieren und einen intravenösen Zugang, z.B. über die Nabelvene, zu legen. Es wird der Umgang mit den wichtigsten Medikamenten vermittelt und auf Besonderheiten in der Physiologie und Anatomie, 
insbesondere auf ein mögliches Verletzungspotential z.B. durch hohe Beatmungsdrücke hingewiesen. Ständige Wiederholungen des Reanimationsschemas sollen den klaren Handlungsablauf vertiefen und einprägen.

Ein weiteres Ziel des Kurses ist es, die Partizipanten an das Thema „Reanimation“ heranzuführen und sie zu ermutigen, in einer offenen und freundlichen Atmosphäre Fragen zu stellen und Ängste abzubauen. Durch häufiges Feedback von den Instruktoren und häufige praktische Übungsmöglichkeiten soll das Zutrauen der Teilnehmer in die eigenen Fähigkeiten gestärkt werden und das Reanimationsschema verinnerlicht werden. Die Teilnehmer sollen lernen, Schwächen zu verbessern, vor allen Dingen aber ihre Stärken zu erkennen (Baskett et al. 2005, S. 182). Die Wichtigkeit der Kommunikation innerhalb der Gruppe soll betont werden, die Zusammenarbeit zwischen den Reanimierenden sollte gefördert werden und es sollte zu respektvoll-korrigierender Kommunikation auch entgegen der Hierarchiestufe angeregt werden (beispielsweise der Hinweis der Pflegekraft an den Arzt: „der Thorax hebt sich nicht, die Beatmung kommt nicht an").

In der gemischten Gruppe können Teilnehmer der verschiedenen Berufsgruppen voneinander lernen und andere Standpunkte kennen lernen. Letzten Endes sollen die Teilnehmer den Kurs mit einem gestärkten Selbstbewusstsein und Zutrauen in ihre Kenntnisse verlassen.

Um eine hohe Qualität der Neugeborenenreanimation auch in kleinen Krankenhäusern zu gewährleisten, wo das Personal eher selten einem Reanimationsereignis gegenübersteht (ÄKN 2007), ist es demnach notwendig, Reanimationskurse mit einem adäquaten Setting vorzuhalten, in denen sowohl die theoretischen und praktischen Kenntnisse der Teilnehmer aufgefrischt werden, als auch das Selbstbewusstsein der Partizipanten gestärkt wird. Diese Kurse sollten in einem adäquaten zeitlichen Abstand wiederholt werden, um den aufgebauten Wissensstand zu erhalten.

\subsubsection{Praktisches Vorgehen bei dieser Kursevaluation}

Wie bereits erörtert, kann eine Überprüfung, ob die theoretischen Kenntnisse im Rahmen der Schulung verbessert wurden und ob die Teilnehmer die Lerninhalte verstanden haben, anhand eines theoretischen Testes durchgeführt werden. In den Richtlinien zur Kursorganisation (Baskett et al. 2005, S. 186) ist eine Überprüfung der 
theoretischen Kenntnisse der Teilnehmer vor und nach dem Kurs anhand eines standardisierten Fragebogens vorgesehen. Aus diesem Fragebogen wurden für die vorliegende Arbeit diejenigen Fragen zur Auswertung ausgewählt, die für eine Reanimation relevantes Wissen und somit die wichtigsten Lerninhalte des NLSKurses abbilden. Diese Fragen sollten die Teilnehmer vor, direkt nach und sechs Monate nach der Schulung beantworten, um Wissensgewinn und Wissenserhalt nachvollziehen zu können.

Die Überprüfung der praktischen Fähigkeiten anhand einer Leitung und technisch korrekten Durchführung eines Reanimationsablaufes wird laut Richtlinien am Ende des Kurstages verlangt (Baskett et al. 2005, S. 186), um nicht nur das theoretische Wissen, sondern auch die praktische Umsetzung dieses Wissens überprüfen zu können. Alle 181 Personen, welche während der Dauer dieser Studie an den NLSKursen teilgenommen haben, bestanden im Rahmen des Reanimationskurses eine praktische Prüfung, in der sie eine Reanimationssequenz leiten mussten und dabei einen Helfer zur effektiven Reanimation auffordern und anleiten sollten.

Weitere praktische Prüfungen der Teilnehmer (beispielsweise vor dem Kurs) würden allerdings den zeitlichen Rahmen der Schulung überfordern. Sechs Monate nach dem Kurs ist eine solche praktische Prüfung logistisch unmöglich, da die Kursteilnehmer üblicherweise in verschiedenen Städten und Krankenhäusern arbeiten (siehe hierzu die vorstehenden Ausführungen zu der Unmöglichkeit einer Untersuchung zur Morbidität und Mortalität der Kinder vor und nach einem Reanimationstraining). Daher musste auf eine Bewertung der praktischen Fähigkeiten im Rahmen dieser Evaluation verzichtet werden. Aufgrund des Umstandes, dass alle Probanden die praktische Prüfung des Kurses bestanden haben, kann von der grundsätzlichen Fähigkeit, die Theorie auch in die Praxis umzusetzen, zumindest unmittelbar nach Ende des Kurses, ausgegangen werden. Somit führt die fehlende weitergehende praktische Überprüfung zwar zu einer Einschränkung in der Aussagekraft dieser Studie, jedoch können aufgrund der vorliegenden Ausgangslage trotzdem belastbare Ergebnisse gewonnen werden. Gleichwohl sollten praktische Überprüfungen vor und nach Teilnahme an Reanimationskursen in Folgeuntersuchungen dringend einbezogen werden.

$\mathrm{Ob}$ und inwieweit es gelungen ist, den Teilnehmern gegebenenfalls vorhandene Ängste vor Reanimationssituationen zu nehmen und das Zutrauen der Teilnehmer in 
die eigenen Fähigkeiten zu stärken, wird anhand eines anonymen Beurteilungsbogens untersucht. Neben einer reinen Punktevergabe bezüglich Kursaufbau und Organisation geben im Besonderen die freien Kommentare Zeugnis über das subjektive Empfinden der Teilnehmer. Hierbei ist es von besonderem Vorteil, dass die freien Äußerungen unabhängig von einer vorgegebenen Themenwahl aufgeschrieben werden. Somit wird den Partizipanten die Möglichkeit einer individuellen Schwerpunktsetzung gegeben.

Denjenigen, die den Fragebogen nach sechs Monaten zurücksandten, wurde ein schriftliches Feedback bezüglich der richtig und falsch beantworteten Fragen gegeben. Das jeweilige Feedback wurde von einem Teil der Teilnehmer spontan kommentiert. Da diese spontanen Äußerungen nur von einer kleinen Anzahl der Teilnehmer getroffen wurden, lassen sich diese Bemerkungen nicht ohne weiteres auf die Gesamtheit der Teilnehmer übertragen. Gleichwohl helfen sie bei der Beurteilung der Schwierigkeiten bei der Beantwortung einzelner Fragen. 


\subsection{Diskussion der Fragestellungen}

Es soll nun anhand der in der Studie gewonnenen Ergebnisse erörtert werden, ob ein Lernerfolg im Sinne eines (objektiven) Wissenszuwachses und eines gesteigerten Selbstbewusstseins durch die Schulung nachzuweisen ist, und wie lange dieser Effekt anhält. Es werden Ansatzpunkte aufgezeigt, wo Verbesserungspotential des Kursprogramms gesehen wird. Schließlich wird ein idealer Zeitraum für die Gültigkeit eines Reanimationszertifikates, somit also ein Zeitraum innerhalb dessen die Übung wiederholt werden sollte, bestimmt.

\subsection{1 Überprüfung des Lernerfolges}

Es sollte überprüft werden, ob ein (anhaltender) Lernerfolg nach der Kursteilnahme festzustellen ist und ob es Unterschiede zwischen den Teilnehmern verschiedener Berufsgruppen in Vorwissen und Wissensgewinn gibt.

Im Vergleich der Auswertung der Fragebögen vor und nach dem Reanimationskurs ist ein signifikanter Lernerfolg bezüglich des theoretischen Wissens feststellbar. Der Mittelwert der richtigen Antworten steigt von $81,4 \%$ auf 92,7\%, dabei sinkt die Standardabweichung von $11,3 \%$ auf $7,9 \%$, was auf eine Homogenisierung des Wissenstandes schließen lässt.

Auffallend nach Auswertung der ersten Fragebögen ist die hohe Zahl an richtigen Antworten bereits vor dem Kurs. Hebammen und Kinderkrankenschwestern geben bereits vor dem Kurs über $75 \%$ richtige Antworten. Betrachtet man die Teilnehmerstruktur der Kurse - zumeist erfahrene Geburtshelfer und Personen, die immer wieder in die Erstversorgung von Neugeborenen einbezogen werden - so könnte ein gutes Vorwissen ausschlaggebend für die hohe Zahl an richtigen Antworten sein.

Die Gruppe der Ärzte erzielt in den theoretischen Überprüfungen stetig bessere Werte, als Hebammen und Kinderkrankenschwestern. Bereits vor dem Kurs werden von innen mehr als $82 \%$ der Fragen richtig beantwortet. Das könnte an der unterschiedlichen Ausbildungsstruktur liegen: Ärzte erleben bereits im Studium einen hohen Anteil an theoretischer Ausbildung und eine diesen Kursfragen ähnlich gestaltete Prüfungsstruktur, und sind somit auf die Art der Fragestellung besser vorbereitet. 
Bereits nach sechs Monaten ist ein erheblicher Wissensverlust bei allen Teilnehmergruppen festzustellen. Mit 86,9\% an richtigen Antworten entspricht das Ergebnis sechs Monate nach der Schulung fast wieder der vor dem Kurs erreichten Punktzahl.

Im Vergleich der Berufsgruppen sieht man, dass bei Anästhesisten und Kinderkrankenschwestern der Wissensverlust am stärksten ist. Diese stehen in ihrem täglichen Arbeitsfeld bei der Neugeborenenversorgung nur in Sondersituationen in der Verantwortung, da für die Erstversorgung primär Hebammen, Gynäkologen und Pädiater verantwortlich sind. Fachintensiv-Kinderkrankenschwestern werden grundsätzlich ebenso in die Neugeborenenversorgung mit einbezogen. Die Pflegekräfte, die an diesen Kursen teilnehmen, arbeiten allerdings nur zu etwa 50\% im Intensivbereich, die anderen 50\% sind im Säuglingszimmer tätig und somit nur selten mit Reanimationssituationen konfrontiert. Dadurch wird die Statistik in diesem Punkt bezüglich der Pflegekräfte verwischt und in ihrer Aussagekraft gemindert. Unter den Pädiatern nehmen üblicherweise die in der Neonatologie arbeitenden oder im Kreißsaal verantwortlichen Kinderärzte an diesem Kurs teil, so dass der herausragend gute Wissenserhalt damit zu erklären ist, dass diese Berufsgruppe als erstes zu neonatologischen Notfällen gerufen wird. Folglich ist es für diese Gruppe besonders einfach, in der alltäglichen Praxis das Wissen aufrechtzuerhalten. Im mittleren Feld angesiedelt sind Gynäkologen und Hebammen. Diese werden zwar häufig mit Geburtssituationen, aber nur selten mit Notfällen konfrontiert. Gleichwohl kann dieser Personenkreis sein Wissen wohl aufgrund der häufigen Geburtssituationen gut erhalten (siehe Tabelle 17). Eine Korrelation zwischen Wissensverlust bei seltener Anwendung stellten bereits Bould et al. (2009) fest. Auch die Nutzung von Merkhilfen wie z. B. eines Flussbildes des Reanimationsablaufes konnte die Leistungen der in ihrer Studie getesteten Kandidaten nicht verbessern, weil die rasche Handlungsabfolge bei der Neugeborenenreanimation keine Zeit zur visuellen Überprüfung des Ablaufes lässt.

\begin{tabular}{|c|c|c|c|}
\hline Mittelwert (\%): & Vorher & Nachher & Nach 6 Monaten \\
\hline Gynäkologen & 82,2 & 94,1 & 88,2 \\
\hline Pädiater & 87,7 & 93,6 & 92,9 \\
\hline Anästhesisten & 82,4 & 96,4 & 85,3 \\
\hline Hebammen & 76,3 & 87,9 & 85,5 \\
\hline Kinderkrankenschwestern & 74,8 & 87,5 & 77,2 \\
\hline
\end{tabular}

Tabelle 17: Mittelwerte der Ergebnisse aufgeschlüsselt nach Berufsgruppen zu drei Zeitpunkten 
Die hier erzielten Ergebnisse decken sich mit Resultaten aus vorhergehenden Studien anhand des NRP-Kurses in Italien (Trevisanuto et al. 2005) und der Türkei (Duran et al. 2008b). Jene Reanimationskurse waren jeweils auf zwei bzw. drei Tage ausgelegt, der Unterricht wurde von ausgebildeten Instruktoren nach den Richtlinien der AHA/AAP gegeben. Beide Studienleiter kamen zu dem Ergebnis, dass trotz dieser intensiven Kurse der Wissensverlust nach sechs Monaten signifikant war und ein Auffrischungskurs nach weniger als 2 Jahren, am besten jährlich angeboten werden sollte.

Zusammenfassend ist festzuhalten, dass unabhängig von der Vorbildung ein deutlicher Wissenszuwachs durch die Teilnahme an einem NLS-Kurs erzielt wird. Dieses Wissen kann jedoch nicht über einen längeren Zeitraum ohne regelmäßige praktische Anwendung bzw. Auffrischung vorgehalten werden.

\subsubsection{Gibt es Themengebiete, die besondere Schwierigkeiten bereiten?}

Zur Evaluation des Wissenstandes wurden zu den wichtigsten Themenkreisen Fragen ausgewählt, die das Verständnis der Teilnehmer widerspiegeln sollten.

Im Bereich „Reanimationsablauf beim Neugeborenen“ wurden folgende Fragen gestellt:

1) „Der Heizstrahler ist wichtiger, als das Baby zu trocknen und einzuhüllen“,

2) „Herzmassage ist sinnvoll, bevor die Lunge sich aufgebläht hat",

3) „Die initiale Reanimation eines Frühgeborenen unterscheidet sich sehr von einer Reanimation bei einem Reifgeborenen".

Von diesen Fragen wurden die ersten beiden entsprechend dem Durchschnitt mehrheitlich richtig beantwortet. Die letzte Frage (Nummer 3) bereitete vermehrt Schwierigkeiten. Aus der Email-Korrespondenz anlässlich des persönlichen Abschneidens bei der Beantwortung des Fragebogens sechs Monate nach dem Kurs ergab sich, dass es hier zu einem Missverständnis gekommen war. Während sich die Frage auf den Ablauf des Reanimationsschemas bei einem gesunden Frühgeborenen bezieht, urteilten viele Teilnehmer nach dem subjektiven Empfinden. 
Diese Frage hätte mit dem Wort „Reanimationsschema“ statt „Reanimation“ klarer formuliert werden können.

Bezüglich des Themas „Herz/Kreislauf“ wurden folgende Fragen gestellt:

1) „Ein notfallmäßiger venöser Zugang ist am besten über die Nabelvene zu bekommen",

2) „Thoraxkompressionen sollten den Thorax 1/3 des Weges bis zum Rücken eindrücken",

3) „Der Haupteffekt von Adrenalin ist die Vasodilatation“.

Auch in diesem Themenkreis wurden die ersten beiden Fragen entsprechend dem allgemeinen Durchschnitt beantwortet. Die letzte Frage („Der Haupteffekt von Adrenalin ist die Vasodilatation") führte ebenfalls zu gehäuften Problemen bei den Teilnehmern. Sie wurde im Zeitverlauf zunehmend seltener richtig beantwortet (richtige Antworten vor dem Kurs: $86 \%$, direkt nach dem Kurs $81 \%$ und nach sechs Monaten 75\%). Die Rückmeldungen einiger Kursteilnehmer im Anschluss an die Übersendung der Auswertung des Fragebogens nach sechs Monaten lassen darauf schließen, dass von einigen Teilnehmern offensichtlich „Vasodilatation“ mit „Vasokonstriktion“ verwechselt wurde. Andere Teilnehmer verstanden die Bedeutung von „Vasodilatation“ nicht. Dieser Verlauf spricht dafür, dass die wenigen in diesem Kurs erwähnten Medikamente (Adrenalin, Glucose, Volumen und Sauerstoff) doch intensiver besprochen werden sollten. Andererseits sollten die in den Fragen verwendeten Fremdwörter übersetzt werden, um allen Teilnehmern gleich gute Bedingungen zur Beantwortung der Fragen zu bieten. 
Im Schwerpunkt „Atemwege des Neugeborenen“wurde eine hohe Anzahl an Fragen gestellt:

1) „Die Atmung stoppt üblicherweise, bevor der Kreislauf versagt",

2) „Ein Säugling mit Herzfrequenz unter 60/min muss sofort intubiert werden",

3) „Hypothermie kann Apnoen verursachen“,

4) „Maskenbeatmung ist viel weniger effektiv, als Beatmung nach Intubation",

5) „Es ist ratsam die Reanimation bei einem Frühgeborenen mit einem niedrigeren Beatmungsdruck zu starten, als bei einem reifen Neugeborenen“,

6) „Ein am Termin Geborenes kann mehr als $100 \mathrm{ml}$ Fruchtwasser ohne Hilfe beseitigen",

7) „Bronchiallavage verbessert immer die Prognose bei Mekoniumaspiration“,

8) „Systeme, die zur Beatmung von Neugeborenen benutzt werden, sollten ein Überdruckventil von $30-40 \mathrm{~cm}$ Wassersäule besitzen“,

9) „Schnappatmung ist immer flach“.

Die Fragen Nr. 1 bis 5 wurden entsprechend dem Durchschnitt häufig richtig beantwortet. Besonders häufig falsch beantwortet wurden die Fragen Nr. 6 bis 8 . Dabei wurde die Frage Nr. 8 zum Überdruckventil am Kursende zu 100\% richtig beantwortet. Das lässt darauf schließen, dass diese Frage didaktisch gut präsentiert wurde, jedoch die anderen beiden Themen im Kurs offensichtlich nicht gut vermittelt wurden. Die Frage Nr. 9 zur Schnappatmung wurde im Verlauf immer seltener richtig beantwortet (richtige Antworten vor dem Kurs: 81\%, direkt nach dem Kurs 77\% und nach sechs Monaten 71\%). Hier lag ein eindeutiges Verständnisproblem vor, das in den Vorträgen nicht geklärt werden konnte. 
Diese Ergebnisse sprechen dafür, dass es wichtig ist, die Fragen nach dem Kurs zu besprechen, um so zu Diskussionen anzuregen und Verständnisprobleme vor Ende des Kurstages klären zu können.

Die Fragen zum Bereich der „Post-Reanimationsphase“

1) „Alle Kinder, die bei Geburt intubiert werden müssen auf eine Neugeborenenintensivstation übernommen werden",

2) „Nach einer Reanimation ist wichtig: Abnahme von Nabelschnurblut und venösem Blut zur Messung von $\mathrm{pH}$ und $B E^{*}$,

wurden alle entsprechend dem Ergebnisdurchschnitt beantwortet, was darauf schließen lässt, dass dieser Bereich gut verstanden wurde.

Zumal eine Vielzahl von NLS-Kursen mit jeweils wechselnden Referenten evaluiert wurde, lassen sich die Ergebnisse nicht zwingend mit einer schlechten Vermittlung des Lernstoffes erklären. Bei künftigen Kursen wird jedoch verstärkt darauf zu achten sein, dass die Begrifflichkeiten eindeutiger erklärt bzw. die Einordnung bestimmter Vorgänge in den entsprechenden Kontext vorgenommen werden. Insbesondere die Fragen zum Themenbereich der medikamentösen Therapie wie die Funktionsweise von Adrenalin oder die Bedeutung von Bronchiallavage wurde von Hebammen und Pflegepersonal auffällig häufig falsch beantwortet. Aufgrund der jeweils kleinen Fallzahl an Antworten lässt sich hier keine belastbare Statistik berechnen. Ein entsprechender Trend wird aber auch im folgenden Abschnitt 5.3.4 deutlich, in dem Hebammen und Pflegepersonal den Kurs als sehr kompakt beschreiben und sich einen zweitägigen Kurs wünschen.

Um das Verständnis zu verbessern und wichtige Informationen besser einzuprägen, könnte beispielsweise schriftliches Material insbesondere zu den angewandten Medikamenten (Adrenalin, Glucose, Volumen und Sauerstoff) sowie der zu beachtenden Grenzwerte (z. B. Überdruckventil am Beatmungsbeutel, Beatmungsdrücke bei Früh- und Neugeborenen) ausgegeben werden, damit diese Informationen nach den Kurs nachgelesen werden können.

Eine anschließende Besprechung der Fragebögen nach dem Kurs könnte strittige Fragen klären und zu Diskussionen hinsichtlich der noch nicht vollständig 
verstandenen Themen führen. So würden Verständnisprobleme noch innerhalb des Kurses aufgedeckt und geklärt.

Der Fragebogen zum Kurs ist standardisiert und soll daher nicht von einzelnen Ausbildern verändert werden. Veränderungen im Fragebogen müssen daher verbindlich für alle NLS-Kurse vom ERC ausgegeben werden. Da die Fragen aber offensichtlich immer wieder zu Missverständnissen führen oder einzelne Worte (Fachworte wie „Vasodilatation“) nicht verstanden werden, sollte der Fragebogen dringend überarbeitet werden.

\subsubsection{Konnte der Kurs das Selbstbewusstsein der Teilnehmer stärken und das Vertrauen in die persönlichen Fähigkeiten erhöhen?}

Entsprechend den Ergebnissen der Studie von Verplancke et al. (2008) sollte ein Reanimationskurs darauf hin zielen, das Selbstbewusstsein der Teilnehmer zu stärken. Es konnte vielfach nachgewiesen werden, dass praxisnahe Simulationsübungen zu einem erhöhten Vertrauen in die eigenen Fähigkeiten beitragen (Russo et al. 2007, Cooke et al. 2008, Sørensen et al. 2009).

Der hohe Anteil an praktischen Übungen, insbesondere das Simulationstraining mit realistischen Situationen wurde von den Teilnehmern der hier untersuchten Kurse als positiv empfunden. In der Gesamtbeurteilung wurde die Schulung von nahezu allen Teilnehmern mit "sehr gut" benotet. In den spontanen Kommentaren wird die Atmosphäre im Kurs als offen und locker beschrieben. Die Dozenten seien engagiert und freundlich, es wird immer wieder darauf hingewiesen, dass die Teilnehmer ein konstruktives Feedback erhalten. Mehrere Teilnehmer fühlen sich nach dem Kurs in ihrem Selbstbewusstsein gestärkt, hatten mehr Zutrauen in Ihr eigenes Können und gaben das Gefühl an, „ein Schema für den Notfall im Kopf“ zu haben. Die Zusammenarbeit mit den Kollegen würde in diesem Kurs gefördert.

Folglich wurden durch den Kurs die Grundlagen geschaffen, dass sich die Teilnehmer selbstbewusst einer Reanimationssituation nähern und effektiv mit ihren Kollegen kommunizieren. 


\subsubsection{Gibt es wiederkehrende Kritikpunkte in der subjektiven Bewertung der Kurse?}

Hinsichtlich der Fragebögen kam wiederholt die Kritik auf, dass die Fragen missverständlich seien (insgesamt 18 Teilnehmer äußerten diese Kritik, das entspricht $1 \%$ der befragten Teilnehmer), etwa ebenso viele Teilnehmer baten um Lösungsblätter zu den Fragen. Lösungsblätter wurden im Kurs bewusst nicht ausgegeben, um das Ergebnis der Auswertung nach sechs Monaten nicht zu verfälschen. Wie aber bereits in Abschnitt 5.3.2 besprochen scheint es dringend notwendig, die Fragebögen mit den Teilnehmern am Ende des Kurstages durchzusprechen und eine Überarbeitung der Fragebögen anzustreben.

Ein weiterer wiederkehrender Kritikpunkt betraf die Kursdauer, wobei die Meinungen hinsichtlich der optimalen Kursdauer unter den Teilnehmern weit auseinander gehen. Um eine anhaltend gute Konzentrationsfähigkeit der Partizipanten aufrecht zu halten, sollte ein Reanimationskurs nicht zu kurz und komprimiert, aber auch nicht zu lang und ausschweifend sein.

Hebammen und Kinderkrankenschwestern empfanden den Kurs zumeist als kurz und komprimiert. Zudem wurde von dieser Gruppe auch der Wunsch nach mehr Theorie geäußert (siehe auch Abschnitt 5.3.2) und wiederholt vorgeschlagen, die Unterrichtszeit von acht Stunden auf zwei Tage auszudehnen.

Hinsichtlich der Ausdehnung der Kursdauer auf zwei Tage muss jedoch beachtet werden, dass mit der Besetzung von Räumen, Bezahlung der Dozenten und auch der Anreise und dem Aufenthalt der Teilnehmer von mehr als 10 Stunden zusätzliche Kosten anfallen. Zudem ist es für die meisten Teilnehmer und Dozenten aufgrund ihrer Tätigkeit im Schichtdienst oftmals nicht einfach, für eine zweitägige Fortbildung freigestellt zu werden.

Anästhesisten, Gynäkologen und Pädiater empfanden die Schulung demgegenüber eher als zu lang, aber bezüglich des eigenen Fachbereiches zu unspezifisch. Mehrfach wurde der Wunsch nach einer intensiveren Behandlung der Themen „medikamentöse Therapie“, „weiteres Vorgehen nach einer Reanimation“ sowie nach mehr praktischen Übungen zur Intubation geäußert. 
Hauptziel des Kurses ist es, den Kursteilnehmern ein klares, knappes Reanimationsschema mitzugeben, das für diese auch in Stresssituationen noch abrufbar ist. Daher werden beispielsweise Themenbereiche wie "Medikamente" entsprechend den "Richtlinien für Neugeborenenreanimation“ auf Adrenalin, Volumen, Glucose und Sauerstoff beschränkt (AHA 2005, Baskett et al. 2005 s.119122). Auf weiterführende Vorträge wird bewusst verzichtet, da diese das Ziel der Fortbildung überschreiten und zudem nicht zur Festigung der Reanimationsprinzipien beitragen.

Im Rahmen des „Airway-Management“ wird während der Übungseinheiten ein deutlicher Schwerpunkt auf Maskenbeatmung unter Vernachlässigung der Intubation gelegt, weil das Risiko einer Atemwegsobstruktion nach mehrfachen Intubationen durch einen ungeübten Ersthelfer deutlich zunimmt. Es ist zweifelhaft, ob ein Geburtshelfer, der nur sehr selten in die Situation kommt, ein Neugeborenes zu reanimieren und $z u$ intubieren, bereits nach wenigen Übungen an einer Puppe ausreichend Intubationskenntnisse erwerben kann, um sicher ein Neugeborenes zu behandeln. In den USA an Puppen trainierte Rettungsassistenten zeigten eine Rate an erfolgreichen Intubationen am lebenden Menschen von 53\% (Sayre et al. 1998). Daher kann nicht davon ausgegangen werden, dass eine Intubation in einem eintägigen Kurs erlernbar wäre. Eine effektive Maskenbeatmung ist jedoch bei fast allen Neugeborenen möglich (Palme-Kilander 1992).

Die teilweise weit auseinander reichenden Wünsche der unterschiedlichen Berufsgruppen lassen die Frage aufkommen, ob es nicht vorteilhafter wäre Hebammen, Pfleger und Ärzte (ggf. weiter unterteilt nach Fachrichtungen) getrennt zu unterrichten.

Für diesen Vorschlag spricht, dass es in berufsgetrennten Kursen einfacher ist, auf berufsspezifische Wünsche einzugehen und entsprechende Schwerpunkte zu setzen. Zudem besteht in Deutschland eine klare Hierarchie zwischen Vorgesetzten und nachgeordnetem Personal, was eine gleichberechtigte Zusammenarbeit sowohl im Kurs, als auch im Alltag erschwert. In einer Studie von Walker and Balvert (1994) bezüglich Schulungen für Neugeborenenreanimation gab das Pflegepersonal an, die Übungssituationen gemeinsam mit den Ärzten (des eigenen Krankenhauses) als stressbelasteter zu empfinden, als ohne Ärzte. In den NLS-Kursen selbst wird versucht, diese hierarchische Situation zu entschärfen, indem die Kleingruppen 
gemischt aus Teilnehmern der unterschiedlichen Berufsgruppen aus verschiedenen Krankenhäusern zusammengestellt werden. Auf diese Weise wird die Konfrontation mit der/dem eigenen Vorgesetzten während der praktischen Übungen vermieden.

Gegen eine Trennung der Berufsgruppen kann eingewandt werden, dass im Alltag eine klare und selbstbewusste Kommunikation zwischen den Geburtshelfern der unterschiedlichen Hierarchiestufen zwingend vonnöten ist (siehe auch Abschnitt 5.2.1), und diese bereits in den gemischten Gruppen ohne hierarchischen Druck während der praktischen Lerneinheiten im Kurs trainiert werden kann.

Weiterhin besteht in einem gemischten Team die Möglichkeit des „voneinander Lernens". Durch die jahrelange und vielschichtige Berufserfahrung der Teilnehmer unterschiedlicher Berufsgruppen, werden während eines jeden Kurses vielfältige Aspekte diskutiert und aus diversen Standpunkten dargestellt, so dass diese Diskussionen eine Bereicherung für alle Kursteilnehmer darstellen.

Trotz der weit auseinander reichenden Vorstellungen der verschiedenen Berufsgruppen wurden die Referenten des Kurses von allen Teilnehmern als sehr kompetent eingeschätzt und die Präsentation der Informationen positiv bewertet. Pädiater und Anästhesisten zogen einen niedrigeren subjektiven Gewinn aus den Vorträgen. Diese Gruppe zeigte aber auch in der Auswertung der Berufsgruppen die höchsten theoretischen Vorkenntnisse und konnte so aus den Vorträgen sicher weniger neue Erkenntnisse ziehen.

Der subjektiv empfundene persönliche Nutzen korreliert positiv mit der Häufigkeit an Notfällen im beruflichen Alltag. So zogen Hebammen, die eher gesunden Neugeborene auf die Welt helfen (siehe auch Abschnitt 1.1), einen eher geringen subjektiven Nutzen aus dem Kurs, Pädiater dagegen empfanden den Kurs als sehr hilfreich. Die Rate an reanimationsbedürftigen Neugeborenen schwankt aufgrund der Vorselektion je nach Größe der geburtshilflichen Einrichtung zwischen 1,7\% und 10\% (ÄKN 2007, SLÄK 2008). Dies erklärt, warum das subjektive Empfinden über die Häufigkeit von Reanimationen enorm variiert und entsprechend mehr oder minder Profit aus einer solchen Fortbildung gezogen werden kann.

Trotzdem fühlen sich fast alle Teilnehmer angeregt, Aspekte ihrer medizinischen Tätigkeit zu ändern oder zu überdenken. Diese Antwort ist umso positiver zu 
bewerten, als die Partizipanten meist langjährig in ihrem Beruf tätig sind und dort bereits viele Situationen meistern mussten, und auch Berufsanfänger in die Arbeit eingewiesen haben. Gerade bei diesen oftmals sehr erfahrenen Geburtshelfern ist diese selbstkritische Haltung als ein guter Erfolg des Kurses zu werten.

\subsubsection{Wie kann man die Kursqualität verbessern?}

Der Kursaufbau des NLS-Kurses folgt den Richtlinien des ERC (Baskett et al. 2005 S. 181-189), die einen hohen Anteil an praktischer Übung in Kleingruppen (4-8 Personen) mit einem Verhältnis von Instruktoren zu Teilnehmern von 1:3 (im Rahmen des NLS-Kurses) vorsehen. Für die Kursdauer sind acht Stunden vorgesehen; die Schulung ist für jegliches medizinisches Fachpersonal, das bei einer Geburt involviert werden könnte, ausgerichtet.

Die Instruktoren werden vom ERC nicht nur inhaltich, sondern insbesondere didaktisch mit dem Ziel ausgebildet, eine offene Lernatmosphäre zu schaffen um Hemmungen und Ängste bei den Lernenden zu minimieren.

Von den Teilnehmern wird erwartet, dass sie sich vor dem Kurs Wissen anhand eines Buches oder einer CD aneignen. Bisher haben die Teilnehmer vor dem Kurs keine Materialien erhalten. Dies liegt zum einen daran, dass derzeit noch keine offizielle Übersetzung des Handbuches, welches die angesprochenen Themen umfangreich behandelt (Richmond 2008), aus der englischen in die deutsche Sprache vorliegt, zum anderen daran, dass die Kosten des Kurses mit Aushändigung dieses Buches um $16 \%$ steigen würden, diese Kostensteigerung jedoch für viele Teilnehmer nicht akzeptabel wäre. Trotzdem sollte die vorhandene Ausgabe des Handbuches in Englisch (Richmond 2008) aktiv angeboten werden, z.B. als zusätzliche Bestellmöglichkeit im Rahmen der Anmeldung. Das baldige Angebot einer CD als Vorbereitungsmaterial für den Kurs sollte angestrebt werden, um den Teilnehmern eine Vorbereitung auf den Kurs zu ermöglichen.

Es ist sinnvoll die Fragen der Testate am Ende des Kurstages zu besprechen, um Missverständnisse aus dem Weg zu räumen. Letztlich sollten die Teilnehmer während des Kurses neben dem Reanimationsschema ein Thesenpapier mit wichtigen Informationen zu Medikamenten und Beatmungsdrücken ausgeteilt bekommen, um innen wenigstens die wichtigsten Informationen schriftlich mitzugeben. 
Im Rahmen der geplanten Überarbeitung des Kurses 2010 entsprechend den dann bestehenden neuen Richtlinien des ERC sollten die Fragebögen, wie bereits in Abschnitt 5.3.2 angesprochen, überarbeitet werden.

Trotz der in den letzten Abschnitten dargestellten Kritik an Kursdauer und Kursinhalt ist eine Änderung in diesen Bereichen genauer abzuwägen. Zum einen gehen die Meinungen der Teilnehmer bezüglich dieser Themen weit auseinander, so dass bei der gemischten Teilnehmergruppe eine Änderung in jedwede Richtung keine Verbesserung für die Gesamtgruppe darstellt. Zum anderen wurden die Kursstandards ausgearbeitet, um bei gleich bleibender Kursqualität den Reanimationsablauf beim Neugeborenen klar und einprägsam zu vermitteln. Weitergehende Kursinhalte würden das Ziel überschreiten, ohne zur Festigung des Flussschemas der Reanimation beizutragen. Vielmehr würde eine Vielzahl an Informationen und Handlungsmöglichkeiten in einer solchen Stresssituation eher zur Verwirrung beitragen und damit die Qualität des Reanimationsablaufes mindern.

Eine Heterogenität der Teilnehmer sollte beibehalten werden, da die verschiedenen Berufsgruppen im Alltag konstruktiv miteinander arbeiten müssen und die Auseinandersetzung mit anderen Kollegen aus anderen Berufsfeldern oder Hierarchieebenen einen Teil der Lernziele darstellt.

Um diese Zusammenarbeit weiter zu fördern, könnte ein stärkerer Schwerpunkt auf die Förderung einer respektvollen, klaren und selbstbewussten Kommunikation zwischen den Reanimierenden gelegt werden. Durch gute Kommunikation, Besprechungen nach einer Reanimation und ein anonymes Fehlermeldesystem können viele medizinische Fehlhandlungen erkannt und vermieden werden (Finer and Rich 2002).

\subsubsection{In welchem Zeitraum ist eine Wiederholung des Kurses empfehlenswert?}

Die Teilnehmer des NLS-Kurses erhalten nach bestandener theoretischer und praktischer Prüfung am Ende des Kurstages ein Zertifikat mit einer Gültigkeit von fünf Jahren.

Die in dieser Untersuchung gewonnenen Ergebnisse betätigen die Ergebnisse der vorangegangenen Untersuchungen über den NRP-Kurs (Dunn et al. 1992, 
Trevisanuto et al. 2005, Duran et al. 2008b), in denen ebenfalls ein Wissensverlust bereits nach sechs Monaten nachgewiesen werden konnte.

In anderen Studien wurde neben dem vorstehend beschriebenen Wissensverlust auch eine Minderung der praktischen Fähigkeiten, bzw. die unvollständige Durchführung des Reanimationsablaufes sechs bis zwölf Monate nach dem NRPKurs nachgewiesen (Duran et al. 2008b, Verplancke et al.2008, Bould et al.2009). Demzufolge kann davon ausgegangen werden, dass auch bei den Teilnehmern des NLS-Kurses die praktischen Fähigkeiten nachgelassen haben. Auch wenn der Arbeitsalltag einiger Teilnehmer den Wissensverlust verlangsamt (in unserer Studie deutlich zu sehen in der berufsgruppenabhängigen Auswertung der Testbögen bei der Gruppe der Pädiater), so ist dieses jedoch kein Ersatz für die regelmäßige Auffrischung der Kenntnisse.

Letztlich ist zu beachten, dass sich auch die Reanimationsempfehlungen in stetem Wandel befinden. Dies spiegelt sich auch in der Aktualisierung der Reanimationsrichtlinien alle fünf Jahre wider (Chamberlain et al. 2005, Kattwinkel 2008).

Unter Berücksichtigung der oben genannten Ergebnisse sollte ein Reanimationskurs bei geburtshilflich involviertem Personal im Idealfall halbjährlich, möglichst aber jährlich wiederholt werden. Dies dient dann nicht nur der Aufrechterhaltung der Fähigkeiten, sondern auch der Aktualisierung der Kenntnisse.

Dafür wäre es sinnvoll, einen speziellen, ebenfalls standardisierten Auffrischungskurs von entsprechend kürzerer Dauer für Teilnehmer mit Vorkenntnissen zu entwickeln, der dann jährlich wiederholt werden könnte. Ein derartig angepasster Auffrischungskurs könnte durch einen geringeren Kosten- und Zeitaufwand zu einer höheren Akzeptanz bei den Kliniken führen und so die Durchsetzung der jährlichen Wiederholung des Reanimationskurses erleichtern. 


\section{Zusammenfassung}

In der vorliegenden Arbeit wurde ein in Deutschland nach den Leitlinien des ERC angebotener Neugeborenreanimationskurs für Geburtshelfer (Gynäkologen Hebammen, Pflegepersonal, Anästhesisten und Pädiater) hinsichtlich seiner Effektivität für eine spätere Reanimationstätigkeit untersucht. Es sollte im Anschluss eine Aussage darüber getroffen werden, welche Verbesserungsmöglichkeiten bestehen und zu welchem Zeitpunkt eine Auffrischung des Kurses zu empfehlen sei.

Als zu überprüfende Ziele des Reanimationskurses wurden Wissensgewinn, Wissenserhalt, sowie Gewinn an Selbstvertrauen definiert.

Die praktische Prüfung der Fertigkeiten direkt nach dem Kurs im Rahmen des Abschlusstestes wurde von allen Teilnehmern bestanden. Eine Evaluation der praktischen Fähigkeiten vor und Monate nach dem Kurs konnte aus logistischen Gründen nicht durchgeführt werden. Dies stellt eine gewisse Einschränkung in der Aussagekraft der Studie dar und sollte in einer späteren Studie evaluiert werden.

Es konnte ein signifikanter Wissensgewinn bei Teilnehmern aller Berufsgruppen nach dem Kurs nachgewiesen werden. Bereits nach sechs Monaten war ein signifikanter Wissensverlust fast auf Ausgangsniveau nachweisbar. Dieser Wissensverlust korreliert negativ mit der täglichen Anwendungsmöglichkeit der Kenntnisse. In kleinen Krankenhäusern ohne pädiatrische Abteilung (und dadurch wenigen Notfällen) ist daher ein größerer Wissensverlust zu erwarten, als in einem Perinatalzentrum mit einem höheren Prozentsatz an Notfällen.

Die meisten Teilnehmer empfanden den Kurs als hilfreich, fühlten sich nach dem Kurs in ihrem Selbstbewusstsein gestärkt und gaben an, einige Vorgehensweisen in ihrem Berufsalltag ändern zu wollen.

Alle behandelten Themengebiete wurden von den Teilnehmern inhaltlich gut verstanden, es kam jedoch wiederholt zu Missverständnissen in der Beantwortung der Fragen des Fragebogens. Fachausdrücke wie "Vasodilatation" wurden nicht von allen Teilnehmern verstanden. Daher ist eine Nachbesprechung der Fragebögen am Ende des Kurstages sinnvoll. Zudem können im Rahmen einer solchen Nachbesprechung eine erneute Diskussion angeregt und letzte Fragen geklärt 
werden. Die standardisierten Fragebögen sollten nochmals überarbeitet werden. Dabei sollten missverständliche Fragestellungen verbessert und Fachausdrücke übersetzt werden.

Da das Handbuch zum Kurs (Richmond 2008) bisher aus dem Englischen nicht ins Deutsche übersetzt wurde und nicht im Kurspreis enthalten ist, sollte eine Alternative gefunden werden, um den Teilnehmern eine Möglichkeit der selbstständigen Vorund Nachbereitung zu geben. Zur Vorbereitung könnte den Teilnehmern Material in Form einer $C D$ zur Verfügung gestellt werden. Zusätzlich zum bereits im Kurs ausgeteilten Handzettel mit dem Flussschema des Reanimationsablaufes, sollten schriftliche Informationen über wichtige Grenzwerte und Medikamentendosierungen ausgegeben werden.

Kritik am Kurs wurde häufig bezüglich der Kursdauer geäußert, dabei war es berufsabhängig, ob der Kurs als sehr lang und unspezifisch oder zu kurz und zu komprimiert empfunden wurde. Eine Beibehaltung der Kursorganisation ist sinnvoll, da die zu behandelnden Themen nicht in kürzerer Zeit zu erfassen sind, dagegen aber eine Verlängerung der Kursdauer nicht von der Mehrheit der Teilnehmer aufgrund höherer Kosten und höheren Zeitaufwands getragen werden kann bzw. wird.

Pädiater und Anästhesisten baten um eine Vertiefung der Kursinhalte hinsichtlich der Themen Intubation und Medikamente. Eine Beibehaltung der Kursinhalte in ihrer Allgemeinheit ist dagegen sinnvoll. Aufgrund der häufigen Wiederholungen des Reanimationsschemas werden die vermittelten Inhalte verinnerlicht. Dies ist notwendig, da in einer Reanimationssituation keine Zeit bleibt, den Handlungsablauf nachzulesen. Hingegen ginge eine Vertiefung dieser beiden Themen über das Kursziel hinaus und würde die Übungsmöglichkeiten der anderen Teilnehmer einschränken.

Eine Trennung der Teilnehmer entsprechend ihrer Berufsgruppe und das Angebot eines berufsspezifischen Kurses ist nicht sinnvoll. In gemischten Gruppen sollen die Teilnehmer voneinander lernen, die Kommunikation untereinander üben und letztlich als Team miteinander agieren. 
Mit Blick auf den rapiden Wissensverlust bei unregelmäßiger Anwendung der Kenntnisse sollte in jeder geburtshilflichen Abteilung zumindest einmal jährlich ein Neugeborenen-Reanimationskurs angeboten werden. Dazu sollte ein Auffrischungskurs mit entsprechend verkürzter Stundenzahl entwickelt werden.

In Folgestudien bleibt zu evaluieren, ob mit dem theoretischen Wissensverlust auch eine Verminderung der praktischen Fertigkeiten einhergeht.

Sobald wie möglich sollte zudem eine Studie betreffend die Mortalität und Morbidität vor und nach Einführung eines Reanimationskurses durchgeführt werden, da nur so die tatsächliche Effektivität dieser Fortbildung gemessen werden kann.

\section{Zusammenfassend lassen sich folgende Kernthesen festhalten:}

Die Teilnahme an einem NLS-Kurs nach den Richtlinien des ERC führt bei den Partizipanten zu einem Wissenszuwachs, welcher zusammen mit einer Erhöhung des Selbstbewusstseins der Kursteilnehmer einen qualitativ guten Reanimationsablauf und damit ein gutes Outcome der zu reanimierenden Neugeborenen erwarten lässt.

1. Eine Verbesserung der Effektivität des bisherigen Kurskonzeptes kann insbesondere durch eine Verstärkung der aktiven und passiven Wissensvermittlung anhand von Vorbereitungsmaterial und einer Diskussion der Fragen am Ende es Kurses erreicht werden. Der Fragebogen sollte zur besseren Verständlichkeit überarbeitet werden. Zusätzlich sollte die Bedeutung der Kommunikation stärker betont werden und mehr Wert auf das Einüben von Kommunikationsabläufen gelegt werden.

2. Da bereits nach 6 Monaten ein deutlicher Wissensverlust feststellbar ist, sollte zur Aufrechterhaltung der theoretischen und praktischen Fähigkeiten eine erneute Kursteilnahme alle sechs Monate, mindestens aber jährlich erfolgen.

3. Dafür sollte ein standardisierter Auffrischungskurs mit entsprechend verkürzter Kursdauer entwickelt werden. 


\title{
7. Anhang
}

\section{Anhang 7.1 Frageboten vor dem Kurs 2008}

\author{
Deutscher Rat für Wiederbelebung im Kindesalter e.V. \\ German Resuszitation Concil e.V. \\ www.dr-wiki.de
}

\section{Newborn Life Support Kurs \\ Prätest}

Bitte mit $R=$ richtig oder $F=$ falsch markieren .

1 Der Heizstrahler ist wichtiger, als das Baby zu trocknen und einzuhüllen.

2 Ein notfallmäßiger venöser Zugang ist am besten über die Nabelvene zu bekommen.

3 Der Haupteffekt von Adrenalin ist die Vasodilatation.

4 Bronchial Lavage verbessert immer die Prognose bei Mekoniumaspiration.

5 Herzmassage ist sinnvoll, bevor die Lunge sich aufgebläht hat.

6 Die Atmung stoppt üblicherweise bevor der Kreislauf versagt.

7 Ein am Termin Geborenes kann mehr als $100 \mathrm{ml}$ Fruchtwasser ohne Hilfe beseitigen.

8 Ein Säugling mit einer Herzfrequenz unter 60/min muss sofort intubiert werden.

9 Thorax Kompressionen sollten den Thorax 1/3 des Weges bis zum Rücken eindrücken.

10 Systeme, die für eine Beatmung von Neugeborenen genutzt werden, sollten ein Überdruckventil von 30-40 cm Wassersäule besitzen.

11 Alle Kinder, die bei Geburt intubiert werden, müssen auf eine Neugeborenenintensivstation übernommen werden.

12 Hypothermie kann Apnoen verursachen.

13 Es ist ratsam die Reanimation eines Frühgeborenen mit einem niedrigerem Beatmungsdruck zu starten als bei einem reifen Neugeborenen.

14 Maskenbeatmung ist viel weniger effektiv als Beatmung nach Intubation.

15 Die initiale Reanimation eines Frühgeborenen unterscheidet sich sehr von einer Reanimation bei einem Reifgeborenen.

16 Schnappatmung ist immer flach.

17 Nach einer Reanimation ist wichtig: Abnahme von Nabelschnurblut und venösem Blut für $\mathrm{pH}$ und Base Excess Messungen. 


\section{Anhang 7.2 Frageboten vor dem Kurs 2009}

\section{Deutscher Rat für Wiederbelebung im Kindesalter e.V. \\ German Resuszitation Concil e.V. \\ www.dr-wiki.de}

\section{Newborn Life Support Kurs \\ Prätest}

Bitte mit $\mathrm{R}=$ richtig oder $\mathrm{F}=$ falsch markieren .

1 Atmung stoppt nach 2-3 Minuten sobald das Gehirn keinen Sauerstoff mehr bekommt = primäre Apnoe.

2 Wenn Adrenalin gebraucht wird, ist die Situation oft schlecht*

3 Der Apgar Score hat keinen direkten Wert für den Ablauf einer Neugeborenen Reanimation*

4 Der Heizstrahler ist wichtiger, als das Baby zu trocknen und einzuhüllen

5 Ein notfallmäßiger venöser Zugang ist am besten über die Nabelvene zu bekommen

6 Der Haupteffekt von Adrenalin ist die Vasodilatation

7 Bronchial Lavage verbessert immer die Prognose bei Mekoniumaspiration

8 Herzmassage ist sinnvoll, bevor die Lunge sich aufgebläht hat

9 Die Atmung stoppt üblicherweise bevor der Kreislauf versagt

10 Ein am Termin Geborenes kann mehr als $100 \mathrm{ml}$ Fruchtwasser ohne Hilfe beseitigen

11 Ein Säugling mit einer Herzfrequenz unter 60/min muss sofort intubiert werden

12 Thorax Kompressionen sollten den Thorax 1/3 des Weges bis zum Rücken eindrücken

13 Systeme, die für eine Beatmung von Neugeborenen genutzt werden, sollten ein Überdruckventil von 30-40 cm Wassersäule besitzen

14 Alle Kinder, die bei Geburt intubiert werden, müssen auf eine Neugeborenenintensivstation übernommen werden

15 Hypothermie kann Apnoen verursachen

Es ist ratsam die Reanimation eines Frühgeborenen mit einem niedrigerem Beatmungsdruck zu starten als bei einem reifen Neugeborenen

16 Maskenbeatmung ist viel weniger effektiv als Beatmung nach Intubation

17 Die initiale Reanimation eines Frühgeborenen unterscheidet sich sehr von einer Reanimation bei einem Reifgeborenen

18 Schnappatmung ist immer flach

19 Nach einer Reanimation ist wichtig: Abnahme von Nabelschnurblut und venösem Blut für $\mathrm{pH}$ und Base Excess Messungen 


\title{
Anhang 7.3 Fragebogen nach dem Kurs 2008-2009
}

\author{
Deutscher Rat für Wiederbelebung im Kindesalter e.V. \\ German Paediatric Resuscitation Council \\ www.dr-wiki.de
}

\section{Ausbildungszentrum für Pädiatrische Notfallmedizin Rheinland Kinderkrankenhaus St. Nikolaus Viersen \\ www.kinder-notfallkurse.de}

\section{Newborn Life Support Kurs \\ Abschlusstest}

Bitte im Antwortbogen ankreuzen unter $R=$ richtig oder $F=$ falsch

1 "Fetaler Distress" und "Geburtsasphyxie" sind nützliche Begriffe zur Dokumentation in den Akten.

2 Schnappatmung beginnt unmittelbar nachdem die normale Atmung stoppt.

3 Eine primäre und terminale Apnoe kann durch Anwendung des Apgar-Scores aufgedeckt werden.

4 Gesunde Neugeborene dürfen bis zu drei Minuten brauchen um mit der Atmung zu beginnen.

5 Absaugen des Rachens ist eine gute Methode die Atmung zu stimulieren.

6 Laktat-Anreicherung verursacht Störungen in der Herzfunktion.

7 Atemstimulantien wie Theophyllin sind hilfreich und sicher in der Neugeborenenreanimation.

8 Guedel Tuben werden in der Neugeborenenreanimation grundsätzlich nicht genutzt.

9 Frühgeborene unter 25 Wochen sollten nicht wiederbelebt werden.

10 Initiale Beatmung sollte eine Rate von 30-40 pro Minute betragen.

11 Bei terminaler Apnoe ist eine steigende Herzfrequenz ein Zeichen des Reanimationserfolges. 
12 Maskenbeatmung verursacht üblicherweise einen Pneumothorax.

13 Ca. $5 \%$ der Neugeborenen brauchen eine Intubation bei Geburt.

14 Ein apnoeisches Neugeborenes soll sofort Naloxon (Narcanti) erhalten.

15 Intubation ist essentiell für eine effektive Reanimation.

16 Bicarbonat ist ein Hauptbestandteil bei der NG-Reanimation und darf über den Tubus gegeben werden.

17 Calcium ist ein hilfreiches Medikament bei der neonatalen Reanimation.

18 Neugeborene ohne Auswurfleistung des Herzens werden azidotisch.

19 Erfassen der Zeit ist eine essentielle Maßnahme bei jeder Erstversorgung eines Neugeborenen.

20 Ein guter Ausgang kann erwartet werden, wenn normales Atmen nach Ablauf von 30 Minuten auftritt.

21 Blutzuckerbestimmung ist wichtig nach einer lang dauernden Reanimation.

22 Pneumothorax ist ein häufiger Grund für Probleme bei Geburt.

Nach einer Reanimation ist wichtig:

23 Genaue Dokumentation.

24 Information an die Eltern über den Hergang der Reanimation.

25 Sofortige Information an einweisenden Arzt.

26 Diskussion der Situation mit dem Team.

27 Die postnatale kardio-vaskuläre Adaptation hängt von der Aufblähung der Lungen bei Geburt ab.

28 Alle Sekretionen zu entfernen ist sehr wichtig einen freien Atemweg sicher zu stellen.

29 Eine Mekoniumaspiration verursacht häufig Probleme.

30 Luft ist weniger effektiv als Sauerstoff für eine Reanimation bei Geburt.

31 Atmung stoppt nach 2-3 Minuten sobald das Gehirn keinen Sauerstoff mehr bekommt = primäre Apnoe.

32 Wenn Adrenalin gebraucht wird, ist die Situation oft schlecht.

Deutsche Rat für Wiederbelebung im Kindesalter e.V. - Pädiatrische Arbeitsgruppe des GRC NLS Prätest Fragen D 
33 Der Apgar Score hat keinen direkten Wert für den Ablauf einer Neugeborenen Reanimation.

34 Der Heizstrahler ist wichtiger, als das Baby zu trocknen und einzuhüllen.

35 Ein notfallmäßiger venöser Zugang ist am besten über die Nabelvene zu bekommen.

36 Der Haupteffekt von Adrenalin ist die Vasodilatation.

37 Bronchial Lavage verbessert immer die Prognose bei Mekoniumaspiration.

38 Herzmassage ist sinnvoll, bevor die Lunge sich aufgebläht hat.

39 Die Atmung stoppt üblicherweise bevor der Kreislauf versagt.

40 Ein am Termin Geborenes kann mehr als $100 \mathrm{ml}$ Fruchtwasser ohne Hilfe beseitigen.

41 Ein Säugling mit einer Herzfrequenz unter 60/min muss sofort intubiert werden.

42 Thorax Kompressionen sollten den Thorax 1/3 des Weges bis zum Rücken eindrücken.

43 Systeme, die für eine Beatmung von Neugeborenen genutzt werden, sollten ein Überdruckventil von 30-40 cm Wassersäule besitzen.

44 Alle Kinder, die bei Geburt intubiert werden, müssen auf eine Neugeborenenintensivstation übernommen werden.

45 Hypothermie kann Apnoen verursachen.

46 Es ist ratsam die Reanimation eines Frühgeborenen mit einem niedrigerem Beatmungsdruck zu starten als bei einem reifen Neugeborenen.

47 Maskenbeatmung ist viel weniger effektiv als Beatmung nach Intubation.

48 Die initiale Reanimation eines Frühgeborenen unterscheidet sich sehr von einer Reanimation bei einem Reifgeborenen.

49 Schnappatmung ist immer flach.

50 Nach einer Reanimation ist wichtig: Abnahme von Nabelschnurblut und venösem Blut für $\mathrm{pH}$ und Base Excess Messungen. 
Anhang 7.4 Beurteilungsbogen nach dem Kurs 2008-2009

Deutscher Rat für Wiederbelebung im Kindesalter e.V.

German Resuszitation Concil e.V.

www.dr-wiki.de

\section{Ihr Feedback ist uns wichtig}

Um unsere Veranstaltungen so bedarfsorientiert wie möglich zu gestalten, möchten wir Ihre Meinung zu den Zielen und Inhalten, zur Kompetenz des Referenten und zur Organisation erfragen. Ferner haben Sie die Gelegenheit, uns Ihre persönlichen Bildungswünsche mitzuteilen. Bitte füllen Sie den Beurteilungsbogen bis zum Ende der Veranstaltung aus und geben inn an uns zurück.

Für Ihre Unterstützung bedanken wir uns sehr.

Thema: Reanimation des Neugeborenen Newborn Life Support NLS

Datum:[•]

Referent: Dr. U. Kreth \& Team

\begin{tabular}{|c|c|c|c|c|c|c|}
\hline Beurteilungskriterien & ++ & + & 0 & - & -- & Bemerkungen \\
\hline \multicolumn{7}{|l|}{ Ziele/Inhalte: } \\
\hline \multicolumn{7}{|l|}{$\begin{array}{l}\text { Wie beurteilen Sie den } \\
\text { Informationsaehalt? }\end{array}$} \\
\hline \multicolumn{7}{|l|}{$\begin{array}{l}\text { Wie schätzen Sie den praktischen } \\
\text { Nutzen für Ihre Arbeit ein? }\end{array}$} \\
\hline \multicolumn{7}{|l|}{$\begin{array}{l}\text { Hat das Seminar bzgl. Zielsetzung } \\
\text { und Inhalten Ihre Erwartungen } \\
\text { erfült? }\end{array}$} \\
\hline \multicolumn{7}{|l|}{ Referent: } \\
\hline \multicolumn{7}{|l|}{$\begin{array}{l}\text { Wie beurteilen Sie die } \\
\text { Fachkompetenz? }\end{array}$} \\
\hline \multicolumn{7}{|l|}{$\begin{array}{l}\text { Wie verständlich war die } \\
\text { Darstellung? }\end{array}$} \\
\hline \multicolumn{7}{|l|}{$\begin{array}{l}\text { Darstellung? } \\
\text { Wie beurteilen Sie die } \\
\text { kommunikative Kompetenz? }\end{array}$} \\
\hline \multicolumn{7}{|l|}{$\begin{array}{l}\text { Wie war die } \\
\text { Teilnehmerorientierung? }\end{array}$} \\
\hline \multicolumn{7}{|l|}{ Organisation: } \\
\hline \multicolumn{7}{|l|}{$\begin{array}{l}\text { War die Dauer der Veranstaltung } \\
\text { angemessen? }\end{array}$} \\
\hline \multicolumn{7}{|l|}{ Fazit: } \\
\hline $\begin{array}{l}\text { Wie beurteilen Sie das Seminar } \\
\text { insgesamt? }\end{array}$ & & & & & & \\
\hline
\end{tabular}

Was hat Ihnen besonders gut gefallen?

Was sollte Ihrer Meinung nach geändert werden?

Welche Fortbildungen wünschen Sie sich für Ihre weitere berufliche Entwicklung? 


\section{Anhang 7.5 Internet-Fragebogen 2008-2009}

Deutscher Rat für Wiederbelebung im Kindesalter e.V.

German Resuszitation Concil e.V. www.dr-wiki.de

\section{Newborn Life Support Kurs Internet-Fragebogen}

Bitte mit $R=$ richtig oder $F=$ falsch markieren.

1 Atmung stoppt nach 2-3 Minuten sobald das Gehirn keinen Sauerstoff mehr bekommt = primäre Apnoe.

2 Wenn Adrenalin gebraucht wird, ist die Situation oft schlecht.

3 Der Apgar Score hat keinen direkten Wert für den Ablauf einer Neugeborenen Reanimation.

4 Der Heizstrahler ist wichtiger, als das Baby zu trocknen und einzuhüllen.

5 Ein notfallmäßiger venöser Zugang ist am besten über die Nabelvene zu bekommen.

6 Der Haupteffekt von Adrenalin ist die Vasodilatation.

7 Bronchial Lavage verbessert immer die Prognose bei Mekoniumaspiration.

8 Herzmassage ist sinnvoll, bevor die Lunge sich aufgebläht hat.

9 Die Atmung stoppt üblicherweise bevor der Kreislauf versagt.

10 Ein am Termin Geborenes kann mehr als 100 ml Fruchtwasser ohne Hilfe beseitigen.

11 Ein Säugling mit einer Herzfrequenz unter 60/min muss sofort intubiert werden.

12 Thorax Kompressionen sollten den Thorax 1/3 des Weges bis zum Rücken eindrücken.

13 Systeme, die für eine Beatmung von Neugeborenen genutzt werden, sollten ein Überdruckventil von 30-40 cm Wassersäule besitzen.

14 Alle Kinder, die bei Geburt intubiert werden, müssen auf eine Neugeborenenintensivstation übernommen werden.

15 Hypothermie kann Apnoen verursachen.

Es ist ratsam die Reanimation eines Frühgeborenen mit einem niedrigerem Beatmungsdruck zu starten als bei einem reifen Neugeborenen.

16 Maskenbeatmung ist viel weniger effektiv als Beatmung nach Intubation.

17 Die initiale Reanimation eines Frühgeborenen unterscheidet sich sehr von einer Reanimation bei einem Reifgeborenen.

18 Schnappatmung ist immer flach.

19 Nach einer Reanimation ist wichtig: Abnahme von Nabelschnurblut und venösem Blut für $\mathrm{pH}$ und Base Excess Messungen. 
Anhang 7.6 Beispielhaftes Programm eines NLS-Kurses

AKH
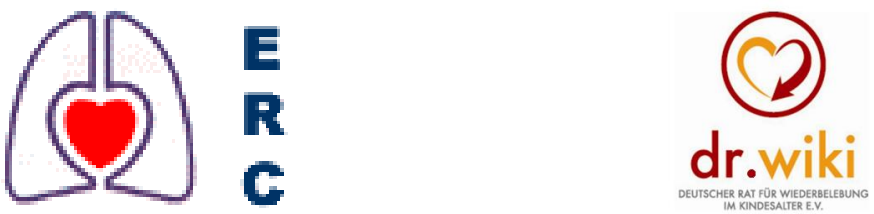

Kinderklinik St. Nikolaus Viersen Ausbildungszentrum Rheinland für pädiatrische Notfallmedizin

\section{Newborn \\ Life \\ Support}

Deutscher Rat für Wiederbelebung im Kindesalter e.V.

nach den Richtlinien des ERC European Resuscitation Council

$\begin{array}{ll}\text { [Datum }]- & {[\text { Ort }]} \\ & {[\text { Klinik }]}\end{array}$

\section{PROGRAMM}

Kursleitung:

$[1 \bullet]^{\star}$, ERC - Ausbilder

Internet: $\quad$ www.kinder-notfallkurse.de www.dr-wiki.de 
Deutscher Rat für Wiederbelebung im Kindesalter e.V.

Newborn Life Support Kurs

Viersen,

08.30 Uhr Faculty Meeting

08.45 Uhr Registrierung und Getränke

09.00 Uhr Vorstellung und Willkommen, Prätest

$[1 \bullet]^{*}$

09.15 Uhr Physiologie

$[2 \bullet]^{*}$

09.30 Uhr Reanimation bei Geburt

$[3 \bullet]^{*}$

$10.00 \mathrm{Uhr}$

Besondere Fälle

$[4 \bullet]^{\star}$

10.15 Uhr Pause

10.30 Uhr Skill Stations.

\begin{tabular}{||c|c|c|c|c|}
\hline Skill Stations & $\begin{array}{c}\text { Venöser } \\
\text { Zugang und } \\
\text { Medikamente }\end{array}$ & $\begin{array}{c}\text { Reanimations- } \\
\text { tisch \& } \\
\text { Kardiale } \\
\text { Reanimation }\end{array}$ & Atemwege 1 & Atemwege 2 \\
\hline \hline Ausbilder & $\begin{array}{c}{[2 \bullet]} \\
{[3 \bullet]}\end{array}$ & $\begin{array}{c}{[4 \bullet]} \\
{[5 \bullet]}\end{array}$ & $\begin{array}{c}{[6 \bullet]} \\
{[7 \bullet]}\end{array}$ & $\begin{array}{c}{[8 \bullet]} \\
{[9 \bullet]}\end{array}$ \\
\hline Raum & $\mathbf{1}$ & 2 & 3 & 4 \\
\hline $10.30-11.10$ & A & D & C & B \\
\hline $11.10-11.50$ & B & A & D & C \\
\hline $11.50-12.25$ & C & B & A & D \\
\hline $12.25-13.00$ & D & C & B & A \\
\hline
\end{tabular}

13.00

Mittagessen 
$14.00 \quad$ Demonstration

14.15 Szenarien

\begin{tabular}{|c|c|c|c|c|}
\hline Szenarien & Hausgeburt & $\begin{array}{l}\text { Früh- } \\
\text { geborene }\end{array}$ & Mekonium & $\begin{array}{l}\text { Anpassungs- } \\
\text { störung }\end{array}$ \\
\hline Ausbilder* & $\begin{array}{l}{[2 \bullet]} \\
{[3 \bullet]}\end{array}$ & $\begin{array}{l}{[4 \bullet]} \\
{[5 \bullet]}\end{array}$ & $\begin{array}{l}{[6 \bullet]} \\
{[7 \bullet]}\end{array}$ & $\begin{array}{l}{[8 \bullet]} \\
{[9 \bullet]}\end{array}$ \\
\hline Raum & 1 & 2 & 3 & 4 \\
\hline $14.15-14.45$ & $\mathbf{A}$ & D & C & $\boldsymbol{B}$ \\
\hline $14.45-15.15$ & B & $\mathbf{A}$ & D & C \\
\hline $15.15-15.45$ & C & B & $\mathbf{A}$ & D \\
\hline $15.45-16.15$ & D & $\underline{C}$ & B & $\mathbf{A}$ \\
\hline
\end{tabular}

$16.15 \quad$ Pause

16.30 Praktischer und schriftlicher Test

$17.45 \quad$ Faculty Meeting

18.00 Evaluation / Feedback / Ergebnisse

\section{Anmerkung:}

* [•] steht für den Namen eines Ausbilders, insgesamt werden 9 Ausbilder beschäftigt 
Anhang 7.7 Statistische Auswertung der Gesamtergebnisse

\section{Auswertung der Gesamtergebnisse}

\begin{tabular}{|ll|r|r|r|}
\hline & $\begin{array}{c}\text { Vor Schulung } \\
(\mathrm{t} 1)\end{array}$ & $\begin{array}{c}\text { Direkt nach } \\
\text { Schulung (t2) }\end{array}$ & $\begin{array}{c}\text { Nach 6 } \\
\text { Monaten (t3) }\end{array}$ \\
\hline \multicolumn{1}{|c|}{ Gültig } & 57 & 57 & 57 \\
Fehlend & 0 & 0 & 0 \\
Mittelwert (\%) & 81,42 & 92,67 & 86,89 \\
Median (\%) & 82,35 & 94,12 & 88,24 \\
Standardabweichung (\%) & 11,325 & 7,883 & 9,101 \\
Minimum (\%) & 47 & 59 & 59 \\
Maximum (\%) & $25(\%)$ & 100 & 100 & 100 \\
& 50 (\%) & 73,53 & 88,24 & 82,35 \\
Perzentile & 82,35 & 94,12 & 88,24 \\
& $75(\%)$ & 88,24 & 100,00 & 94,12 \\
\hline
\end{tabular}

Tabelle A1: Prozent Scores der Gesamtergebnisse von 57 Kandidaten

Vergleich der Prozent-Scores der Teilgruppe (57 Fälle) mit dem Wilcoxon Test

Vergleich der \%-Scores vor Schulung (t1) vs. direkt nach Schulung (t2)

\begin{tabular}{|l|r|}
\hline Wilcoxon-Test & $\begin{array}{c}\text { Direkt nach Schulung (t2) - } \\
\text { Vor Schulung (t1) }\end{array}$ \\
\hline$Z$ & $-6,022$ \\
Asymptotische Signifikanz & $<\mathbf{0 , 0 0 1}$ \\
\hline
\end{tabular}

Tabelle A2: Wilcoxon Test t1 vs t2

Vergleich der \%-Scores vor Schulung (t1) vs. nach 6 Monaten (t3)

\begin{tabular}{|l|r|}
\hline Wilcoxon-Test & $\begin{array}{c}\text { Nach 6 Monaten ( } \mathrm{t} 3)- \\
\text { Vor Schulung }(\mathrm{t} 1)\end{array}$ \\
\hline $\mathrm{Z}$ & $-3,921$ \\
$\begin{array}{l}\text { Asymptotische Signifikanz } \\
\text { (2-seitig) }\end{array}$ & $<0,001$ \\
\hline
\end{tabular}

Tabelle A3: Wilcoxon Test t1 vs $\mathrm{t} 3$ 
Vergleich der \%-Scores direkt nach Schulung (t2) vs. nach 6 Monaten (t3)

\begin{tabular}{|l|r|}
\hline Wilcoxon-Test & $\begin{array}{r}\text { Nach 6 Monaten (t3) - } \\
\text { Direkt nach Schulung (t2) }\end{array}$ \\
\hline$Z$ & $-4,076$ \\
Asymptotische Signifikanz & $<0,001$ \\
\hline (2-seitig) & \\
\hline
\end{tabular}

Tabelle A4: Wilcoxon Test t2 vs t3

Vergleich der Prozent-Scores der Gesamtgruppe (181 Fälle) mit dem Wilcoxon Test

\begin{tabular}{|l|c|c|}
\hline & $\begin{array}{c}\text { Vor dem Kurs } \\
(\mathbf{t} 1)\end{array}$ & $\begin{array}{c}\text { Direkt nach } \\
\text { dem Kurs (t2) }\end{array}$ \\
\hline $\mathrm{N}$ & 181 & 181 \\
Mittelwert (\%) & 80,99 & 92,33 \\
Median (\%) & 82,35 & 94,12 \\
Standardabweichung (\%) & 10,543 & 8,299 \\
\hline
\end{tabular}

Tabelle A5: Prozent-Scores t1 vs. t2

\begin{tabular}{|l|r|}
\hline Wilcoxon-Test & $\begin{array}{r}\text { Direkt nach } \\
\text { Schulung (t2) - } \\
\text { Vor Schulung (t1) }\end{array}$ \\
\hline$Z$ & $-10,433$ \\
Asymptotische Signifikanz \\
(2-seitig)
\end{tabular}

Tabelle A6: Wilcoxon Test t1 vs. t2 


\section{Anhang 7.8 Vergleich der einzelnen Fragen zu drei Zeitpunkten}

\section{Vergleich der Richtigen Antworten der Teilgruppe (57 Fälle) zu den drei Zeitpunkten}

a) Vergleich vor Schulung (t1) vs. direkt nach Schulung (t2) (57 Fälle)

1) Der Heizstrahler ist wichtiger, als das Baby zu trocknen und einzuhüllen

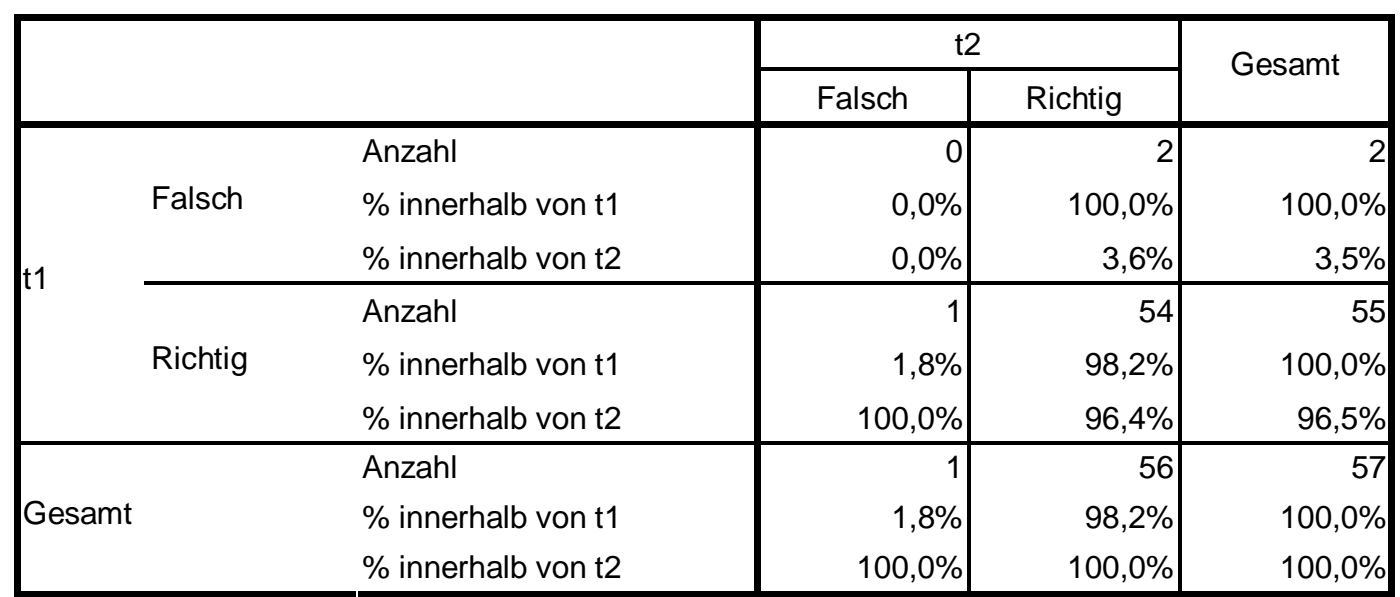

Tabelle A7: Der Heizstrahler ist wichtiger, als das Baby zu trocknen und einzuhüllen t1 vs. t2

\begin{tabular}{|l|r|c|}
\hline & Wert & $\begin{array}{c}\text { Exakte Signifikanz } \\
\text { (2-seitig) }\end{array}$ \\
\hline $\begin{array}{l}\text { McNemar-Test } \\
\text { Anzahl der gültigen Fälle }\end{array}$ & 57 & $\mathbf{1 , 0 0 0}$ \\
\hline
\end{tabular}

Tabelle A8: McNemar-Test

2) Ein notfallmäßiger venöser Zugang ist am besten über die Nabelvene zu bekommen

\begin{tabular}{|c|c|c|c|c|c|}
\hline & & & \multicolumn{2}{|c|}{ t2 } & \multirow{2}{*}{ Gesamt } \\
\hline & & & Falsch & Richtig & \\
\hline \multirow{6}{*}{ t1 } & \multirow{3}{*}{ Falsch } & Anzahl & 1 & 4 & 5 \\
\hline & & $\%$ innerhalb von $t 1$ & $20,0 \%$ & $80,0 \%$ & $100,0 \%$ \\
\hline & & $\%$ innerhalb von $t 2$ & $100,0 \%$ & $7,1 \%$ & $8,8 \%$ \\
\hline & \multirow{3}{*}{ Richtig } & Anzahl & 0 & 52 & 52 \\
\hline & & $\%$ innerhalb von $t 1$ & $0,0 \%$ & $100,0 \%$ & $100,0 \%$ \\
\hline & & $\%$ innerhalb von t2 & $0,0 \%$ & $92,9 \%$ & $91,2 \%$ \\
\hline \multirow{3}{*}{\multicolumn{2}{|c|}{ Gesamt }} & Anzahl & 1 & 56 & 57 \\
\hline & & $\%$ innerhalb von $t 1$ & $1,8 \%$ & $98,2 \%$ & $100,0 \%$ \\
\hline & & $\%$ innerhalb von t2 & $100,0 \%$ & $100,0 \%$ & $100,0 \%$ \\
\hline
\end{tabular}

Tabelle A9:Ein notfallmäßiger venöser Zugang ist am besten über die Nabelvene zu bekommen t1 vs. t2

\begin{tabular}{|l|r|c|}
\hline & Wert & $\begin{array}{c}\text { Exakte Signifikanz } \\
\text { (2-seitig) }\end{array}$ \\
\hline $\begin{array}{l}\text { McNemar-Test } \\
\text { Anzahl der gültigen Fälle }\end{array}$ & 57 & $\mathbf{0 , 1 2 5}$ \\
\hline
\end{tabular}




\begin{tabular}{|l|r|c|}
\hline & Wert & $\begin{array}{c}\text { Exakte Signifikanz } \\
\text { (2-seitig) }\end{array}$ \\
\hline $\begin{array}{l}\text { McNemar-Test } \\
\text { Anzahl der gültigen Fälle }\end{array}$ & 57 & $\mathbf{0 , 1 2 5}$ \\
\hline
\end{tabular}

Tabelle A10: McNemar-Test

\section{3) Der Haupteffekt von Adrenalin ist die Vasodilatation}

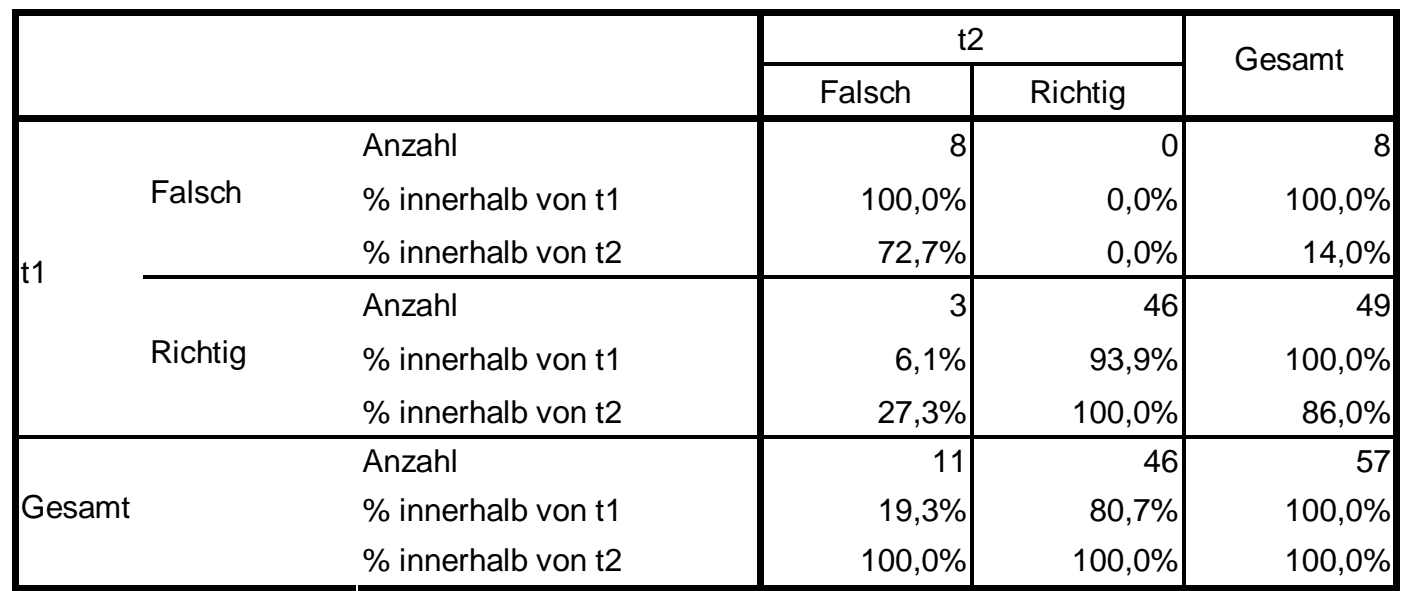

Tabelle A11: Der Haupteffekt von Adrenalin ist die Vasodilatation t1 vs. t2

\begin{tabular}{|l|r|c|}
\hline & Wert & $\begin{array}{c}\text { Exakte Signifikanz } \\
\text { (2-seitig) }\end{array}$ \\
\hline $\begin{array}{l}\text { McNemar-Test } \\
\text { Anzahl der gültigen Fälle }\end{array}$ & 57 & $\mathbf{0 , 2 5 0}$ \\
\hline
\end{tabular}

Tabelle A12: McNemar-Test

\section{4) Bronchiallavage verbessert immer die Prognose bei Mekoniumaspiration}

\begin{tabular}{|c|c|c|c|c|c|}
\hline & & & \multicolumn{2}{|c|}{ t2 } & \multirow{2}{*}{ Gesamt } \\
\hline & & & Falsch & Richtig & \\
\hline \multirow{6}{*}{$\mathrm{t} 1$} & \multirow{3}{*}{ Falsch } & Anzahl & 11 & 14 & 25 \\
\hline & & $\%$ innerhalb von $\mathrm{t} 1$ & $44,0 \%$ & $56,0 \%$ & $100,0 \%$ \\
\hline & & $\%$ innerhalb von t2 & $84,6 \%$ & $31,8 \%$ & $43,9 \%$ \\
\hline & \multirow{3}{*}{ Richtig } & Anzahl & 2 & 30 & 32 \\
\hline & & $\%$ innerhalb von $\mathrm{t} 1$ & $6,3 \%$ & $93,8 \%$ & $100,0 \%$ \\
\hline & & $\%$ innerhalb von t2 & $15,4 \%$ & $68,2 \%$ & $56,1 \%$ \\
\hline \multirow{3}{*}{\multicolumn{2}{|c|}{ Gesamt }} & Anzahl & 13 & 44 & 57 \\
\hline & & $\%$ innerhalb von $\mathrm{t} 1$ & $22,8 \%$ & $77,2 \%$ & $100,0 \%$ \\
\hline & & $\%$ innerhalb von t2 & $100,0 \%$ & $100,0 \%$ & $100,0 \%$ \\
\hline
\end{tabular}

Tabelle A13: Bronchiallavage verbessert immer die Prognose bei Mekoniumaspiration t1 vs. t2

\begin{tabular}{|l|r|c|}
\hline & Wert & $\begin{array}{c}\text { Exakte Signifikanz } \\
\text { (2-seitig) }\end{array}$ \\
\hline $\begin{array}{l}\text { McNemar-Test } \\
\text { Anzahl der gültigen Fälle }\end{array}$ & 57 & $\mathbf{0 , 0 0 4}$ \\
\hline
\end{tabular}

Tabelle A14: McNemar-Test 
5) Herzmassage ist sinnvoll, bevor die Lunge sich aufgebläht hat

\begin{tabular}{|c|c|c|c|c|}
\hline & & & $\mathrm{t} 2$ & \multirow{2}{*}{ Gesamt } \\
\hline & & & Richtig & \\
\hline \multirow{6}{*}{$\mathrm{t} 1$} & \multirow{3}{*}{ Falsch } & Anzahl & 6 & 6 \\
\hline & & $\%$ innerhalb von $\mathrm{t} 1$ & $100,0 \%$ & $100,0 \%$ \\
\hline & & $\%$ innerhalb von $\mathrm{t} 2$ & $10,5 \%$ & $10,5 \%$ \\
\hline & \multirow{3}{*}{ Richtig } & Anzahl & 51 & 51 \\
\hline & & $\%$ innerhalb von $\mathrm{t} 1$ & $100,0 \%$ & $100,0 \%$ \\
\hline & & $\%$ innerhalb von $\mathrm{t} 2$ & $89,5 \%$ & $89,5 \%$ \\
\hline \multirow{3}{*}{\multicolumn{2}{|c|}{ Gesamt }} & Anzahl & 57 & 57 \\
\hline & & $\%$ innerhalb von $\mathrm{t} 1$ & $100,0 \%$ & $100,0 \%$ \\
\hline & & $\%$ innerhalb von $\mathrm{t} 2$ & $100,0 \%$ & $100,0 \%$ \\
\hline
\end{tabular}

Tabelle A 15:Herzmassage ist sinnvoll, bevor die Lunge sich aufgebläht hat t1 vs. t2

6) Die Atmung stoppt üblicherweise, bevor der Kreislauf versagt

\begin{tabular}{|c|c|c|c|c|c|}
\hline & & & \multicolumn{2}{|c|}{ t2 } & \multirow{2}{*}{ Gesamt } \\
\hline & & & Falsch & Richtig & \\
\hline \multirow{6}{*}{$t 1$} & \multirow{3}{*}{ Falsch } & Anzahl & 4 & 11 & 15 \\
\hline & & $\%$ innerhalb von $\mathrm{t} 1$ & $26,7 \%$ & $73,3 \%$ & $100,0 \%$ \\
\hline & & $\%$ innerhalb von t2 & $80,0 \%$ & $21,2 \%$ & $26,3 \%$ \\
\hline & \multirow{3}{*}{ Richtig } & Anzahl & 1 & 41 & 42 \\
\hline & & $\%$ innerhalb von $\mathrm{t} 1$ & $2,4 \%$ & $97,6 \%$ & $100,0 \%$ \\
\hline & & $\%$ innerhalb von t2 & $20,0 \%$ & $78,8 \%$ & $73,7 \%$ \\
\hline \multirow{3}{*}{\multicolumn{2}{|c|}{ Gesamt }} & Anzahl & 5 & 52 & 57 \\
\hline & & $\%$ innerhalb von $\mathrm{t} 1$ & $8,8 \%$ & $91,2 \%$ & $100,0 \%$ \\
\hline & & $\%$ innerhalb von t2 & $100,0 \%$ & $100,0 \%$ & $100,0 \%$ \\
\hline
\end{tabular}

Tabelle A16: Die Atmung stoppt üblicherweise, bevor der Kreislauf versagt t1 vs. t2

\begin{tabular}{|l|r|c|}
\hline & Wert & $\begin{array}{c}\text { Exakte Signifikanz } \\
\text { (2-seitig) }\end{array}$ \\
\hline $\begin{array}{l}\text { McNemar-Test } \\
\text { Anzahl der gültigen Fälle }\end{array}$ & 57 & $\mathbf{0 , 0 0 6}$ \\
\hline
\end{tabular}

Tabelle A17: McNemar-Test

7) Ein am Termin Geborenes kann mehr als $100 \mathrm{ml}$ Fruchtwasser ohne Hilfe beseitigen

\begin{tabular}{|c|c|c|c|c|c|}
\hline & & & \multicolumn{2}{|c|}{ t2 } & \multirow{2}{*}{ Gesamt } \\
\hline & & & Falsch & Richtig & \\
\hline \multirow{6}{*}{$\mathrm{t} 1$} & \multirow{3}{*}{ Falsch } & Anzahl & 4 & 17 & 21 \\
\hline & & $\%$ innerhalb von $\mathrm{t} 1$ & $19,0 \%$ & $81,0 \%$ & $100,0 \%$ \\
\hline & & $\%$ innerhalb von t2 & $50,0 \%$ & $34,7 \%$ & $36,8 \%$ \\
\hline & \multirow{3}{*}{ Richtig } & Anzahl & 4 & 32 & 36 \\
\hline & & $\%$ innerhalb von $\mathrm{t} 1$ & $11,1 \%$ & $88,9 \%$ & $100,0 \%$ \\
\hline & & $\%$ innerhalb von t2 & $50,0 \%$ & $65,3 \%$ & $63,2 \%$ \\
\hline \multirow{3}{*}{\multicolumn{2}{|c|}{ Gesamt }} & Anzahl & 8 & 49 & 57 \\
\hline & & $\%$ innerhalb von $\mathrm{t} 1$ & $14,0 \%$ & $86,0 \%$ & $100,0 \%$ \\
\hline & & $\%$ innerhalb von t2 & $100,0 \%$ & $100,0 \%$ & $100,0 \%$ \\
\hline
\end{tabular}

Tabelle A18: Ein am Termin Geborenes kann mehr als 100 ml Fruchtwasser ohne Hilfe beseitigen t1 vs. t2 


\begin{tabular}{|l|r|c|}
\hline & Wert & $\begin{array}{c}\text { Exakte Signifikanz } \\
\text { (2-seitig) }\end{array}$ \\
\hline $\begin{array}{l}\text { McNemar-Test } \\
\text { Anzahl der gültigen Fälle }\end{array}$ & 57 & $\mathbf{0 , 0 0 7}$ \\
\hline
\end{tabular}

Tabelle A19: McNemar-Test

8) Ein Säugling mit Herzfrequenz unter 60/min muss sofort intubiert werden

\begin{tabular}{|c|c|c|c|c|c|}
\hline & & & \multicolumn{2}{|c|}{ t2 } & \multirow{2}{*}{ Gesamt } \\
\hline & & & Falsch & Richtig & \\
\hline \multirow{6}{*}{$\mathrm{t} 1$} & \multirow{3}{*}{ Falsch } & Anzahl & 2 & 7 & 9 \\
\hline & & $\%$ innerhalb von $t 1$ & $22,2 \%$ & $77,8 \%$ & $100,0 \%$ \\
\hline & & $\%$ innerhalb von $\mathrm{t} 2$ & $100,0 \%$ & $12,7 \%$ & $15,8 \%$ \\
\hline & \multirow{3}{*}{ Richtig } & Anzahl & 0 & 48 & 48 \\
\hline & & $\%$ innerhalb von $\mathrm{t} 1$ & $0,0 \%$ & $100,0 \%$ & $100,0 \%$ \\
\hline & & $\%$ innerhalb von t2 & $0,0 \%$ & $87,3 \%$ & $84,2 \%$ \\
\hline \multirow{3}{*}{\multicolumn{2}{|c|}{ Gesamt }} & Anzahl & 2 & 55 & 57 \\
\hline & & $\%$ innerhalb von t1 & $3,5 \%$ & $96,5 \%$ & $100,0 \%$ \\
\hline & & $\%$ innerhalb von t2 & $100,0 \%$ & $100,0 \%$ & $100,0 \%$ \\
\hline
\end{tabular}

Tabelle A20: Ein Säugling mit Herzfrequenz unter 60/min muss sofort intubiert werden t1 vs. t2

\begin{tabular}{|l|r|c|}
\hline & Wert & $\begin{array}{c}\text { Exakte Signifikanz } \\
\text { (2-seitig) }\end{array}$ \\
\hline $\begin{array}{l}\text { McNemar-Test } \\
\text { Anzahl der gültigen Fälle }\end{array}$ & 57 & $\mathbf{0 , 0 1 6}$ \\
\hline
\end{tabular}

Tabelle A21: McNemar-Test

9) Thoraxkompressionen sollten den Thorax 1/3 des Weges bis zum Rücken eindrücken

\begin{tabular}{|c|c|c|c|c|c|}
\hline & & & \multicolumn{2}{|c|}{ t2 } & \multirow{2}{*}{ Gesamt } \\
\hline & & & Falsch & Richtig & \\
\hline \multirow{6}{*}{ t1 } & \multirow{3}{*}{ Falsch } & Anzahl & 0 & 6 & 6 \\
\hline & & $\%$ innerhalb von $\mathrm{t} 1$ & $0,0 \%$ & $100,0 \%$ & $100,0 \%$ \\
\hline & & $\%$ innerhalb von t2 & $0,0 \%$ & $10,7 \%$ & $10,5 \%$ \\
\hline & \multirow{3}{*}{ Richtig } & Anzahl & 1 & 50 & 51 \\
\hline & & $\%$ innerhalb von $\mathrm{t} 1$ & $2,0 \%$ & $98,0 \%$ & $100,0 \%$ \\
\hline & & $\%$ innerhalb von t2 & $100,0 \%$ & $89,3 \%$ & $89,5 \%$ \\
\hline \multirow{3}{*}{\multicolumn{2}{|c|}{ Gesamt }} & Anzahl & 1 & 56 & 57 \\
\hline & & $\%$ innerhalb von $\mathrm{t} 1$ & $1,8 \%$ & $98,2 \%$ & $100,0 \%$ \\
\hline & & $\%$ innerhalb von t2 & $100,0 \%$ & $100,0 \%$ & $100,0 \%$ \\
\hline
\end{tabular}

Tabelle A22: Thoraxkompressionen sollten den Thorax 1/3 des Weges bis zum Rücken eindrücken t1 vs. t2

\begin{tabular}{|l|r|c|}
\hline & Wert & $\begin{array}{c}\text { Exakte Signifikanz } \\
\text { (2-seitig) }\end{array}$ \\
\hline $\begin{array}{l}\text { McNemar-Test } \\
\text { Anzahl der gültigen Fälle }\end{array}$ & 57 & $\mathbf{0 , 1 2 5}$ \\
\hline
\end{tabular}

Tabelle A23: McNemar-Test 
10) Systeme, die zur Beatmung von Neugeborenen benutzt werden, sollten ein Überdruckventil von 30-40 cm Wassersäule besitzen

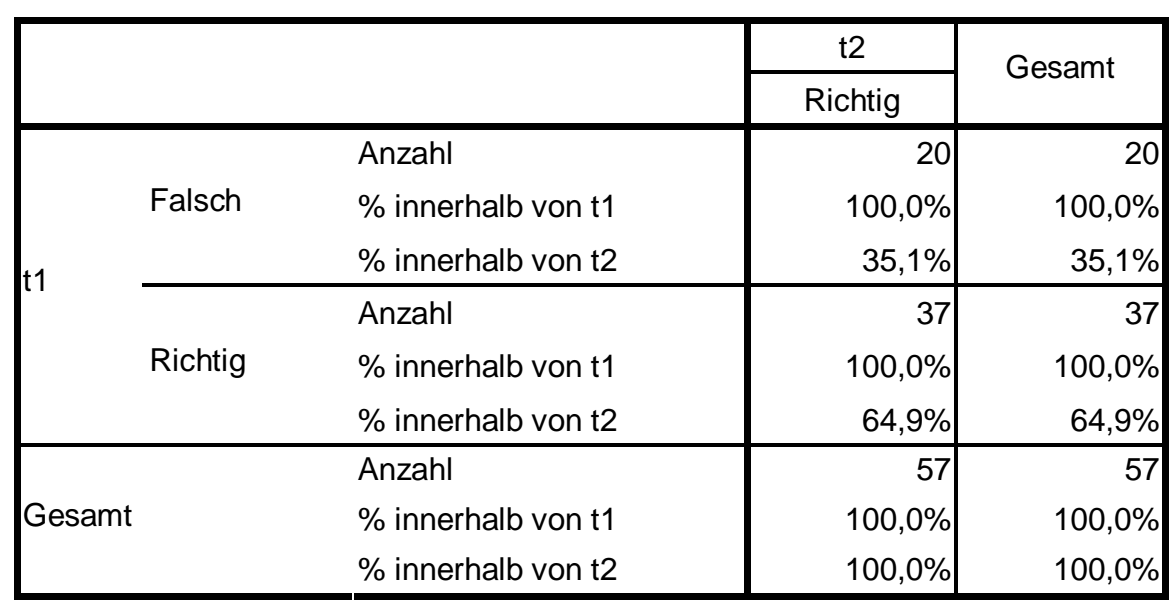

Tabelle A24: Systeme, die zur Beatmung von Neugeborenen benutzt werden, sollten ein Überdruckventil von 30 $40 \mathrm{~cm}$ Wassersäule besitzen t1 vs. $\mathrm{t} 2$

11) Alle Kinder, die bei Geburt intubiert werden, müssen auf eine Neugeborenenintensivstation übernommen werden

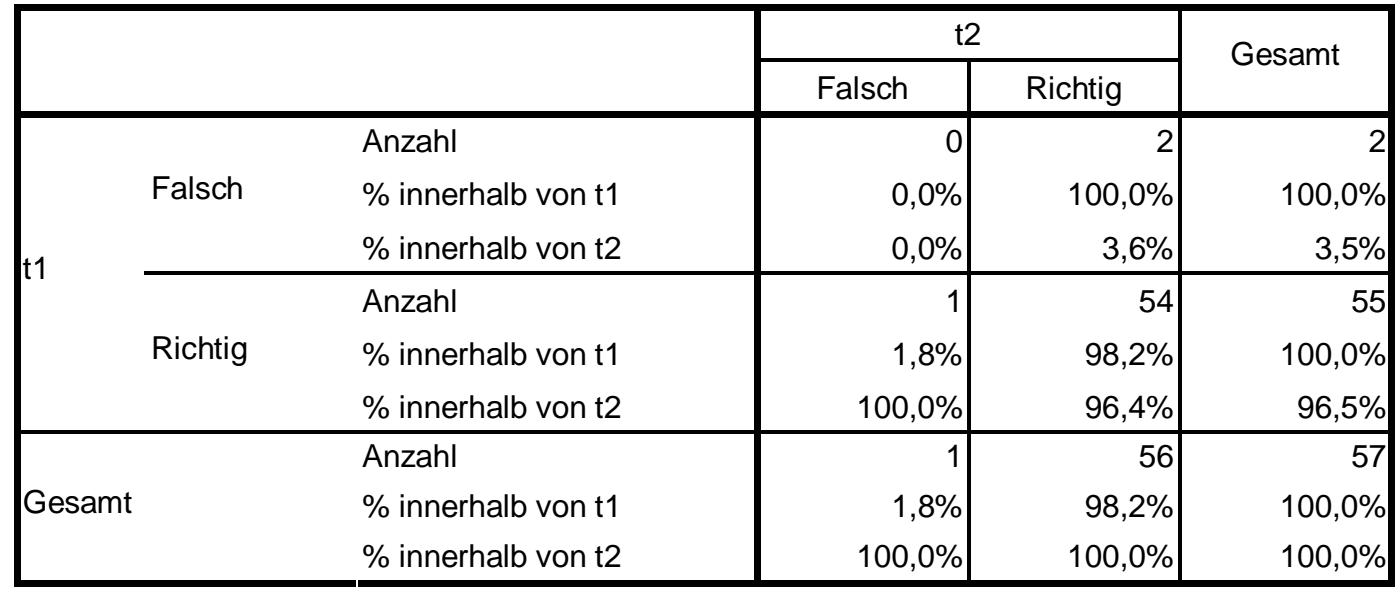

Tabelle A25: Alle Kinder, die bei Geburt intubiert werden, müssen auf eine Neugeborenenintensivstation übernommen werden $\mathrm{t} 1$ vs. t2

\begin{tabular}{|l|r|c|}
\hline & Wert & $\begin{array}{c}\text { Exakte Signifikanz } \\
\text { (2-seitig) }\end{array}$ \\
\hline $\begin{array}{l}\text { McNemar-Test } \\
\text { Anzahl der gültigen Fälle }\end{array}$ & 57 & $\mathbf{1 , 0 0 0}$ \\
\hline
\end{tabular}

Tabelle A26: McNemar-Test 
12)

Hypothermie kann Apnoen verursachen

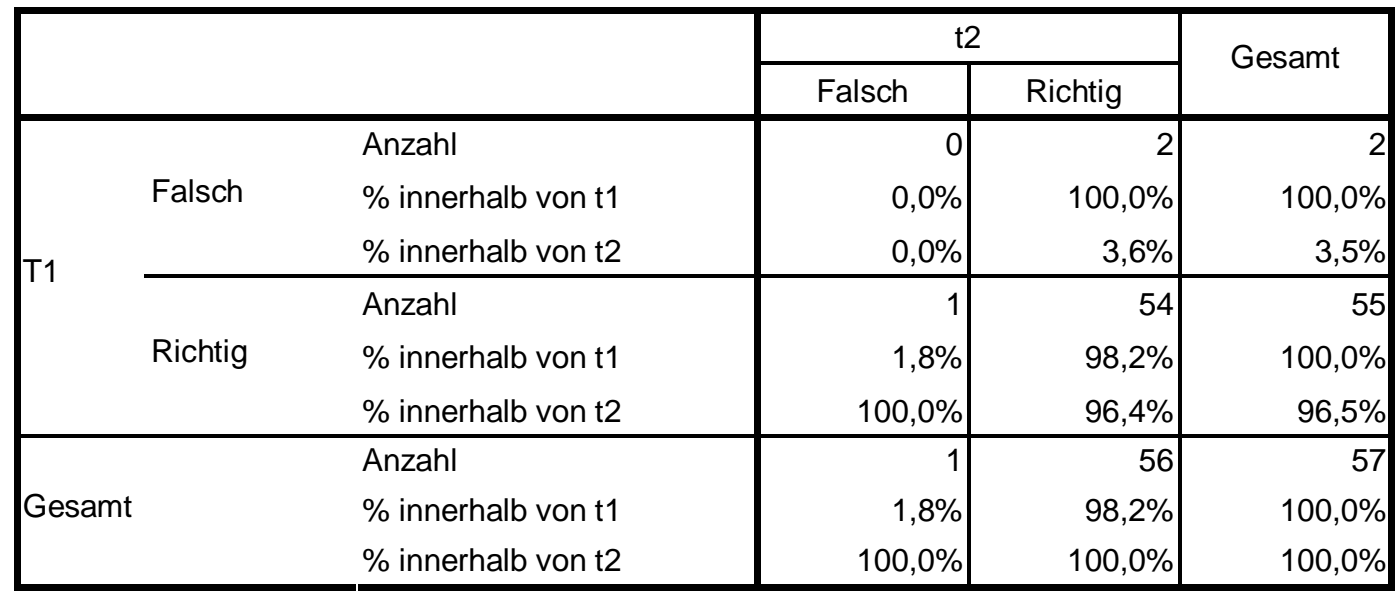

Tabelle A27: Hypothermie kann Apnoen verursachen t1 vs. t2

\begin{tabular}{|l|r|c|}
\hline & Wert & $\begin{array}{c}\text { Exakte Signifikanz } \\
\text { (2-seitig) }\end{array}$ \\
\hline $\begin{array}{l}\text { McNemar-Test } \\
\text { Anzahl der gültigen Fälle }\end{array}$ & 57 & $\mathbf{1 , 0 0 0}$ \\
\hline
\end{tabular}

Tabelle A28: McNemar-Test

13) Es ist ratsam, die Reanimation bei einem Frühgeborenen mit einem niedrigeren Beatmungsdruck zu starten als bei einem reifen Neugeborenen

\begin{tabular}{|c|c|c|c|c|c|}
\hline & & & \multicolumn{2}{|c|}{ t2 } & \multirow{2}{*}{ Gesamt } \\
\hline & & & Falsch & Richtig & \\
\hline \multirow{6}{*}{ t1 } & \multirow{3}{*}{ Falsch } & Anzahl & 2 & 11 & 13 \\
\hline & & $\%$ innerhalb von $t 1$ & $15,4 \%$ & $84,6 \%$ & $100,0 \%$ \\
\hline & & $\%$ innerhalb von $\mathrm{t} 2$ & $66,7 \%$ & $20,4 \%$ & $22,8 \%$ \\
\hline & \multirow{3}{*}{ Richtig } & Anzahl & 1 & 43 & 44 \\
\hline & & $\%$ innerhalb von $\mathrm{t} 1$ & $2,3 \%$ & $97,7 \%$ & $100,0 \%$ \\
\hline & & $\%$ innerhalb von $\mathrm{t} 2$ & $33,3 \%$ & $79,6 \%$ & $77,2 \%$ \\
\hline \multirow{3}{*}{\multicolumn{2}{|c|}{ Gesamt }} & Anzahl & 3 & 54 & 57 \\
\hline & & $\%$ innerhalb von $\mathrm{t} 1$ & $5,3 \%$ & $94,7 \%$ & $100,0 \%$ \\
\hline & & $\%$ innerhalb von $\mathrm{t} 2$ & $100,0 \%$ & $100,0 \%$ & $100,0 \%$ \\
\hline
\end{tabular}

Tabelle A 29: Es ist ratsam, die Reanimation bei einem Frühgeborenen mit einem niedrigeren Beatmungsdruck zu starten als bei einem reifen Neugeborenen t1 vs. t2

\begin{tabular}{|l|c|c|}
\hline & Wert & $\begin{array}{c}\text { Exakte Signifikanz } \\
\text { (2-seitig) }\end{array}$ \\
\hline $\begin{array}{l}\text { McNemar-Test } \\
\text { Anzahl der gültigen Fälle }\end{array}$ & 57 & $\mathbf{0 , 0 0 6}$ \\
\hline
\end{tabular}

Tabelle A30: McNemar-Test 
14) Maskenbeatmung ist viel weniger effektiv als Beatmung nach Intubation

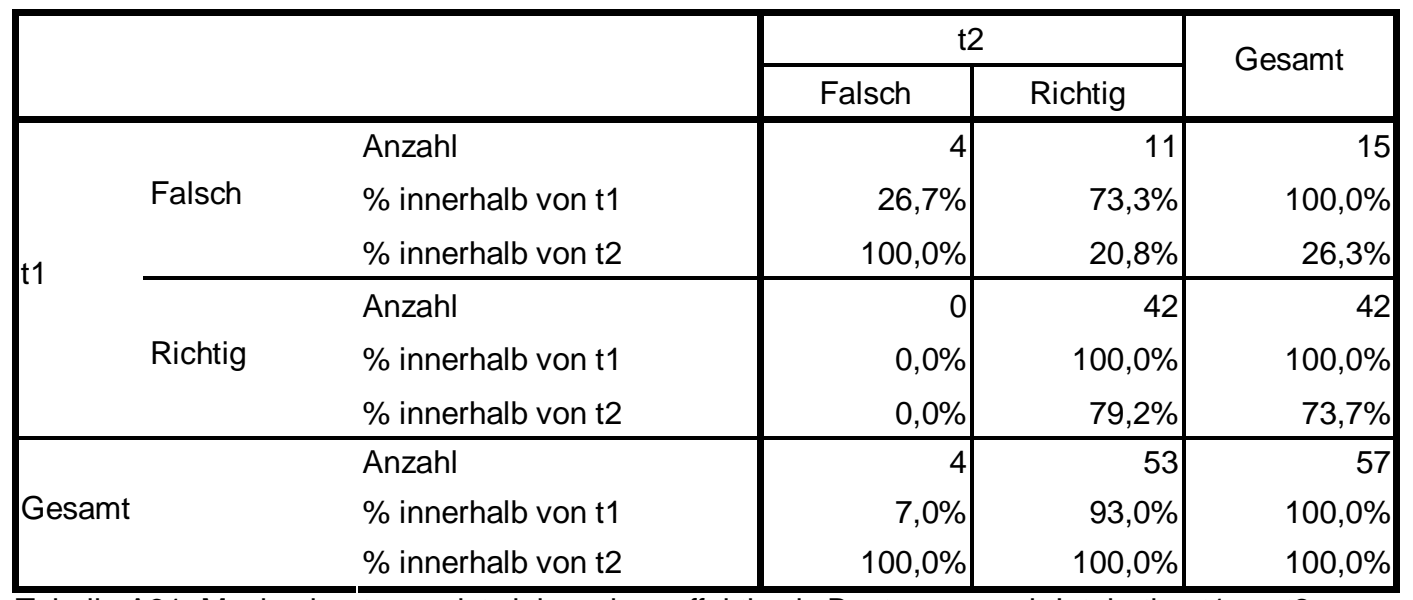

Tabelle A31: Maskenbeatmung ist viel weniger effektiv als Beatmung nach Intubation t1 vs. t2

\begin{tabular}{|l|r|c|}
\hline & Wert & $\begin{array}{c}\text { Exakte Signifikanz } \\
\text { (2-seitig) }\end{array}$ \\
\hline $\begin{array}{l}\text { McNemar-Test } \\
\text { Anzahl der gültigen Fälle }\end{array}$ & 57 & $\mathbf{0 , 0 0 1}$ \\
\hline
\end{tabular}

Tabelle A32: McNemar-Test

15) Die initiale Reanimation eines Frühgeborenen unterscheidet sich sehr von einer Reanimation bei einem Reifgeborenen

\begin{tabular}{|c|c|c|c|c|c|}
\hline & & & \multicolumn{2}{|c|}{ t2 } & \multirow{2}{*}{ Gesamt } \\
\hline & & & Falsch & Richtig & \\
\hline \multirow{6}{*}{$\mathrm{t} 1$} & \multirow{3}{*}{ Falsch } & Anzahl & 0 & 16 & 16 \\
\hline & & $\%$ innerhalb von $\mathrm{t} 1$ & $0,0 \%$ & $100,0 \%$ & $100,0 \%$ \\
\hline & & $\%$ innerhalb von t2 & $0,0 \%$ & $30,2 \%$ & $28,1 \%$ \\
\hline & \multirow{3}{*}{ Richtig } & Anzahl & 4 & 37 & 41 \\
\hline & & $\%$ innerhalb von $\mathrm{t} 1$ & $9,8 \%$ & $90,2 \%$ & $100,0 \%$ \\
\hline & & $\%$ innerhalb von t2 & $100,0 \%$ & $69,8 \%$ & $71,9 \%$ \\
\hline \multirow{3}{*}{\multicolumn{2}{|c|}{ Gesamt }} & Anzahl & 4 & 53 & 57 \\
\hline & & $\%$ innerhalb von $\mathrm{t} 1$ & $7,0 \%$ & $93,0 \%$ & $100,0 \%$ \\
\hline & & $\%$ innerhalb von t2 & $100,0 \%$ & $100,0 \%$ & $100,0 \%$ \\
\hline
\end{tabular}

Tabelle A33: Die initiale Reanimation eines Frühgeborenen unterscheidet sich sehr von einer Reanimation bei einem Reifgeborenen $\mathrm{t} 1$ vs. t2

\begin{tabular}{|l|r|c|}
\hline & Wert & $\begin{array}{c}\text { Exakte Signifikanz } \\
\text { (2-seitig) }\end{array}$ \\
\hline $\begin{array}{l}\text { McNemar-Test } \\
\text { Anzahl der gültigen Fälle }\end{array}$ & 57 & $\mathbf{0 , 0 1 2}$ \\
\hline
\end{tabular}

Tabelle A34: McNemar-Test 
16)

Schnappatmung ist immer flach

\begin{tabular}{|c|c|c|c|c|c|}
\hline & & & \multicolumn{2}{|c|}{$\mathrm{t} 2$} & \multirow{2}{*}{ Gesamt } \\
\hline & & & Falsch & Richtig & \\
\hline \multirow{6}{*}{$t 1$} & \multirow{3}{*}{ Falsch } & Anzahl & 6 & 5 & 11 \\
\hline & & $\%$ innerhalb von $\mathrm{t} 1$ & $54,5 \%$ & $45,5 \%$ & $100,0 \%$ \\
\hline & & $\%$ innerhalb von t2 & $46,2 \%$ & $11,4 \%$ & $19,3 \%$ \\
\hline & \multirow{3}{*}{ Richtig } & Anzahl & 7 & 39 & 46 \\
\hline & & $\%$ innerhalb von $\mathrm{t} 1$ & $15,2 \%$ & $84,8 \%$ & $100,0 \%$ \\
\hline & & $\%$ innerhalb von $\mathrm{t} 2$ & $53,8 \%$ & $88,6 \%$ & $80,7 \%$ \\
\hline \multirow{3}{*}{\multicolumn{2}{|c|}{ Gesamt }} & Anzahl & 13 & 44 & 57 \\
\hline & & $\%$ innerhalb von t1 & $22,8 \%$ & $77,2 \%$ & $100,0 \%$ \\
\hline & & $\%$ innerhalb von t2 & $100,0 \%$ & $100,0 \%$ & $100,0 \%$ \\
\hline
\end{tabular}

Tabelle A35: Schnappatmung ist immer flach t1 vs. t2

\begin{tabular}{|l|r|c|}
\hline & Wert & $\begin{array}{c}\text { Exakte Signifikanz } \\
\text { (2-seitig) }\end{array}$ \\
\hline $\begin{array}{l}\text { McNemar-Test } \\
\text { Anzahl der gültigen Fälle }\end{array}$ & 57 & $\mathbf{0 , 7 7 4}$ \\
\hline
\end{tabular}

Tabelle A36: McNemar-Test

17) Nach einer Reanimation ist wichtig: Abnahme von Nabelschnurblut und venösem Blut zur Messung von $\mathrm{pH}$ und $\mathrm{BE}$

\begin{tabular}{|c|c|c|c|c|c|}
\hline & & & \multicolumn{2}{|c|}{ t2 } & \multirow{2}{*}{ Gesamt } \\
\hline & & & Falsch & Richtig & \\
\hline \multirow{6}{*}{$\mathrm{t} 1$} & \multirow{3}{*}{ Falsch } & Anzahl & 1 & 3 & 4 \\
\hline & & $\%$ innerhalb von $\mathrm{t} 1$ & $25,0 \%$ & $75,0 \%$ & $100,0 \%$ \\
\hline & & $\%$ innerhalb von t2 & $33,3 \%$ & $5,6 \%$ & $7,0 \%$ \\
\hline & \multirow{3}{*}{ Richtig } & Anzahl & 2 & 51 & 53 \\
\hline & & $\%$ innerhalb von $\mathrm{t} 1$ & $3,8 \%$ & $96,2 \%$ & $100,0 \%$ \\
\hline & & $\%$ innerhalb von $t 2$ & $66,7 \%$ & $94,4 \%$ & $93,0 \%$ \\
\hline \multirow{3}{*}{\multicolumn{2}{|c|}{ Gesamt }} & Anzahl & 3 & 54 & 57 \\
\hline & & $\%$ innerhalb von $\mathrm{t} 1$ & $5,3 \%$ & $94,7 \%$ & $100,0 \%$ \\
\hline & & $\%$ innerhalb von t2 & $100,0 \%$ & $100,0 \%$ & $100,0 \%$ \\
\hline
\end{tabular}

Tabelle A37: Nach einer Reanimation ist wichtig: Abnahme von Nabelschnurblut und venösem Blut zur Messung von $\mathrm{pH}$ und $\mathrm{BE}$ t1 vs. $\mathrm{t} 2$

\begin{tabular}{|l|r|c|}
\hline & Wert & $\begin{array}{c}\text { Exakte Signifikanz } \\
\text { (2-seitig) }\end{array}$ \\
\hline $\begin{array}{l}\text { McNemar-Test } \\
\text { Anzahl der gültigen Fälle }\end{array}$ & 57 & $\mathbf{1 , 0 0 0}$ \\
\hline
\end{tabular}

Tabelle A38: McNemar-Test 
b) Vergleich vor Schulung (t1) vs. nach 6 Monaten (t3) (57 Fälle)

1) Der Heizstrahler ist wichtiger als das Baby zu trocknen und einzuhüllen

\begin{tabular}{|c|c|c|c|c|}
\hline & & & t3 & \multirow{2}{*}{ Gesamt } \\
\hline & & & Richtig & \\
\hline \multirow{6}{*}{$\mathrm{t} 1$} & \multirow{3}{*}{ Falsch } & Anzahl & 2 & 2 \\
\hline & & $\%$ innerhalb von $\mathrm{t} 1$ & $100,0 \%$ & $100,0 \%$ \\
\hline & & $\%$ innerhalb von t3 & $3,5 \%$ & $3,5 \%$ \\
\hline & \multirow{3}{*}{ Richtig } & Anzahl & 55 & 55 \\
\hline & & $\%$ innerhalb von $\mathrm{t} 1$ & $100,0 \%$ & $100,0 \%$ \\
\hline & & $\%$ innerhalb von t3 & $96,5 \%$ & $96,5 \%$ \\
\hline \multirow{3}{*}{\multicolumn{2}{|c|}{ Gesamt }} & Anzahl & 57 & 57 \\
\hline & & $\%$ innerhalb von $\mathrm{t} 1$ & $100,0 \%$ & $100,0 \%$ \\
\hline & & $\%$ innerhalb von t3 & $100,0 \%$ & $100,0 \%$ \\
\hline
\end{tabular}

Tabelle A39: Der Heizstrahler ist wichtiger als das Baby zu trocknen und einzuhüllen $\mathrm{t} 1 \mathrm{vs.} \mathrm{t} 3$

2) Ein notfallmäßiger venöser Zugang ist am besten über die Nabelvene zu bekommen

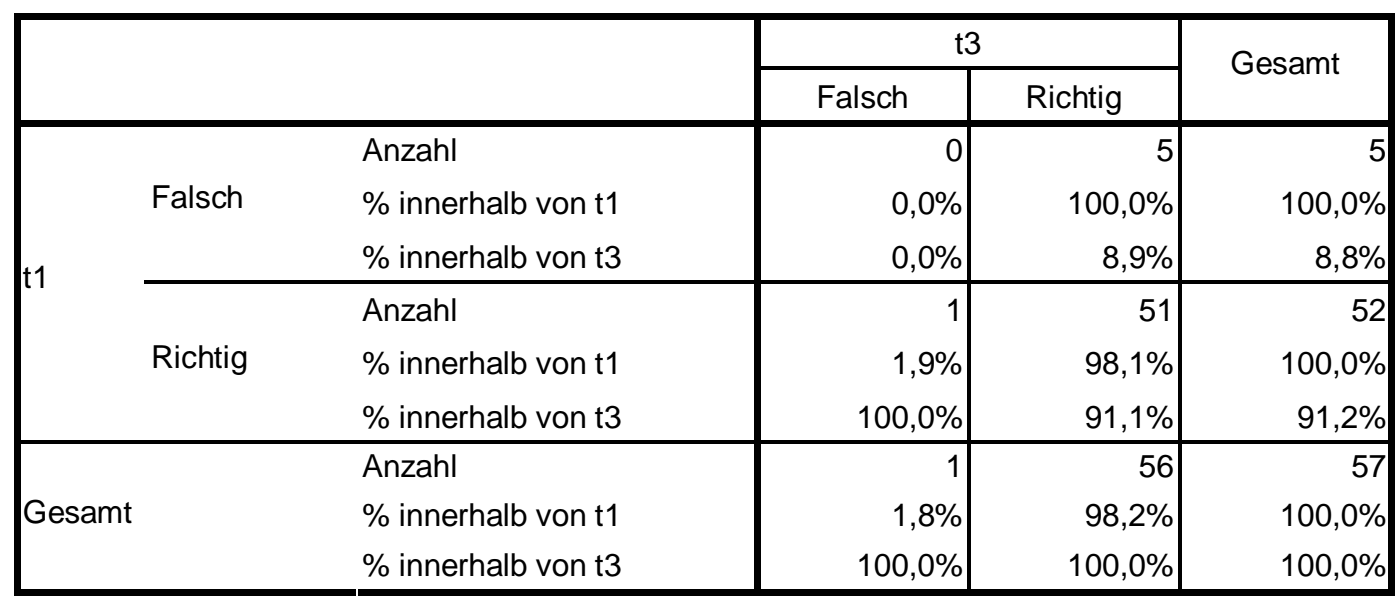

Tabelle A40: Ein notfallmäßiger venöser Zugang ist am besten über die Nabelvene zu bekommen t1 vs. t3

\begin{tabular}{|l|r|c|}
\hline & Wert & $\begin{array}{c}\text { Exakte Signifikanz } \\
\text { (2-seitig) }\end{array}$ \\
\hline $\begin{array}{l}\text { McNemar-Test } \\
\text { Anzahl der gültigen Fälle }\end{array}$ & 57 & $\mathbf{0 , 2 1 9}$ \\
\hline
\end{tabular}

Tabelle A41: McNemar-Test 
Der Haupteffekt von Adrenalin ist die Vasodilatation

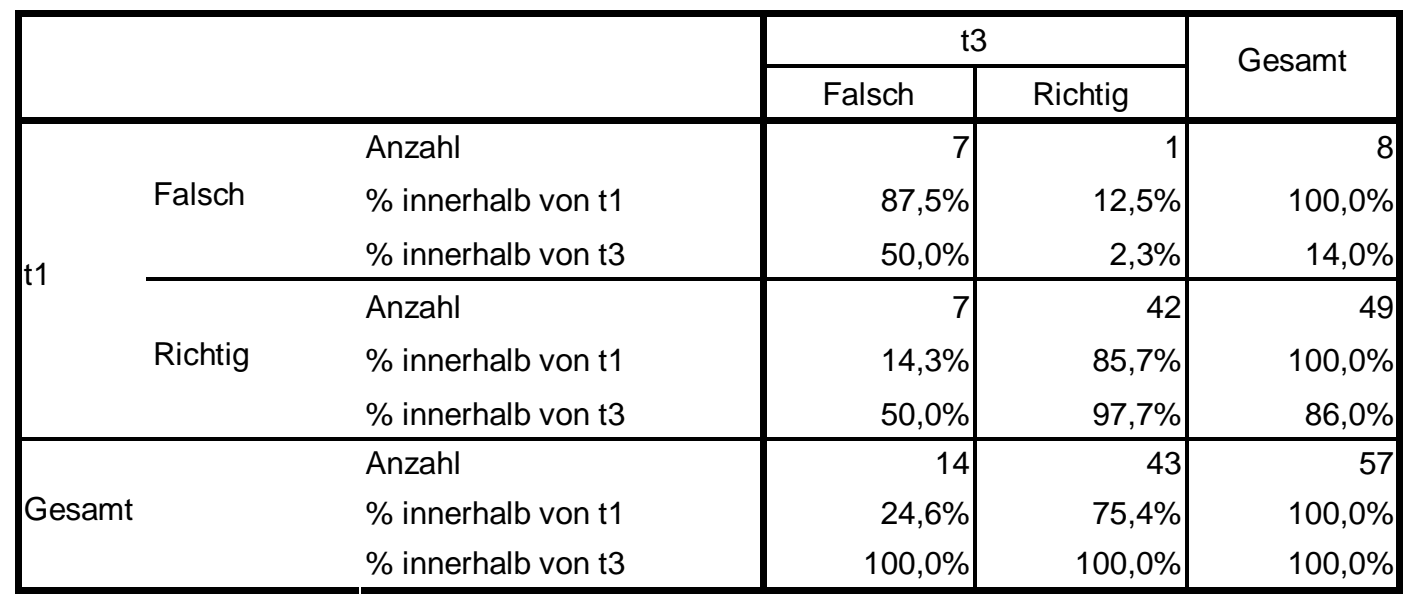

Tabelle A42: Der Haupteffekt von Adrenalin ist die Vasodilatation t1 vs. t3

\begin{tabular}{|l|r|c|}
\hline & Wert & $\begin{array}{c}\text { Exakte Signifikanz } \\
\text { (2-seitig) }\end{array}$ \\
\hline $\begin{array}{l}\text { McNemar-Test } \\
\text { Anzahl der gültigen Fälle }\end{array}$ & 57 & $\mathbf{0 , 0 7 0}$ \\
\hline
\end{tabular}

Tabelle A43: McNemar-Test

\section{4) Bronchiallavage verbessert immer die Prognose bei Mekoniumaspiration}

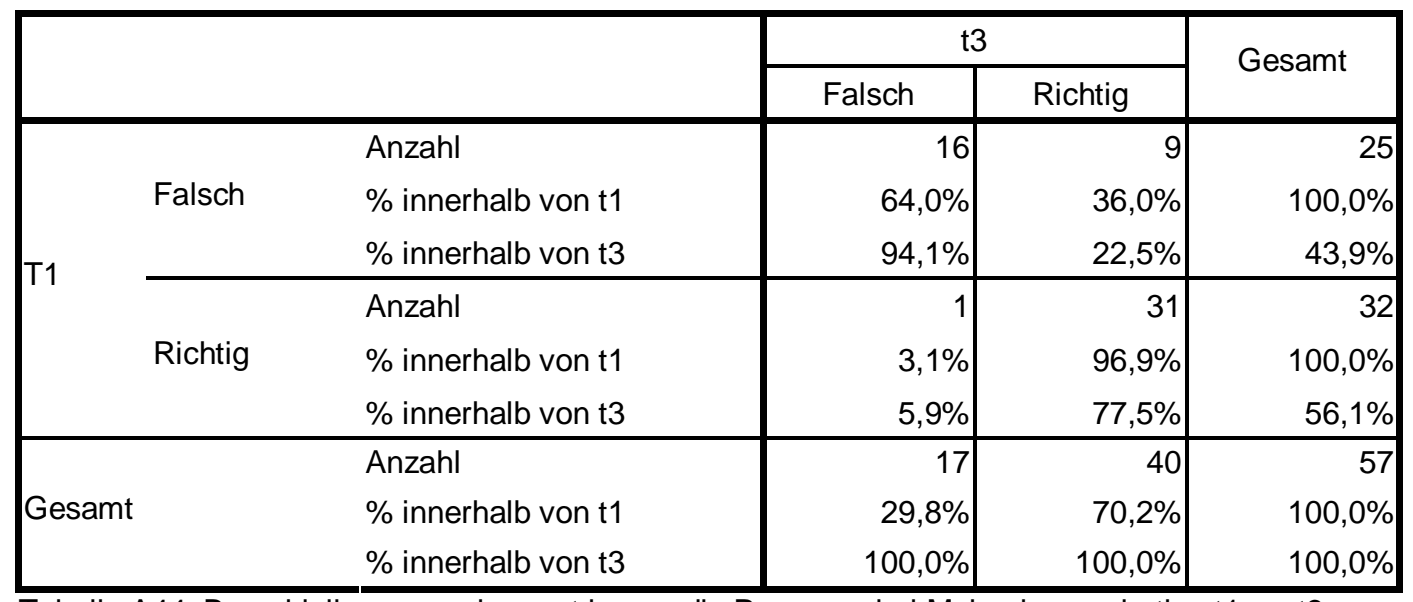

Tabelle A44: Bronchiallavage verbessert immer die Prognose bei Mekoniumaspiration t1 vs. t3

\begin{tabular}{|l|r|c|}
\hline & Wert & $\begin{array}{c}\text { Exakte Signifikanz } \\
\text { (2-seitig) }\end{array}$ \\
\hline $\begin{array}{l}\text { McNemar-Test } \\
\text { Anzahl der gültigen Fälle }\end{array}$ & 57 & $\mathbf{0 , 0 2 1}$ \\
\hline
\end{tabular}

Tabelle A45: McNemar-Test 
5) Herzmassage ist sinnvoll, bevor die Lunge sich aufgebläht hat

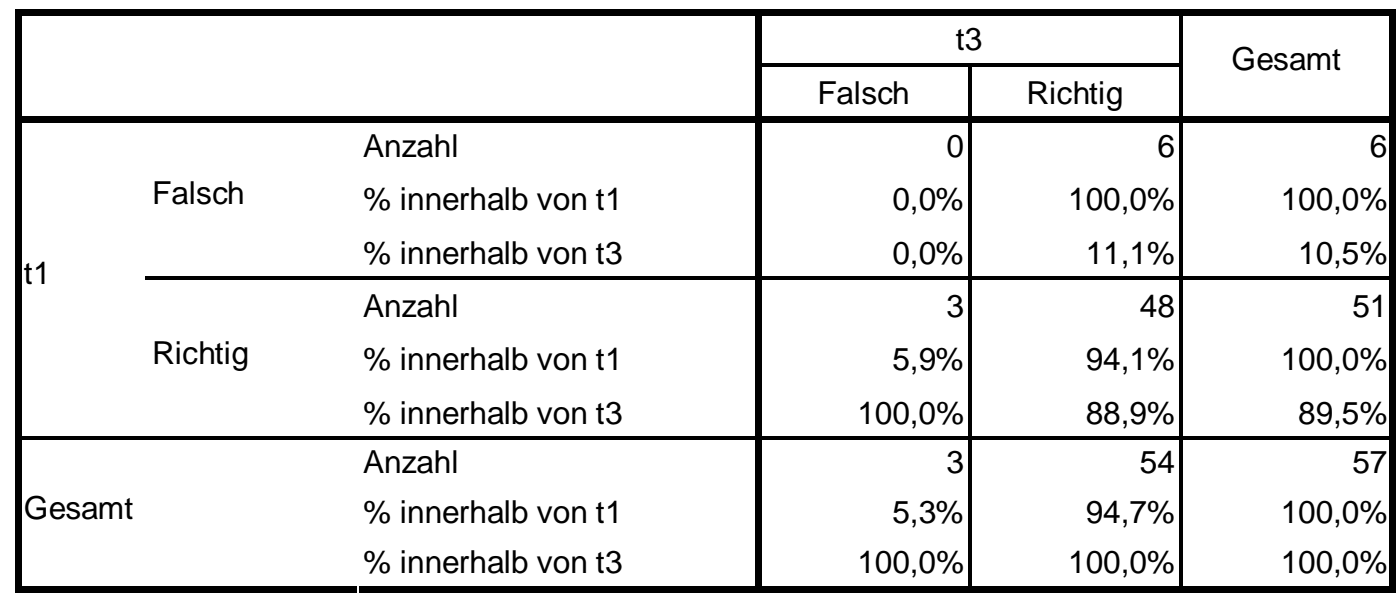

Tabelle A46: Herzmassage ist sinnvoll, bevor die Lunge sich aufgebläht hat t1 vs. t3

\begin{tabular}{|l|r|c|}
\hline & Wert & $\begin{array}{c}\text { Exakte Signifikanz } \\
\text { (2-seitig) }\end{array}$ \\
\hline $\begin{array}{l}\text { McNemar-Test } \\
\text { Anzahl der gültigen Fälle }\end{array}$ & 57 & $\mathbf{0 , 5 0 8}$ \\
\hline
\end{tabular}

Tabelle A47: McNemar-Test

6) Die Atmung stoppt üblicherweise, bevor der Kreislauf versagt

\begin{tabular}{|c|c|c|c|c|c|}
\hline & & & \multicolumn{2}{|c|}{ t3 } & \multirow{2}{*}{ Gesamt } \\
\hline & & & Falsch & Richtig & \\
\hline \multirow{6}{*}{ t1 } & \multirow{3}{*}{ Falsch } & Anzahl & 2 & 13 & 15 \\
\hline & & $\%$ innerhalb von $\mathrm{t} 1$ & $13,3 \%$ & $86,7 \%$ & $100,0 \%$ \\
\hline & & $\%$ innerhalb von $\mathrm{t} 3$ & $20,0 \%$ & $27,7 \%$ & $26,3 \%$ \\
\hline & \multirow{3}{*}{ Richtig } & Anzahl & 8 & 34 & 42 \\
\hline & & $\%$ innerhalb von $\mathrm{t} 1$ & $19,0 \%$ & $81,0 \%$ & $100,0 \%$ \\
\hline & & $\%$ innerhalb von $\mathrm{t} 3$ & $80,0 \%$ & $72,3 \%$ & $73,7 \%$ \\
\hline \multirow{3}{*}{\multicolumn{2}{|c|}{ Gesamt }} & Anzahl & 10 & 47 & 57 \\
\hline & & $\%$ innerhalb von $\mathrm{t} 1$ & $17,5 \%$ & $82,5 \%$ & $100,0 \%$ \\
\hline & & $\%$ innerhalb von $\mathrm{t} 3$ & $100,0 \%$ & $100,0 \%$ & $100,0 \%$ \\
\hline
\end{tabular}

Tabelle A48: Die Atmung stoppt üblicherweise, bevor der Kreislauf versagt t1 vs. t3

\begin{tabular}{|l|r|c|}
\hline & Wert & $\begin{array}{c}\text { Exakte Signifikanz } \\
\text { (2-seitig) }\end{array}$ \\
\hline $\begin{array}{l}\text { McNemar-Test } \\
\text { Anzahl der gültigen Fälle }\end{array}$ & 57 & $\mathbf{0 , 3 8 3}$ \\
\hline
\end{tabular}

Tabelle A49: McNemar-Test 
7) Ein am Termin Geborenes kann mehr als $100 \mathrm{ml}$ Fruchtwasser ohne Hilfe beseitigen

\begin{tabular}{|c|c|c|c|c|c|}
\hline & & & \multicolumn{2}{|c|}{ t3 } & \multirow{2}{*}{ Gesamt } \\
\hline & & & Falsch & Richtig & \\
\hline \multirow{6}{*}{$\mathrm{t} 1$} & \multirow{3}{*}{ Falsch } & Anzahl & 6 & 15 & 21 \\
\hline & & $\%$ innerhalb von $\mathrm{t} 1$ & $28,6 \%$ & $71,4 \%$ & $100,0 \%$ \\
\hline & & $\%$ innerhalb von $\mathrm{t} 3$ & $66,7 \%$ & $31,3 \%$ & $36,8 \%$ \\
\hline & \multirow{3}{*}{ Richtig } & Anzahl & 3 & 33 & 36 \\
\hline & & $\%$ innerhalb von $\mathrm{t} 1$ & $8,3 \%$ & $91,7 \%$ & $100,0 \%$ \\
\hline & & $\%$ innerhalb von $\mathrm{t} 3$ & $33,3 \%$ & $68,8 \%$ & $63,2 \%$ \\
\hline \multirow{3}{*}{\multicolumn{2}{|c|}{ Gesamt }} & Anzahl & 9 & 48 & 57 \\
\hline & & $\%$ innerhalb von $\mathrm{t} 1$ & $15,8 \%$ & $84,2 \%$ & $100,0 \%$ \\
\hline & & $\%$ innerhalb von $\mathrm{t} 3$ & $100,0 \%$ & $100,0 \%$ & $100,0 \%$ \\
\hline
\end{tabular}

Tabelle A50: Ein am Termin Geborenes kann mehr als 100 ml Fruchtwasser ohne Hilfe beseitigen t1 vs. t3

\begin{tabular}{|l|r|c|}
\hline & Wert & $\begin{array}{c}\text { Exakte Signifikanz } \\
\text { (2-seitig) }\end{array}$ \\
\hline $\begin{array}{l}\text { McNemar-Test } \\
\text { Anzahl der gültigen Fälle }\end{array}$ & 57 & $\mathbf{0 , 0 0 8}$ \\
\hline
\end{tabular}

Tabelle A51: McNemar-Test

8) Ein Säugling mit Herzfrequenz unter 60/min muss sofort intubiert werden

\begin{tabular}{|c|c|c|c|c|c|}
\hline & & & \multicolumn{2}{|c|}{ t3 } & \multirow{2}{*}{ Gesamt } \\
\hline & & & Falsch & Richtig & \\
\hline \multirow{6}{*}{$\mathrm{t} 1$} & \multirow{3}{*}{ Falsch } & Anzahl & 3 & 6 & 9 \\
\hline & & $\%$ innerhalb von $\mathrm{t} 1$ & $33,3 \%$ & $66,7 \%$ & $100,0 \%$ \\
\hline & & $\%$ innerhalb von $\mathrm{t} 3$ & $75,0 \%$ & $11,3 \%$ & $15,8 \%$ \\
\hline & \multirow{3}{*}{ Richtig } & Anzahl & 1 & 47 & 48 \\
\hline & & $\%$ innerhalb von $\mathrm{t} 1$ & $2,1 \%$ & $97,9 \%$ & $100,0 \%$ \\
\hline & & $\%$ innerhalb von $\mathrm{t} 3$ & $25,0 \%$ & $88,7 \%$ & $84,2 \%$ \\
\hline \multirow{3}{*}{\multicolumn{2}{|c|}{ Gesamt }} & Anzahl & 4 & 53 & 57 \\
\hline & & $\%$ innerhalb von $\mathrm{t} 1$ & $7,0 \%$ & $93,0 \%$ & $100,0 \%$ \\
\hline & & $\%$ innerhalb von $\mathrm{t} 3$ & $100,0 \%$ & $100,0 \%$ & $100,0 \%$ \\
\hline
\end{tabular}

Tabelle A52: Ein Säugling mit Herzfrequenz unter 60/min muss sofort intubiert werden t1 vs. t3

\begin{tabular}{|l|r|c|}
\hline & Wert & $\begin{array}{c}\text { Exakte Signifikanz } \\
\text { (2-seitig) }\end{array}$ \\
\hline $\begin{array}{l}\text { McNemar-Test } \\
\text { Anzahl der gültigen Fälle }\end{array}$ & 57 & $\mathbf{0 , 1 2 5}$ \\
\hline
\end{tabular}

Tabelle A53: McNemar-Test 
9) Thoraxkompressionen sollten den Thorax 1/3 des Weges bis zum Rücken eindrücken

\begin{tabular}{|c|c|c|c|c|c|}
\hline & & & \multicolumn{2}{|c|}{ t3 } & \multirow{2}{*}{ Gesamt } \\
\hline & & & Falsch & Richtig & \\
\hline \multirow{6}{*}{$\mathrm{t} 1$} & \multirow{3}{*}{ Falsch } & Anzahl & 0 & 6 & 6 \\
\hline & & $\%$ innerhalb von $\mathrm{t} 1$ & $0,0 \%$ & $100,0 \%$ & $100,0 \%$ \\
\hline & & $\%$ innerhalb von t3 & $0,0 \%$ & $10,9 \%$ & $10,5 \%$ \\
\hline & \multirow{3}{*}{ Richtig } & Anzahl & 2 & 49 & 51 \\
\hline & & $\%$ innerhalb von $\mathrm{t} 1$ & $3,9 \%$ & $96,1 \%$ & $100,0 \%$ \\
\hline & & $\%$ innerhalb von t3 & $100,0 \%$ & $89,1 \%$ & $89,5 \%$ \\
\hline \multirow{3}{*}{\multicolumn{2}{|c|}{ Gesamt }} & Anzahl & 2 & 55 & 57 \\
\hline & & $\%$ innerhalb von $\mathrm{t} 1$ & $3,5 \%$ & $96,5 \%$ & $100,0 \%$ \\
\hline & & $\%$ innerhalb von t3 & $100,0 \%$ & $100,0 \%$ & $100,0 \%$ \\
\hline
\end{tabular}

Tabelle A54: Thoraxkompressionen sollten den Thorax 1/3 des Weges bis zum Rücken eindrücken t1 vs. t3

\begin{tabular}{|l|r|c|}
\hline & Wert & $\begin{array}{c}\text { Exakte Signifikanz } \\
\text { (2-seitig) }\end{array}$ \\
\hline $\begin{array}{l}\text { McNemar-Test } \\
\text { Anzahl der gültigen Fälle }\end{array}$ & 57 & $\mathbf{0 , 2 8 9}$ \\
\hline
\end{tabular}

Tabelle A55: McNemar-Test

10) Systeme, die zur Beatmung von Neugeborenen benutzt werden, sollten ein Überdruckventil von $30-40 \mathrm{~cm}$ Wassersäule besitzen

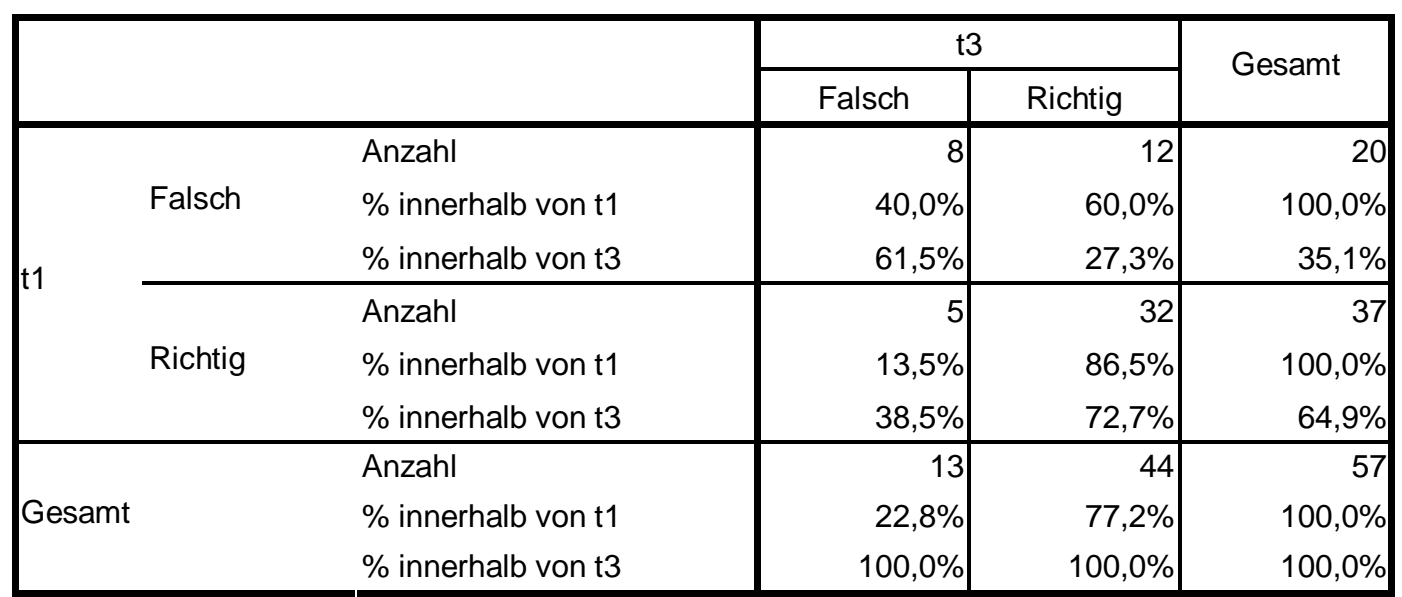

Tabelle A56: Systeme, die zur Beatmung von Neugeborenen benutzt werden, sollten ein Überdruckventil von 30$40 \mathrm{~cm}$ Wassersäule besitzen $\mathrm{t} 1$ vs. $\mathrm{t} 3$

\begin{tabular}{|l|r|c|}
\hline & Wert & $\begin{array}{c}\text { Exakte Signifikanz } \\
\text { (2-seitig) }\end{array}$ \\
\hline $\begin{array}{l}\text { McNemar-Test } \\
\text { Anzahl der gültigen Fälle }\end{array}$ & 57 & $\mathbf{0 , 1 4 3}$ \\
\hline
\end{tabular}

Tabelle A57: McNemar-Test 
Alle Kinder, die bei Geburt intubiert werden, müssen auf eine Neugeborenenintensivstation übernommen werden

\begin{tabular}{|c|c|c|c|c|c|}
\hline & & & \multicolumn{2}{|c|}{ t3 } & \multirow{2}{*}{ Gesamt } \\
\hline & & & Falsch & Richtig & \\
\hline \multirow{6}{*}{ t1 } & \multirow{3}{*}{ Falsch } & Anzahl & 0 & 2 & 2 \\
\hline & & $\%$ innerhalb von $t 1$ & $0,0 \%$ & $100,0 \%$ & $100,0 \%$ \\
\hline & & $\%$ innerhalb von t3 & $0,0 \%$ & $3,8 \%$ & $3,5 \%$ \\
\hline & \multirow{3}{*}{ Richtig } & Anzahl & 5 & 50 & 55 \\
\hline & & $\%$ innerhalb von $\mathrm{t} 1$ & $9,1 \%$ & $90,9 \%$ & $100,0 \%$ \\
\hline & & $\%$ innerhalb von $\mathrm{t} 3$ & $100,0 \%$ & $96,2 \%$ & $96,5 \%$ \\
\hline \multirow{3}{*}{\multicolumn{2}{|c|}{ Gesamt }} & Anzahl & 5 & 52 & 57 \\
\hline & & $\%$ innerhalb von $\mathrm{t} 1$ & $8,8 \%$ & $91,2 \%$ & $100,0 \%$ \\
\hline & & $\%$ innerhalb von $\mathrm{t} 3$ & $100,0 \%$ & $100,0 \%$ & $100,0 \%$ \\
\hline
\end{tabular}

Tabelle A58: Alle Kinder, die bei Geburt intubiert werden, müssen auf eine Neugeborenenintensivstation übernommen werden t1 vs. $\mathrm{t} 3$

\begin{tabular}{|l|r|c|}
\hline & Wert & $\begin{array}{c}\text { Exakte Signifikanz } \\
\text { (2-seitig) }\end{array}$ \\
\hline $\begin{array}{l}\text { McNemar-Test } \\
\text { Anzahl der gültigen Fälle }\end{array}$ & 57 & $\mathbf{0 , 4 5 3}$ \\
\hline
\end{tabular}

Tabelle A59: McNemar-Test

12) Hypothermie kann Apnoen verursachen

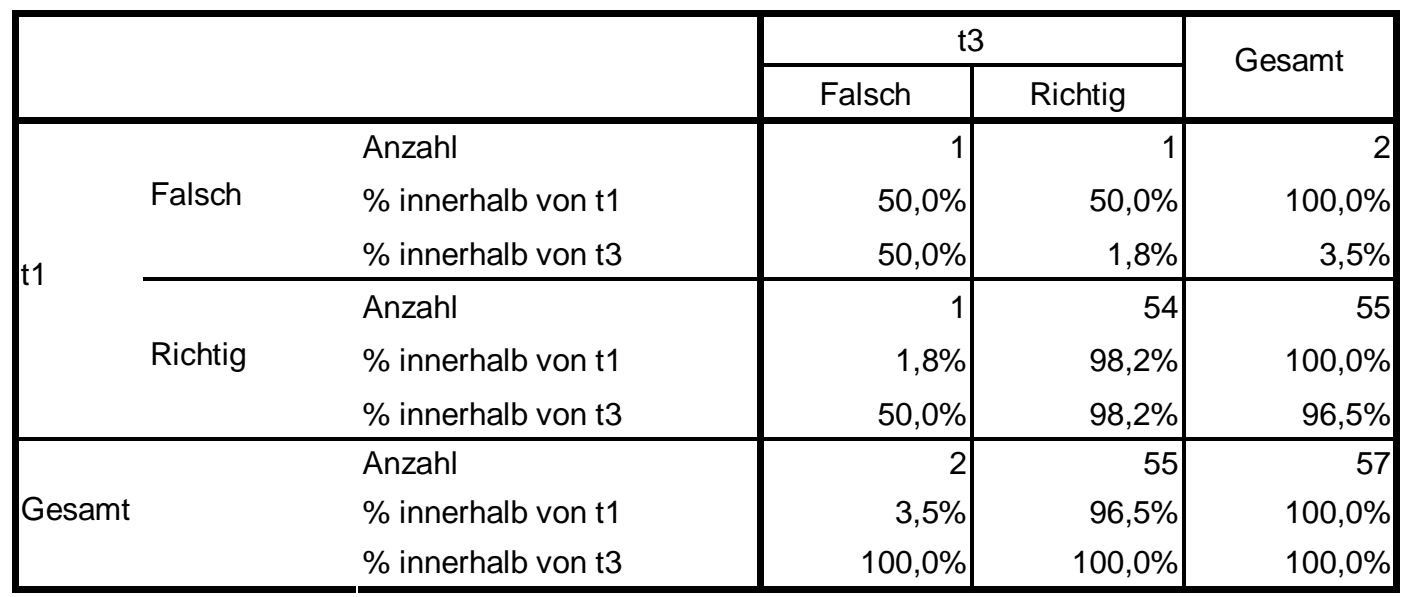

Tabelle A60: Hypothermie kann Apnoen verursachen t1 vs. t3

\begin{tabular}{|l|r|c|}
\hline & Wert & $\begin{array}{c}\text { Exakte Signifikanz } \\
\text { (2-seitig) }\end{array}$ \\
\hline $\begin{array}{l}\text { McNemar-Test } \\
\text { Anzahl der gültigen Fälle }\end{array}$ & 57 & $\mathbf{1 , 0 0 0}$ \\
\hline
\end{tabular}

Tabelle A61: McNemar-Test 
13) Es ist ratsam, die Reanimation bei einem Frühgeborenen mit einem niedrigeren Beatmungsdruck zu starten als bei einem reifen Neugeborenen

\begin{tabular}{|c|c|c|c|c|c|}
\hline & & & \multicolumn{2}{|c|}{ t3 } & \multirow{2}{*}{ Gesamt } \\
\hline & & & Falsch & Richtig & \\
\hline \multirow{6}{*}{$\mathrm{t} 1$} & \multirow{3}{*}{ Falsch } & Anzahl & 5 & 8 & 13 \\
\hline & & $\%$ innerhalb von $\mathrm{t} 1$ & $38,5 \%$ & $61,5 \%$ & $100,0 \%$ \\
\hline & & $\%$ innerhalb von t3 & $71,4 \%$ & $16,0 \%$ & $22,8 \%$ \\
\hline & \multirow{3}{*}{ Richtig } & Anzahl & 2 & 42 & 44 \\
\hline & & $\%$ innerhalb von $\mathrm{t} 1$ & $4,5 \%$ & $95,5 \%$ & $100,0 \%$ \\
\hline & & $\%$ innerhalb von t3 & $28,6 \%$ & $84,0 \%$ & $77,2 \%$ \\
\hline \multirow{3}{*}{\multicolumn{2}{|c|}{ Gesamt }} & Anzahl & 7 & 50 & 57 \\
\hline & & $\%$ innerhalb von $\mathrm{t} 1$ & $12,3 \%$ & $87,7 \%$ & $100,0 \%$ \\
\hline & & $\%$ innerhalb von $\mathrm{t} 3$ & $100,0 \%$ & $100,0 \%$ & $100,0 \%$ \\
\hline
\end{tabular}

Tabelle A62: Es ist ratsam, die Reanimation bei einem Frühgeborenen mit einem niedrigeren Beatmungsdruck zu starten als bei einem reifen Neugeborenen t1 vs. $\mathrm{t} 3$

\begin{tabular}{|l|r|c|}
\hline & Wert & $\begin{array}{c}\text { Exakte Signifikanz } \\
\text { (2-seitig) }\end{array}$ \\
\hline $\begin{array}{l}\text { McNemar-Test } \\
\text { Anzahl der gültigen Fälle }\end{array}$ & 57 & $\mathbf{0 , 1 0 9}$ \\
\hline
\end{tabular}

Tabelle A63: McNemar-Test

14) Maskenbeatmung ist viel weniger effektiv als Beatmung nach Intubation

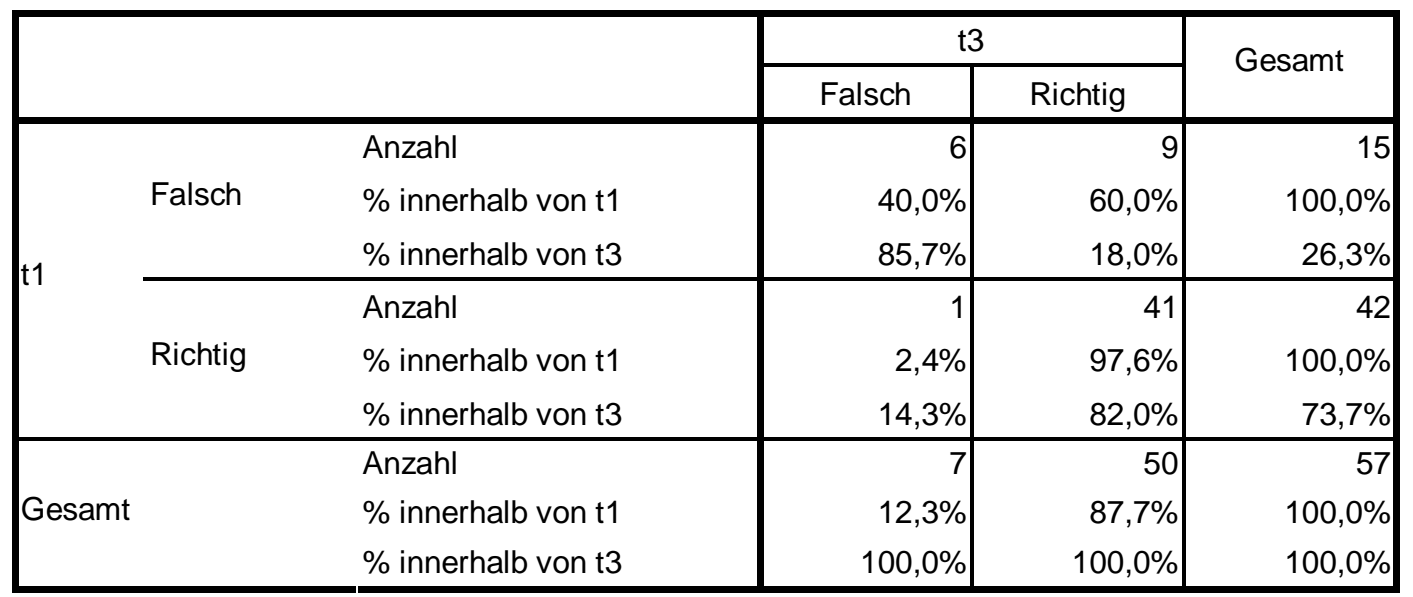

Tabelle A64: Maskenbeatmung ist viel weniger effektiv als Beatmung nach Intubation t1 vs. t3

\begin{tabular}{|l|r|c|}
\hline & Wert & $\begin{array}{c}\text { Exakte Signifikanz } \\
\text { (2-seitig) }\end{array}$ \\
\hline $\begin{array}{l}\text { McNemar-Test } \\
\text { Anzahl der gültigen Fälle }\end{array}$ & 57 & $\mathbf{0 , 0 2 1}$ \\
\hline
\end{tabular}

Tabelle A65: McNemar-Test 
15) Die initiale Reanimation eines Frühgeborenen unterscheidet sich sehr von einer Reanimation bei einem Reifgeborenen

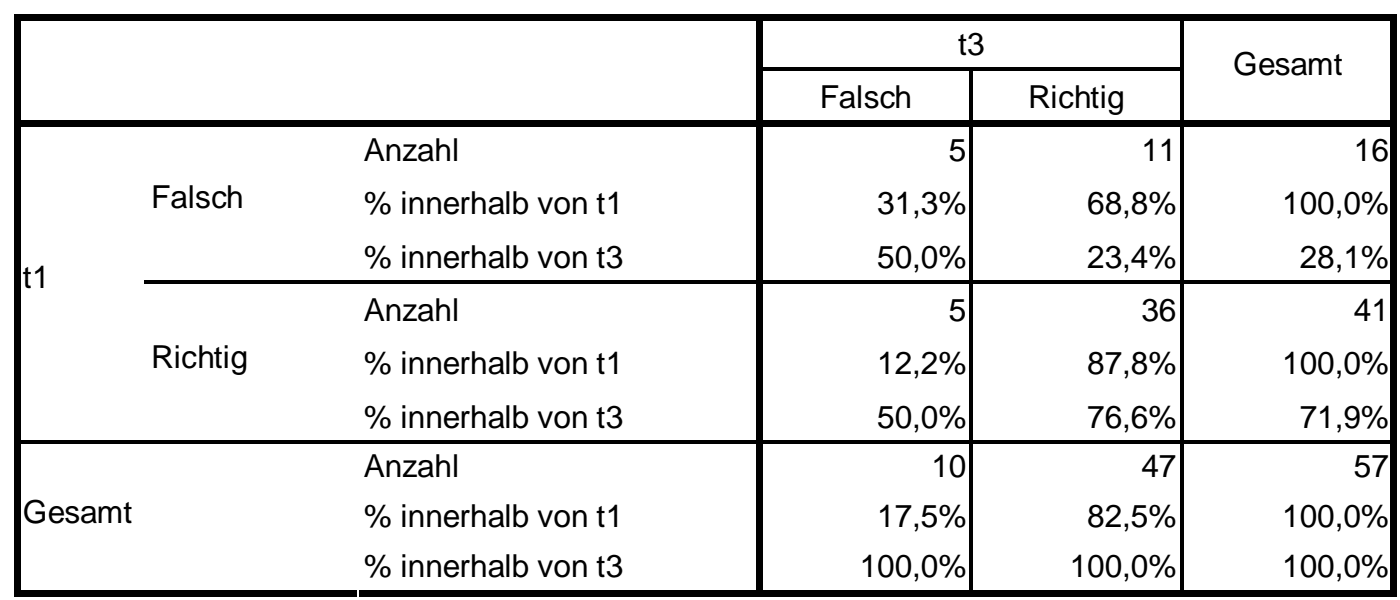

Tabelle A66: Die initiale Reanimation eines Frühgeborenen unterscheidet sich sehr von einer Reanimation bei einem Reifgeborenen t1 vs. $\mathrm{t} 3$

\begin{tabular}{|l|r|c|}
\hline & Wert & $\begin{array}{c}\text { Exakte Signifikanz } \\
\text { (2-seitig) }\end{array}$ \\
\hline $\begin{array}{l}\text { McNemar-Test } \\
\text { Anzahl der gültigen Fälle }\end{array}$ & 57 & $\mathbf{0 , 2 1 0}$ \\
\hline
\end{tabular}

Tabelle A67: McNemar-Test

16) Schnappatmung ist immer flach

\begin{tabular}{|c|c|c|c|c|c|}
\hline & & & \multicolumn{2}{|c|}{ t3 } & \multirow{2}{*}{ Gesamt } \\
\hline & & & Falsch & Richtig & \\
\hline \multirow{6}{*}{$\mathrm{t} 1$} & \multirow{3}{*}{ Falsch } & Anzahl & 6 & 5 & 11 \\
\hline & & $\%$ innerhalb von $\mathrm{t} 1$ & $54,5 \%$ & $45,5 \%$ & $100,0 \%$ \\
\hline & & $\%$ innerhalb von $\mathrm{t} 3$ & $37,5 \%$ & $12,2 \%$ & $19,3 \%$ \\
\hline & \multirow{3}{*}{ Richtig } & Anzahl & 10 & 36 & 46 \\
\hline & & $\%$ innerhalb von $\mathrm{t} 1$ & $21,7 \%$ & $78,3 \%$ & $100,0 \%$ \\
\hline & & $\%$ innerhalb von $\mathrm{t} 3$ & $62,5 \%$ & $87,8 \%$ & $80,7 \%$ \\
\hline \multirow{3}{*}{\multicolumn{2}{|c|}{ Gesamt }} & Anzahl & 16 & 41 & 57 \\
\hline & & $\%$ innerhalb von $\mathrm{t} 1$ & $28,1 \%$ & $71,9 \%$ & $100,0 \%$ \\
\hline & & $\%$ innerhalb von $\mathrm{t} 3$ & $100,0 \%$ & $100,0 \%$ & $100,0 \%$ \\
\hline
\end{tabular}

Tabelle A68: Schnappatmung ist immer flach t1 vs. t3

\begin{tabular}{|l|r|c|}
\hline & Wert & $\begin{array}{c}\text { Exakte Signifikanz } \\
\text { (2-seitig) }\end{array}$ \\
\hline $\begin{array}{l}\text { McNemar-Test } \\
\text { Anzahl der gültigen Fälle }\end{array}$ & 57 & $\mathbf{0 , 3 0 2}$ \\
\hline
\end{tabular}

Tabelle A69: McNemar-Test 
17) Nach einer Reanimation ist wichtig: Abnahme von Nabelschnurblut und venösem Blut zur Messung von pH und BE

\begin{tabular}{|c|c|c|c|c|c|}
\hline & & & \multicolumn{2}{|c|}{ t3 } & \multirow{2}{*}{ Gesamt } \\
\hline & & & Falsch & Richtig & \\
\hline \multirow{6}{*}{$t 1$} & \multirow{3}{*}{ Falsch } & Anzahl & 0 & 4 & 4 \\
\hline & & $\%$ innerhalb von $\mathrm{t} 1$ & $0,0 \%$ & $100,0 \%$ & $100,0 \%$ \\
\hline & & $\%$ innerhalb von t3 & $0,0 \%$ & $8,0 \%$ & $7,0 \%$ \\
\hline & \multirow{3}{*}{ Richtig } & Anzahl & 7 & 46 & 53 \\
\hline & & $\%$ innerhalb von $\mathrm{t} 1$ & $13,2 \%$ & $86,8 \%$ & $100,0 \%$ \\
\hline & & $\%$ innerhalb von t3 & $100,0 \%$ & $92,0 \%$ & $93,0 \%$ \\
\hline \multirow{3}{*}{\multicolumn{2}{|c|}{ Gesamt }} & Anzahl & 7 & 50 & 57 \\
\hline & & $\%$ innerhalb von $\mathrm{t} 1$ & $12,3 \%$ & $87,7 \%$ & $100,0 \%$ \\
\hline & & $\%$ innerhalb von t3 & $100,0 \%$ & $100,0 \%$ & $100,0 \%$ \\
\hline
\end{tabular}

Tabelle A70: Nach einer Reanimation ist wichtig: Abnahme von Nabelschnurblut und venösem Blut zur Messung von $\mathrm{pH}$ und $\mathrm{BE} \mathrm{t} 1$ vs. t3

\begin{tabular}{|l|r|c|}
\hline & Wert & $\begin{array}{c}\text { Exakte Signifikanz } \\
\text { (2-seitig) }\end{array}$ \\
\hline $\begin{array}{l}\text { McNemar-Test } \\
\text { Anzahl der gültigen Fälle }\end{array}$ & 57 & $\mathbf{0 , 5 4 9}$ \\
\hline
\end{tabular}

Tabelle A71: McNemar-Test

\section{c) Vergleich direkt nach Schulung (t2) vs. nach 6 Monaten (t3) (57 Fälle)}

1) Der Heizstrahler ist wichtiger als das Baby zu trocknen und einzuhüllen

\begin{tabular}{|c|c|c|c|c|}
\hline & & & t3 & \multirow{2}{*}{ Gesamt } \\
\hline & & & Richtig & \\
\hline \multirow{6}{*}{$t 2$} & \multirow{3}{*}{ Falsch } & Anzahl & 1 & 1 \\
\hline & & $\%$ innerhalb von t2 & $100,0 \%$ & $100,0 \%$ \\
\hline & & $\%$ innerhalb von t3 & $1,8 \%$ & $1,8 \%$ \\
\hline & \multirow{3}{*}{ Richtig } & Anzahl & 56 & 56 \\
\hline & & $\%$ innerhalb von t2 & $100,0 \%$ & $100,0 \%$ \\
\hline & & $\%$ innerhalb von $\mathrm{t} 3$ & $98,2 \%$ & $98,2 \%$ \\
\hline \multirow{3}{*}{\multicolumn{2}{|c|}{ Gesamt }} & Anzahl & 57 & 57 \\
\hline & & $\%$ innerhalb von t2 & $100,0 \%$ & $100,0 \%$ \\
\hline & & $\%$ innerhalb von $\mathrm{t} 3$ & $100,0 \%$ & $100,0 \%$ \\
\hline
\end{tabular}

Tabelle A72: Der Heizstrahler ist wichtiger als das Baby zu trocknen und einzuhüllen t2 vs. t3 
2) Ein notfallmäßiger venöser Zugang ist am besten über die Nabelvene zu bekommen

\begin{tabular}{|c|c|c|c|c|c|}
\hline & & & \multicolumn{2}{|c|}{ t3 } & \multirow{2}{*}{ Gesamt } \\
\hline & & & Falsch & Richtig & \\
\hline \multirow{6}{*}{ t2 } & \multirow{3}{*}{ Falsch } & Anzahl & 0 & 1 & 1 \\
\hline & & $\%$ innerhalb von $\mathrm{t} 2$ & $0,0 \%$ & $100,0 \%$ & $100,0 \%$ \\
\hline & & $\%$ innerhalb von t3 & $0,0 \%$ & $1,8 \%$ & $1,8 \%$ \\
\hline & \multirow{3}{*}{ Richtig } & Anzahl & 1 & 55 & 56 \\
\hline & & $\%$ innerhalb von $\mathrm{t} 2$ & $1,8 \%$ & $98,2 \%$ & $100,0 \%$ \\
\hline & & $\%$ innerhalb von $\mathrm{t} 3$ & $100,0 \%$ & $98,2 \%$ & $98,2 \%$ \\
\hline \multirow{3}{*}{\multicolumn{2}{|c|}{ Gesamt }} & Anzahl & 1 & 56 & 57 \\
\hline & & $\%$ innerhalb von $\mathrm{t} 2$ & $1,8 \%$ & $98,2 \%$ & $100,0 \%$ \\
\hline & & $\%$ innerhalb von $\mathrm{t} 3$ & $100,0 \%$ & $100,0 \%$ & $100,0 \%$ \\
\hline
\end{tabular}

Tabelle A73: Ein notfallmäßiger venöser Zugang ist am besten über die Nabelvene zu bekommen t2 vs. t3

\begin{tabular}{|l|r|c|}
\hline & Wert & $\begin{array}{c}\text { Exakte Signifikanz } \\
\text { (2-seitig) }\end{array}$ \\
\hline $\begin{array}{l}\text { McNemar-Test } \\
\text { Anzahl der gültigen Fälle }\end{array}$ & 57 & $\mathbf{1 , 0 0 0}$ \\
\hline
\end{tabular}

Tabelle A74: McNemar-Test

\section{3) Der Haupteffekt von Adrenalin ist die Vasodilatation}

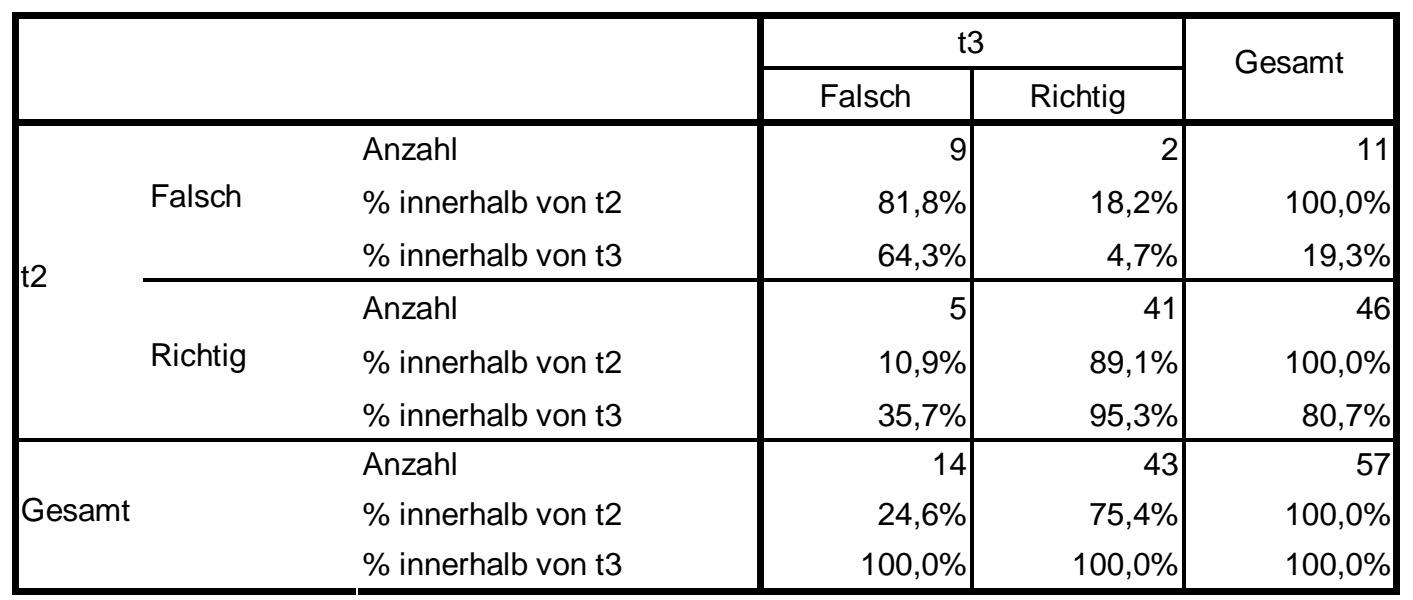

Tabelle A75: Der Haupteffekt von Adrenalin ist die Vasodilatation t2 vs. t3

\begin{tabular}{|l|r|c|}
\hline & Wert & $\begin{array}{c}\text { Exakte Signifikanz } \\
\text { (2-seitig) }\end{array}$ \\
\hline $\begin{array}{l}\text { McNemar-Test } \\
\text { Anzahl der gültigen Fälle }\end{array}$ & 57 & $\mathbf{0 , 4 5 3}$ \\
\hline
\end{tabular}

Tabelle A76: McNemar-Test 
4) Bronchiallavage verbessert immer die Prognose bei Mekoniumaspiration

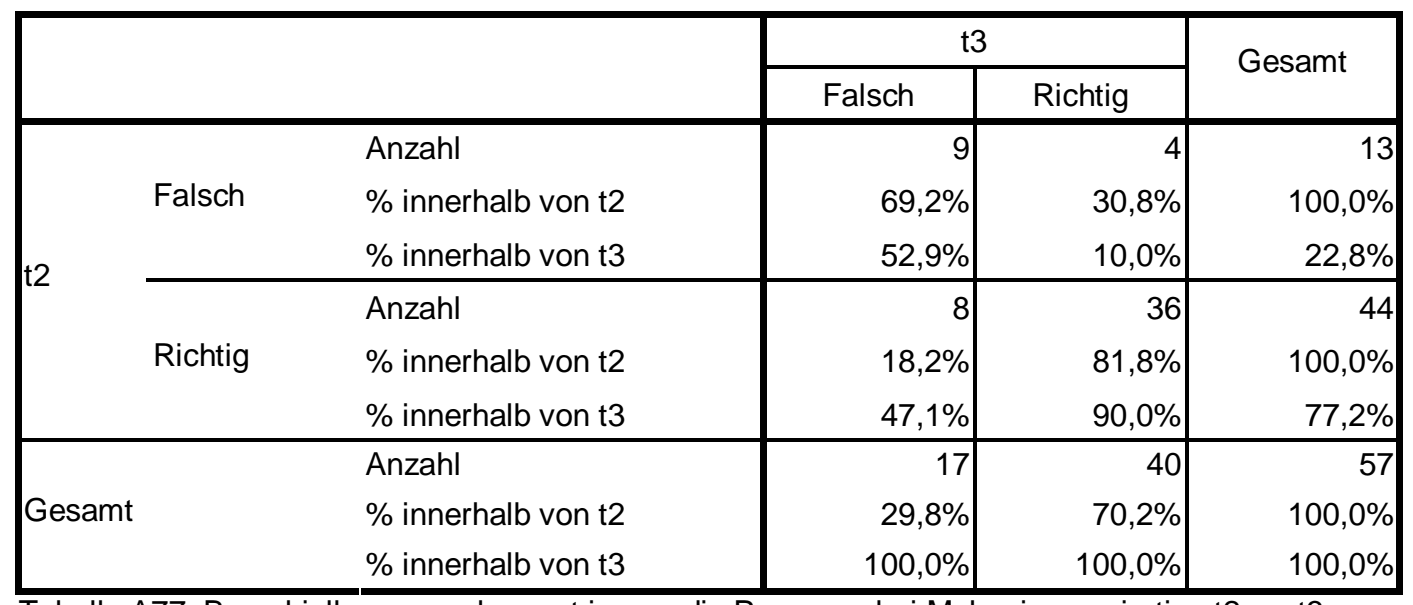

Tabelle A77: Bronchiallavage verbessert immer die Prognose bei Mekoniumaspiration t2 vs. t3

\begin{tabular}{|l|r|c|}
\hline & Wert & $\begin{array}{c}\text { Exakte Signifikanz } \\
\text { (2-seitig) }\end{array}$ \\
\hline $\begin{array}{l}\text { McNemar-Test } \\
\text { Anzahl der gültigen Fälle }\end{array}$ & 57 & $\mathbf{0 , 3 8 8}$ \\
\hline
\end{tabular}

Tabelle A78: McNemar-Test

5) Herzmassage ist sinnvoll, bevor die Lunge sich aufgebläht hat

\begin{tabular}{|c|c|c|c|c|c|}
\hline & & & \multicolumn{2}{|c|}{ t3 } & \multirow{2}{*}{ Gesamt } \\
\hline & & & Falsch & Richtig & \\
\hline \multirow{3}{*}{ t2 } & \multirow{3}{*}{ Richtig } & Anzahl & 3 & 54 & 57 \\
\hline & & $\%$ innerhalb von t2 & $5,3 \%$ & $94,7 \%$ & $100,0 \%$ \\
\hline & & $\%$ innerhalb von $\mathrm{t} 3$ & $100,0 \%$ & $100,0 \%$ & $100,0 \%$ \\
\hline \multirow{3}{*}{\multicolumn{2}{|c|}{ Gesamt }} & Anzahl & 3 & 54 & 57 \\
\hline & & $\%$ innerhalb von $t 2$ & $5,3 \%$ & $94,7 \%$ & $100,0 \%$ \\
\hline & & $\%$ innerhalb von $\mathrm{t} 3$ & $100,0 \%$ & $100,0 \%$ & $100,0 \%$ \\
\hline
\end{tabular}

Tabelle A79: Herzmassage ist sinnvoll, bevor die Lunge sich aufgebläht hat t2 vs. t3

6) Die Atmung stoppt üblicherweise, bevor der Kreislauf versagt

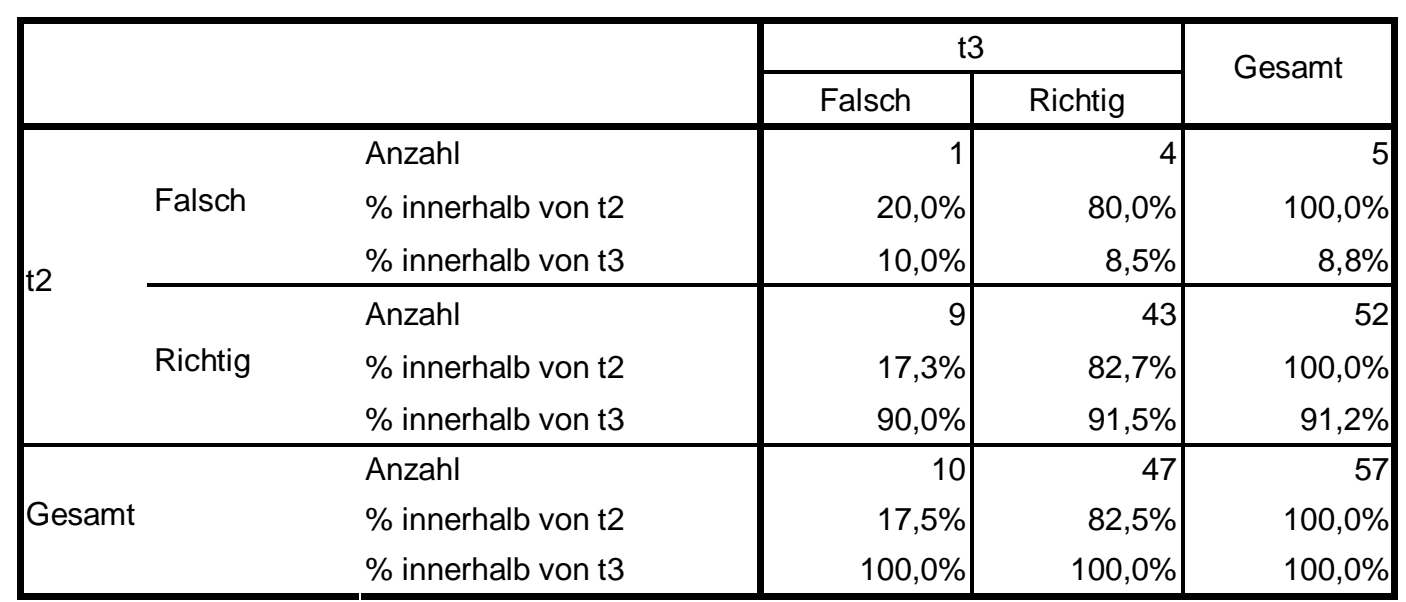

Tabelle A80: Die Atmung stoppt üblicherweise, bevor der Kreislauf versagt t2 vs. t3 


\begin{tabular}{|l|r|c|}
\hline & Wert & $\begin{array}{c}\text { Exakte Signifikanz } \\
\text { (2-seitig) }\end{array}$ \\
\hline $\begin{array}{l}\text { McNemar-Test } \\
\text { Anzahl der gültigen Fälle }\end{array}$ & 57 & $\mathbf{0 , 2 6 7}$ \\
\hline
\end{tabular}

Tabelle A81: McNemar-Test

7) Ein am Termin Geborenes kann mehr als $100 \mathrm{ml}$ Fruchtwasser ohne Hilfe beseitigen

\begin{tabular}{|c|c|c|c|c|c|}
\hline & & & \multicolumn{2}{|c|}{$\mathrm{t} 3$} & \multirow{2}{*}{ Gesamt } \\
\hline & & & Falsch & Richtig & \\
\hline \multirow{6}{*}{ t2 } & \multirow{3}{*}{ Falsch } & Anzahl & 5 & 3 & 8 \\
\hline & & $\%$ innerhalb von t2 & $62,5 \%$ & $37,5 \%$ & $100,0 \%$ \\
\hline & & $\%$ innerhalb von $\mathrm{t} 3$ & $55,6 \%$ & $6,3 \%$ & $14,0 \%$ \\
\hline & \multirow{3}{*}{ Richtig } & Anzahl & 4 & 45 & 49 \\
\hline & & $\%$ innerhalb von t2 & $8,2 \%$ & $91,8 \%$ & $100,0 \%$ \\
\hline & & $\%$ innerhalb von t3 & $44,4 \%$ & $93,8 \%$ & $86,0 \%$ \\
\hline \multirow{3}{*}{\multicolumn{2}{|c|}{ Gesamt }} & Anzahl & 9 & 48 & 57 \\
\hline & & $\%$ innerhalb von $\mathrm{t} 2$ & $15,8 \%$ & $84,2 \%$ & $100,0 \%$ \\
\hline & & $\%$ innerhalb von $\mathrm{t} 3$ & $100,0 \%$ & $100,0 \%$ & $100,0 \%$ \\
\hline
\end{tabular}

Tabelle A82: Ein am Termin Geborenes kann mehr als $100 \mathrm{ml}$ Fruchtwasser ohne Hilfe beseitigen t2 vs. t3

\begin{tabular}{|l|r|r|}
\hline & Wert & $\begin{array}{c}\text { Exakte Signifikanz } \\
\text { (2-seitig) }\end{array}$ \\
\hline $\begin{array}{l}\text { McNemar-Test } \\
\text { Anzahl der gültigen Fälle }\end{array}$ & 57 & $\mathbf{1 , 0 0 0}$ \\
\hline
\end{tabular}

Tabelle A83: McNemar-Test

8) Ein Säugling mit Herzfrequenz unter 60/min muss sofort intubiert werden

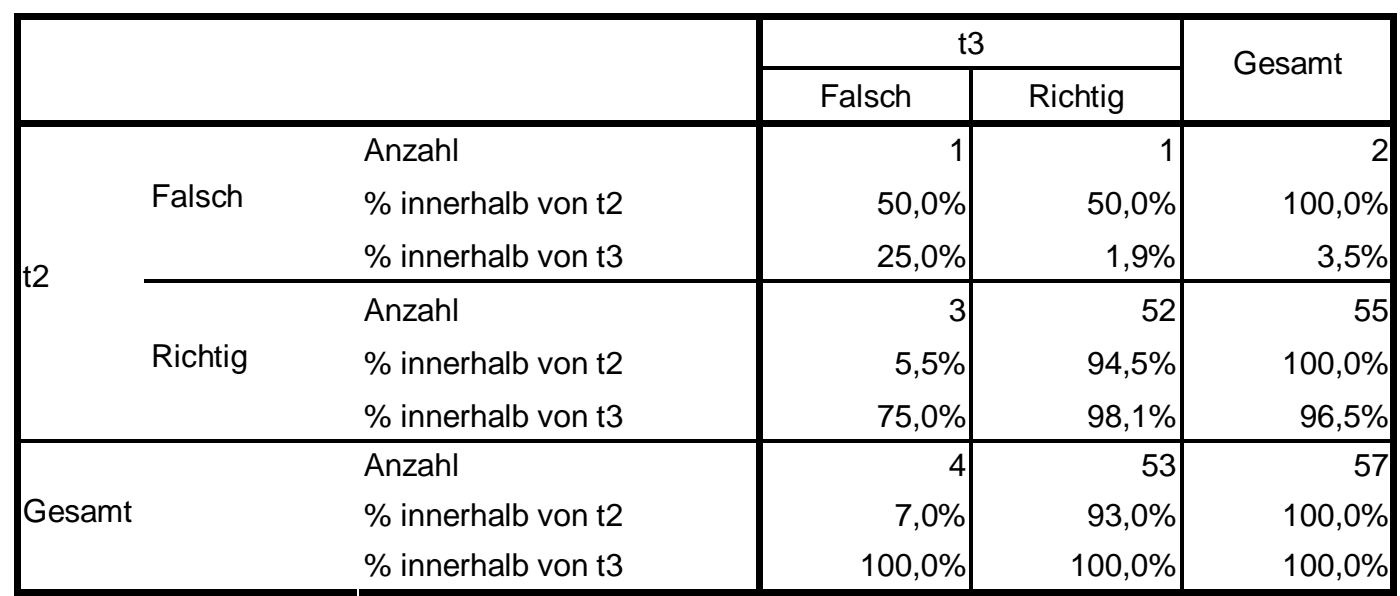

Tabelle A84: Ein Säugling mit Herzfrequenz unter 60/min muss sofort intubiert werden t2 vs. t3

\begin{tabular}{|l|r|c|}
\hline & Wert & $\begin{array}{c}\text { Exakte Signifikanz } \\
\text { (2-seitig) }\end{array}$ \\
\hline $\begin{array}{l}\text { McNemar-Test } \\
\text { Anzahl der gültigen Fälle }\end{array}$ & 57 & $\mathbf{0 , 6 2 5}$ \\
\hline
\end{tabular}

Tabelle A85: McNemar-Test 
9) Thoraxkompressionen sollten den Thorax 1/3 des Weges bis zum Rücken eindrücken

\begin{tabular}{|c|c|c|c|c|c|}
\hline & & & \multicolumn{2}{|c|}{ t3 } & \multirow{2}{*}{ Gesamt } \\
\hline & & & Falsch & Richtig & \\
\hline \multirow{6}{*}{ t2 } & \multirow{3}{*}{ Falsch } & Anzahl & 0 & 1 & 1 \\
\hline & & $\%$ innerhalb von $\mathrm{t} 2$ & $0,0 \%$ & $100,0 \%$ & $100,0 \%$ \\
\hline & & $\%$ innerhalb von t3 & $0,0 \%$ & $1,8 \%$ & $1,8 \%$ \\
\hline & \multirow{3}{*}{ Richtig } & Anzahl & 2 & 54 & 56 \\
\hline & & $\%$ innerhalb von t2 & $3,6 \%$ & $96,4 \%$ & $100,0 \%$ \\
\hline & & $\%$ innerhalb von t3 & $100,0 \%$ & $98,2 \%$ & $98,2 \%$ \\
\hline \multirow{3}{*}{\multicolumn{2}{|c|}{ Gesamt }} & Anzahl & 2 & 55 & 57 \\
\hline & & $\%$ innerhalb von $\mathrm{t} 2$ & $3,5 \%$ & $96,5 \%$ & $100,0 \%$ \\
\hline & & $\%$ innerhalb von t3 & $100,0 \%$ & $100,0 \%$ & $100,0 \%$ \\
\hline
\end{tabular}

Tabelle A86: Thoraxkompressionen sollten den Thorax 1/3 des Weges bis zum Rücken eindrücken t2 vs. t3

\begin{tabular}{|l|r|c|}
\hline & Wert & $\begin{array}{c}\text { Exakte Signifikanz } \\
\text { (2-seitig) }\end{array}$ \\
\hline $\begin{array}{l}\text { McNemar-Test } \\
\text { Anzahl der gültigen Fälle }\end{array}$ & 57 & $\mathbf{1 , 0 0 0}$ \\
\hline
\end{tabular}

Tabelle A87: McNemar-Test

10) Systeme, die zur Beatmung von Neugeborenen benutzt werden, sollten ein Überdruckventil von 30-40 cm Wassersäule besitzen

\begin{tabular}{|ll|r|r|r|}
\hline \multirow{2}{*}{} & & \multicolumn{2}{|c|}{$\mathrm{t}$ Gesamt } \\
\cline { 2 - 4 } & Anzahl & \multicolumn{1}{|c|}{ Falsch } & \multicolumn{1}{|c|}{ Richtig } & \\
\hline \multirow{3}{*}{ t2 } & \multirow{2}{*}{ Richtig } & 13 & 44 & 57 \\
& \% innerhalb von t2 & $22,8 \%$ & $77,2 \%$ & $100,0 \%$ \\
& \% innerhalb von t3 & $100,0 \%$ & $100,0 \%$ & $100,0 \%$ \\
\hline \multirow{2}{*}{ Gesamt } & Anzahl & 13 & 44 & 57 \\
& \% innerhalb von t2 & $22,8 \%$ & $77,2 \%$ & $100,0 \%$ \\
& \% innerhalb von t3 & $100,0 \%$ & $100,0 \%$ & $100,0 \%$ \\
\hline
\end{tabular}

Tabelle A88: Systeme, die zur Beatmung von Neugeborenen benutzt werden, sollten ein Überdruckventil von 30$40 \mathrm{~cm}$ Wassersäule besitzen t2 vs. t3

11) Alle Kinder, die bei Geburt intubiert werden, müssen auf eine Neugeborenenintensivstation übernommen werden

\begin{tabular}{|c|c|c|c|c|c|}
\hline & & & $\mathrm{t}^{2}$ & & Gecomt \\
\hline & & & Falsch & Richtig & \\
\hline & & Anzahl & 1 & 0 & 1 \\
\hline & Falsch & $\%$ innerhalb von $t 2$ & $100,0 \%$ &, $0 \%$ & $100,0 \%$ \\
\hline t? & & $\%$ innerhalb von $\mathrm{t} 3$ & $20,0 \%$ &, $0 \%$ & $1,8 \%$ \\
\hline & & Anzahl & 4 & 52 & 56 \\
\hline & Richtig & $\%$ innerhalb von $t 2$ & $7,1 \%$ & $92,9 \%$ & $100,0 \%$ \\
\hline & & $\%$ innerhalb von $\mathrm{t} 3$ & $80,0 \%$ & $100,0 \%$ & $98,2 \%$ \\
\hline & & Anzahl & 5 & 52 & 57 \\
\hline Gesamt & & $\%$ innerhalb von $t 2$ & $8,8 \%$ & $91,2 \%$ & $100,0 \%$ \\
\hline & & $\%$ innerhalb von $\mathrm{t} 3$ & $100,0 \%$ & $100,0 \%$ & $100,0 \%$ \\
\hline
\end{tabular}

Tabelle A89: Alle Kinder, die bei Geburt intubiert werden, müssen auf eine Neugeborenenintensivstation übernommen werden $\mathrm{t} 2$ vs. $\mathrm{t} 3$ 


\begin{tabular}{|l|r|r|}
\hline & Wert & $\begin{array}{c}\text { Exakte Signifikanz } \\
\text { (2-seitig) }\end{array}$ \\
\hline $\begin{array}{l}\text { McNemar-Test } \\
\text { Anzahl der gültigen Fälle }\end{array}$ & 57 & $\mathbf{0 , 1 2 5}$ \\
\hline
\end{tabular}

Tabelle A90: McNemar-Test

12) Hypothermie kann Apnoen verursachen

\begin{tabular}{|c|c|c|c|c|c|}
\hline & & & \multicolumn{2}{|c|}{ t3 } & \multirow{2}{*}{ Gesamt } \\
\hline & & & Falsch & Richtig & \\
\hline \multirow{6}{*}{ t2 } & \multirow{3}{*}{ Falsch } & Anzahl & 0 & 1 & 1 \\
\hline & & $\%$ innerhalb von t2 & $0,0 \%$ & $100,0 \%$ & $100,0 \%$ \\
\hline & & $\%$ innerhalb von $\mathrm{t} 3$ & $0,0 \%$ & $1,8 \%$ & $1,8 \%$ \\
\hline & \multirow{3}{*}{ Richtig } & Anzahl & 2 & 54 & 56 \\
\hline & & $\%$ innerhalb von $\mathrm{t} 2$ & $3,6 \%$ & $96,4 \%$ & $100,0 \%$ \\
\hline & & $\%$ innerhalb von t3 & $100,0 \%$ & $98,2 \%$ & $98,2 \%$ \\
\hline \multirow{3}{*}{\multicolumn{2}{|c|}{ Gesamt }} & Anzahl & 2 & 55 & 57 \\
\hline & & $\%$ innerhalb von t2 & $3,5 \%$ & $96,5 \%$ & $100,0 \%$ \\
\hline & & $\%$ innerhalb von t3 & $100,0 \%$ & $100,0 \%$ & $100,0 \%$ \\
\hline
\end{tabular}

Tabelle A91: Hypothermie kann Apnoen verursachen t2 vs. 13

\begin{tabular}{|l|r|r|}
\hline & Wert & $\begin{array}{c}\text { Exakte Signifikanz } \\
\text { (2-seitig) }\end{array}$ \\
\hline $\begin{array}{l}\text { McNemar-Test } \\
\text { Anzahl der gültigen Fälle }\end{array}$ & 57 & $\mathbf{1 , 0 0 0}$ \\
\hline
\end{tabular}

Tabelle A92: McNemar-Test

13) Es ist ratsam, die Reanimation bei einem Frühgeborenen mit einem niedrigeren Beatmungsdruck zu starten als bei einem reifen Neugeborenen

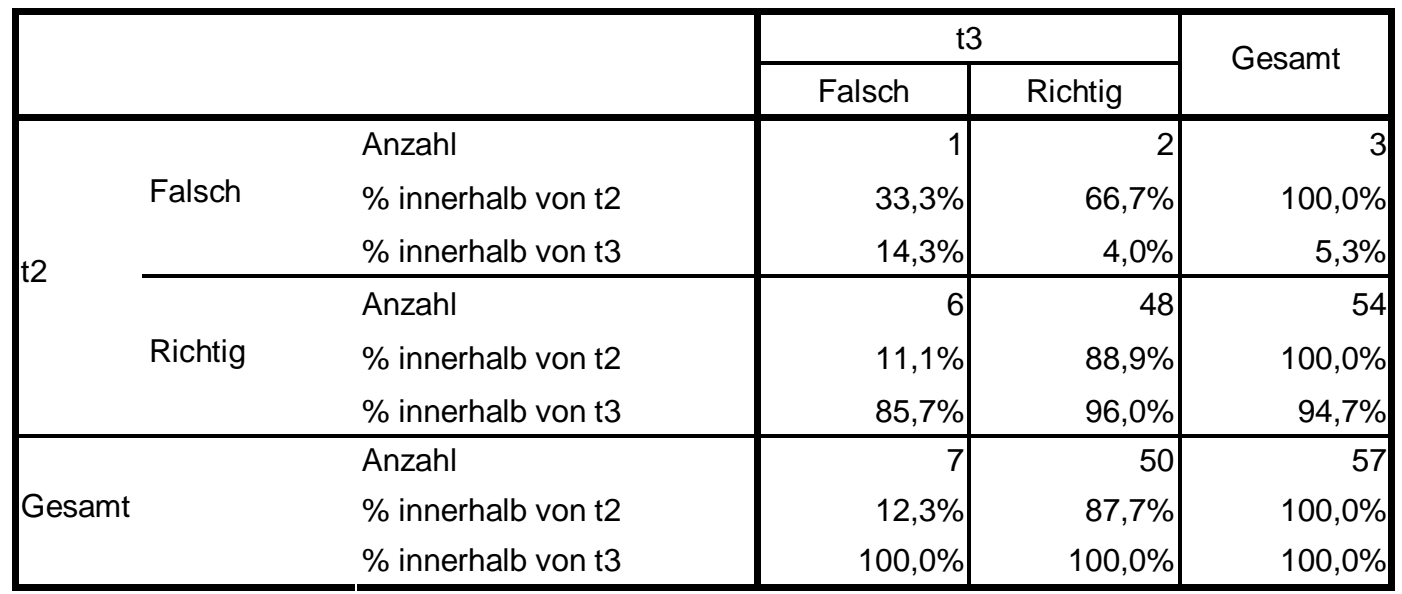

Tabelle A93: Es ist ratsam, die Reanimation bei einem Frühgeborenen mit einem niedrigeren Beatmungsdruck zu starten als bei einem reifen Neugeborenen t2 vs. $\mathrm{t} 3$

\begin{tabular}{|l|r|r|}
\hline & Wert & $\begin{array}{c}\text { Exakte Signifikanz } \\
\text { (2-seitig) }\end{array}$ \\
\hline $\begin{array}{l}\text { McNemar-Test } \\
\text { Anzahl der gültigen Fälle }\end{array}$ & 57 & $\mathbf{0 , 2 8 9}$ \\
\hline
\end{tabular}

Tabelle A94: McNemar-Test 
14) Maskenbeatmung ist viel weniger effektiv als Beatmung nach Intubation

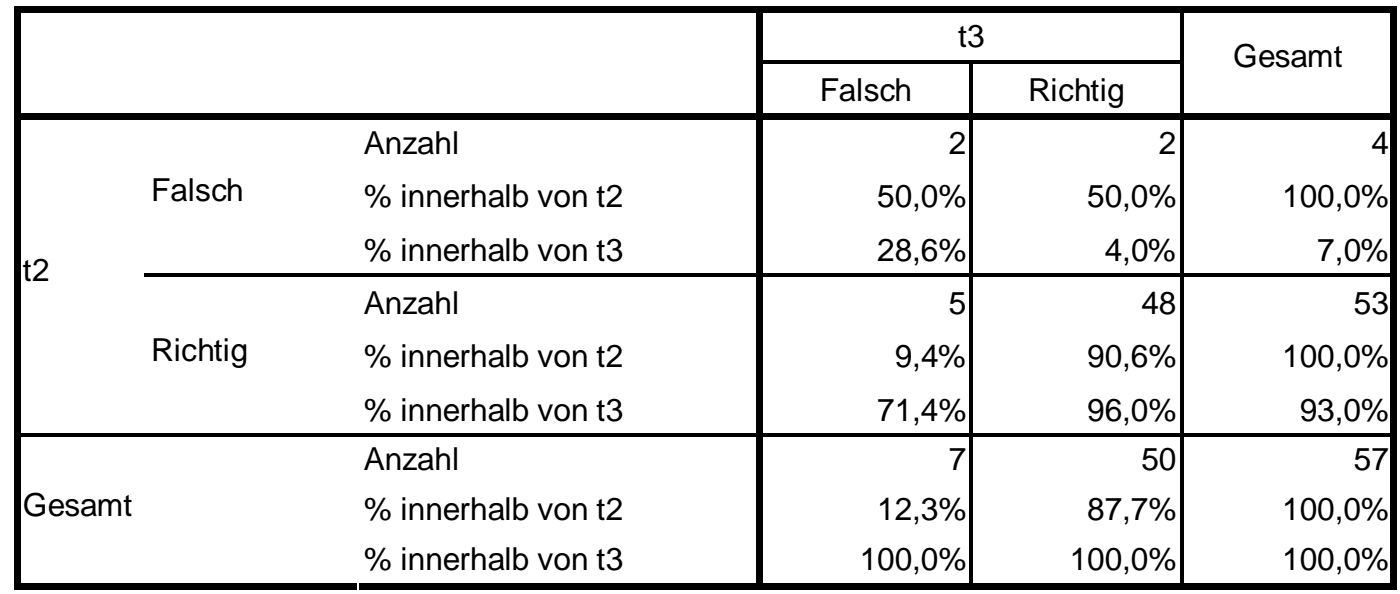

Tabelle A95: Maskenbeatmung ist viel weniger effektiv als Beatmung nach Intubation t2 vs. t3

\begin{tabular}{|l|r|c|}
\hline & Wert & $\begin{array}{c}\text { Exakte Signifikanz } \\
\text { (2-seitig) }\end{array}$ \\
\hline $\begin{array}{l}\text { McNemar-Test } \\
\text { Anzahl der gültigen Fälle }\end{array}$ & 57 & $\mathbf{0 , 4 5 3}$ \\
\hline
\end{tabular}

Tabelle A96: McNemar-Test

15) Die initiale Reanimation eines Frühgeborenen unterscheidet sich sehr von einer Reanimation bei einem Reifgeborenen

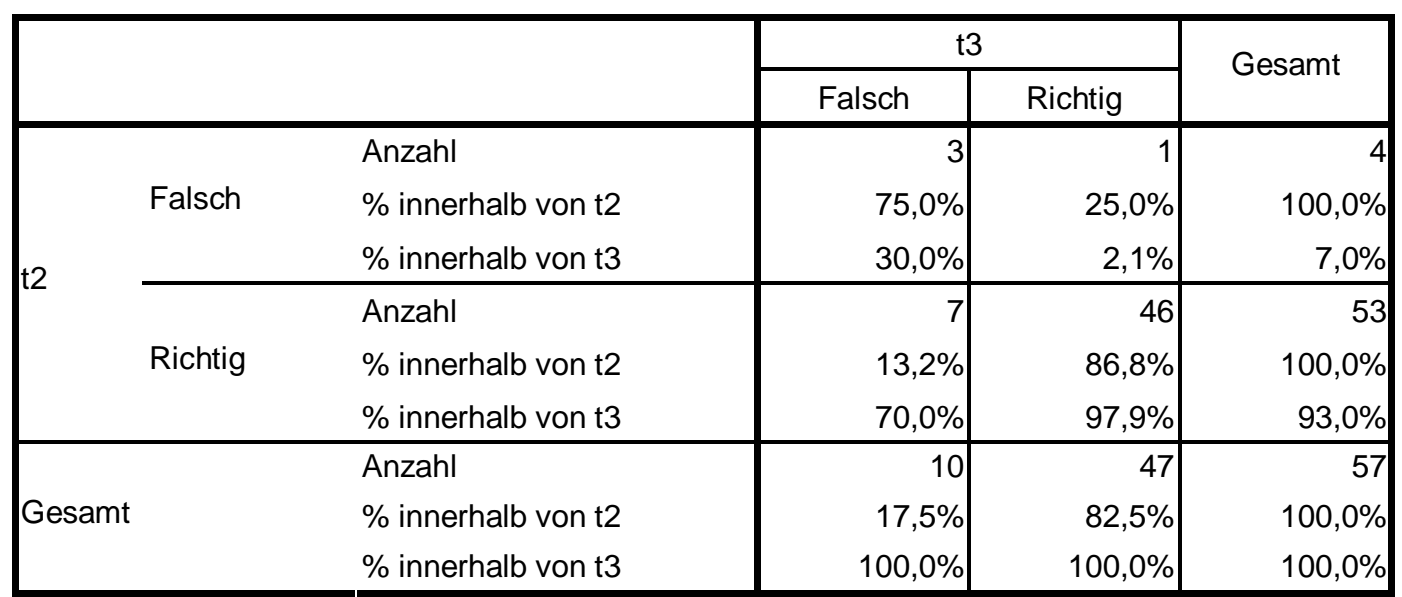

Tabelle A97: Die initiale Reanimation eines Frühgeborenen unterscheidet sich sehr von einer Reanimation bei einem Reifgeborenen $\mathrm{t} 2$ vs. $\mathrm{t} 3$

\begin{tabular}{|l|r|c|}
\hline & Wert & $\begin{array}{c}\text { Exakte Signifikanz } \\
\text { (2-seitig) }\end{array}$ \\
\hline $\begin{array}{l}\text { McNemar-Test } \\
\text { Anzahl der gültigen Fälle }\end{array}$ & 57 & $\mathbf{0 , 0 7 0}$ \\
\hline
\end{tabular}

Tabelle A98: McNemar-Test 
16)

Schnappatmung ist immer flach

\begin{tabular}{|c|c|c|c|c|c|}
\hline \multicolumn{5}{|c|}{ t3 } & \multirow{2}{*}{ Gesamt } \\
\hline & & & Falsch & Richtig & \\
\hline \multirow{6}{*}{ t2 } & \multirow{3}{*}{ Falsch } & Anzahl & 6 & 7 & 13 \\
\hline & & $\%$ innerhalb von $\mathrm{t} 2$ & $46,2 \%$ & $53,8 \%$ & $100,0 \%$ \\
\hline & & $\%$ innerhalb von t3 & $37,5 \%$ & $17,1 \%$ & $22,8 \%$ \\
\hline & \multirow{3}{*}{ Richtig } & Anzahl & 10 & 34 & 44 \\
\hline & & $\%$ innerhalb von $\mathrm{t} 2$ & $22,7 \%$ & $77,3 \%$ & $100,0 \%$ \\
\hline & & $\%$ innerhalb von $\mathrm{t} 3$ & $62,5 \%$ & $82,9 \%$ & $77,2 \%$ \\
\hline \multirow{3}{*}{\multicolumn{2}{|c|}{ Gesamt }} & Anzahl & 16 & 41 & 57 \\
\hline & & $\%$ innerhalb von t2 & $28,1 \%$ & $71,9 \%$ & $100,0 \%$ \\
\hline & & $\%$ innerhalb von t3 & $100,0 \%$ & $100,0 \%$ & $100,0 \%$ \\
\hline
\end{tabular}

Tabelle A99: Schnappatmung ist immer flach t2 vs. t3

\begin{tabular}{|l|r|c|}
\hline & Wert & $\begin{array}{c}\text { Exakte Signifikanz } \\
\text { (2-seitig) }\end{array}$ \\
\hline $\begin{array}{l}\text { McNemar-Test } \\
\text { Anzahl der gültigen Fälle }\end{array}$ & 57 & $\mathbf{0 , 6 2 9}$ \\
\hline
\end{tabular}

Tabelle A100: McNemar-Test

17) Nach einer Reanimation ist wichtig: Abnahme von Nabelschnurblut und venösem Blut zur Messung von $\mathrm{pH}$ und $\mathrm{BE}$

\begin{tabular}{|c|c|c|c|c|c|}
\hline & & & \multicolumn{2}{|c|}{ t3 } & \multirow{2}{*}{ Gesamt } \\
\hline & & & Falsch & Richtig & \\
\hline \multirow{6}{*}{ t2 } & \multirow{3}{*}{ Falsch } & Anzahl & 2 & 1 & 3 \\
\hline & & $\%$ innerhalb von t2 & $66,7 \%$ & $33,3 \%$ & $100,0 \%$ \\
\hline & & $\%$ innerhalb von $\mathrm{t} 3$ & $28,6 \%$ & $2,0 \%$ & $5,3 \%$ \\
\hline & \multirow{3}{*}{ Richtig } & Anzahl & 5 & 49 & 54 \\
\hline & & $\%$ innerhalb von t2 & $9,3 \%$ & $90,7 \%$ & $100,0 \%$ \\
\hline & & $\%$ innerhalb von t3 & $71,4 \%$ & $98,0 \%$ & $94,7 \%$ \\
\hline \multirow{3}{*}{\multicolumn{2}{|c|}{ Gesamt }} & Anzahl & 7 & 50 & 57 \\
\hline & & $\%$ innerhalb von t2 & $12,3 \%$ & $87,7 \%$ & $100,0 \%$ \\
\hline & & $\%$ innerhalb von t3 & $100,0 \%$ & $100,0 \%$ & $100,0 \%$ \\
\hline
\end{tabular}

Tabelle A101: Nach einer Reanimation ist wichtig: Abnahme von Nabelschnurblut und venösem Blut zur Messung von $\mathrm{pH}$ und $\mathrm{BE}$ t2 vs. $\mathrm{t} 3$

\begin{tabular}{|l|c|c|}
\hline & Wert & $\begin{array}{c}\text { Exakte Signifikanz } \\
\text { (2-seitig) }\end{array}$ \\
\hline $\begin{array}{l}\text { McNemar-Test } \\
\text { Anzahl der gültigen Fälle }\end{array}$ & 57 & $\mathbf{0 , 2 1 9}$ \\
\hline
\end{tabular}

Tabelle A102: McNemar-Test 


\section{Anhang 7.9 Berufsgruppenbezogener Vergleich der Gesamtstatistik}

\section{1) Vergleich der Scores zwischen den Berufsgruppen (t1 und t2: 170 Fälle, t3: 53 Fälle)}

a) Vor Schulung (\%) bei den verschiedenen Berufsgruppen

\begin{tabular}{|l|r|r|r|r|r|}
\hline Berufsgruppe & Mittelwert (\%) & $\begin{array}{c}\text { Standard- } \\
\text { abweichung (\%) }\end{array}$ & $\begin{array}{c}\text { Standardfehler des } \\
\text { Mittelwertes (\%) }\end{array}$ & Median(\%) & $\mathrm{N}$ \\
\hline Gynäkologe & 82,18 & 9,873 & 1,693 & 82,35 & 34 \\
Pädiater & 87,71 & 8,302 & 1,238 & 88,24 & 45 \\
Anästhesist & 82,35 & 9,843 & 1,930 & 82,35 & 26 \\
Hebamme & 76,29 & 9,918 & 1,727 & 76,47 & 33 \\
Kinderkrankenschwester & 74,82 & 10,377 & 1,834 & 76,47 & 32 \\
Insgesamt & 81,14 & 10,668 & 0,818 & 82,35 & 170 \\
\hline
\end{tabular}

Tabelle A103: Vor Schulung (\%) bei den verschiedenen Berufsgruppen

\begin{tabular}{|l|r|}
\hline & Vor Schulung (\%) \\
\hline Chi-Quadrat & 36,102 \\
Df & 4 \\
Asymptotische Signifikanz & $\mathbf{0 , 0 0 0}$ \\
\hline
\end{tabular}

Tabelle A104: H-Test nach Kruskal und Wallis; Gruppenvariable: Berufsgruppe

b) Direkt nach dem Kurs (\%) bei den verschiedenen Berufsgruppen

\begin{tabular}{|l|r|r|r|r|r|}
\hline Berufsgruppe & Mittelwert (\%) & $\begin{array}{c}\text { Standard- } \\
\text { abweichung (\%) }\end{array}$ & $\begin{array}{c}\text { Standardfehler des } \\
\text { Mittelwertes (\%) }\end{array}$ & Median (\%) & $\mathrm{N}$ \\
\hline Gynäkologe & 94,12 & 5,971 & 1,024 & 94,12 & 34 \\
Pädiater & 96,60 & 3,863 & 0,576 & 100,00 & 45 \\
Anästhesist & 93,44 & 5,081 & 0,997 & 94,12 & 26 \\
Hebamme & 87,88 & 10,496 & 1,827 & 88,24 & 33 \\
Kinderkrankenschwester & 87,50 & 10,852 & 1,918 & 88,24 & 32 \\
Insgesamt & 92,21 & 8,431 & 0,647 & 94,12 & 170 \\
\hline
\end{tabular}

Tabelle A105: Direkt nach dem Kurs (\%) bei den verschiedenen Berufsgruppen

\begin{tabular}{|l|r|}
\hline & Direkt nach Schulung (\%) \\
\hline Chi-Quadrat & 28,701 \\
Df & 4 \\
Asymptotische Signifikanz & $\mathbf{0 , 0 0 0}$ \\
\hline
\end{tabular}

Tabelle A106: H-Test nach Kruskal und Wallis; Gruppenvariable: Berufsgruppe 
c) Nach 6 Monaten (\%) bei den verschiedenen Berufsgruppen

\begin{tabular}{|l|r|r|r|r|r|}
\hline Berufsgruppe & Mittelwert (\%) & $\begin{array}{c}\text { Standard- } \\
\text { abweichung (\%) }\end{array}$ & $\begin{array}{c}\text { Standardfehler des } \\
\text { Mittelwertes (\%) }\end{array}$ & Median (\%) & $\mathrm{N}$ \\
\hline Gynäkologe & 88,24 & 14,003 & 5,293 & 88,24 & 7 \\
Pädiater & 92,94 & 5,070 & 1,309 & 94,12 & 15 \\
Anästhesist & 85,29 & 3,108 & 6,310 & 88,24 & 6 \\
Hebamme & 85,47 & 7,814 & 1,895 & 82,35 & 17 \\
Kinderkrankenschwester & 77,21 & 7,332 & 2,592 & 76,47 & 8 \\
Insgesamt & 86,68 & 9,383 & 1,289 & 88,24 & 53 \\
\hline
\end{tabular}

Tabelle A107: Nach 6 Monaten (\%) bei den verschiedenen Berufsgruppen

\begin{tabular}{|l|r|}
\hline & \multicolumn{1}{|r|}{ Nach 6 Monaten (\%) } \\
\hline Chi-Quadrat & 17,684 \\
Df & 4 \\
Asymptotische Signifikanz & $\mathbf{0 , 0 0 1}$ \\
\hline
\end{tabular}

Tabelle A108: H-Test nach Kruskal und Wallis; Gruppenvariable: Berufsgruppe 


\section{Literaturverzeichnis}

AHA (American Heart Association; American Academy of Pediatrics) (2005): 2005 American Heart Association (AHA) guidelines for cardiopulmonary resuscitation (CPR) and emergency cardiovascular care (ECC) of pediatric and neonatal patients: neonatal resuscitation guidelines. Circulation 112(24) IV188-195

ÄKN (Zentrum für Qualität und Management im Gesundheitswesen, Einrichtung der Ärztekammer Niedersachsen) (2007): Jahresauswertung 2007 Qualitätsmanagement Geburtshilfe, Modul 16/1, www.zq-aekn.de, Abruf vom 02.11.2009

Aubrey WR, Yoxall CW (2001): Evaluation of the role of the neonatal nurse practitioner in resuscitation of preterm infants at birth. Arch Dis Child Fetal Neonatal Ed 85(2) F96-9

Aziz K, Chadwick M, Downton G, Baker M, Andrews W (2005): The development and implementation of a multidisciplinary neonatal resuscitation team in a Canadian perinatal centre. Resuscitation $\underline{66} 45-51$

Baskett PJ, Nolan JP, Handley A, Soar J, Biarent D, Richmond S; European Resuscitation Council (2005): European Resuscitation Council guidelines for resuscitation 2005. Resuscitation. 67(1) 3-189

Bould MD, Hayter MA, Campbell DM, Chandra DB, Joo HS, Naik VN (2009): Cognitive aid for neonatal resuscitation: a prospective single-blinded randomized controlled trial. Br J Anaesth 103(4) 570-5

Bryce J, Boschi-Pinto C, Shibuya K, Black RE; WHO Child Health Epidemiology Reference Group (2005): WHO estimates of the causes of death in children. Lancet $\underline{1 ; 365(9465)} 1147-52$

Carcillo JA, Kuch BA, Han YY, Day S, Greenwald BM, McCloskey KA, PearsonShaver AL, Orr RA (2009): Mortality and functional morbidity after use of PALS/APLS by community physicians. Pediatrics $\underline{124(2)}, 500-8$ 
Chamberlain D, Cummins RO, Montgomery WH, Kloeck WG, Nadkarni VM (2005): International collaboration in resuscitation medicine. Resuscitation $\underline{67(2-3)}$ 163-5

Chan LC, Hey E (2006): Can all neonatal resuscitation be managed by nurse practitioners? Arch Dis Child Fetal Neonatal Ed 91(1) 52-5

Cooke JM, Larsen J, Hamstra SJ, Andreatta PB (2008): Simulation enhances resident confidence in critical care and procedural skills. Fam Med 40(3) 165-7

Dunn S, Niday P, Watters NE, McGrath P, Alcock D (1992): The provision and evaluation of a neonatal resuscitation program. J Contin Educ Nurs $\underline{23(3)}, 118-26$

Duran R, Aladağ N, Vatansever U, Süt N, Acunaş B (2008 a): The impact of Neonatal Resuscitation Program courses on mortality and morbidity of newborn infants with perinatal asphyxia. Brain Dev $\underline{30(1)}$, 43-6

Duran R, Aladağ N, Vatansever U, Küçükuğurluoğlu Y, Süt N, Acunaş B (2008 b): Proficiency and knowledge gained and retained by pediatric residents after neonatal resuscitation course. Pediatr Int 50(5), 644-7

Ergenekon E, Koç E, Atalay Y, Soysal S (2000): Neonatal resuscitation course experience in Turkey. Resuscitation. 1;45(3) 225-7

Finer NN, Rich W (2002): Neonatal resuscitation: toward improved performance. Resuscitation $\underline{\text { 53(1) } 47-51}$

Foster K, Craven P, Reid S (2006): Neonatal resuscitation educational experience of staff in New South Wales and Australian Capital Territory hospitals. J Paediatr Child Health $\underline{42(1-2)}$ 16-9 
Joint Commission on Accreditation of Healthcare Organisations (2004): Preventing infant death and injury during delivery. Sentinel Event Alert (30) 1-3; siehe auch http://www.jointcommission.org/SentinelEvents/SentinelEventAlert/sea 30.htm, Abruf vom 01.12.2009

Jukkala AM, Henly SJ (2009): Provider readiness for neonatal resuscitation in rural hospitals. J Obstet Gynecol Neonatal Nurs $\underline{38(4)}$ 443-52

Kattwinkel J: Neonatal resuscitation textbook $5^{\text {th }}$ ed. American Heart Association, American Academy of Pediatrics, Dallas 2006

Kattwinkel J (2008): Neonatal resuscitation guidelines for ILCOR and NRP: evaluating the evidence and developing a consensus. J Perinatol $\underline{28}(3)$ 27-9

Levitt C, Kaczorowski J, Outerbridge E, Jimenez V, Connolly B, Slapcoff B (1996): Knowledge gained following Neonatal Resuscitation Program courses. Fam Med $\underline{28(6)}, 403-6$

Lorenz N, GliniorzC, Nordwig A, Rothe KF, Kabus M (2007): Dresdner Kurs Basale Neugeborenenreanimation. Ärzteblatt Sachsen 18 (1): 20-25, siehe auch www.khdf.de/khdf/kliniken/kai/extern/akn2001/html/neugeborenen-reanimation.html, Abruf vom 05.11.2009

Mitchell A, Niday P, Boulton J, Chance G, Dulberg C (2002): A prospective clinical audit of neonatal resuscitation practices in Canada. Adv Neonatal Care 2(6)316-26

Palme-Kilander C (1992): Methods of resuscitation in low-Apgar-score newborn infants--a national survey. Acta Paediatr 81(10) 739-44

Patel D, Piotrowski ZH, Nelson MR, Sabich R (2001): Effect of a statewide neonatal resuscitation training program on Apgar scores among high-risk neonates in Illinois. Pediatrics 107(4), 648-55 
Pederzini F, Rugolotto S,Wermter B, Cologna M, Malossi R, Morandell T, Perriccelli M, Oberammer R, Pasqualoni B, Schellenberg S (2008): Newborn life support courses (ERC) in a bilingual area where newborn resuscitation program courses (AHA) are taught as well. Resuscitation $\underline{77(0)} 58$

Raupp P, McCutcheon C (2007): Neonatal resuscitation - An analysis of the transatlantic divide. Resuscitation $\underline{75(2)}$, 345-9

Richmond S: Resuscitation at birth - Newborn life support provider course manual $2^{\text {nd }}$ ed. Resuscitation Council, Edegem/Belgium 2008

Rogers PL, Jacob H, Rashwan AS, Pinsky MR (2001): Quantifying learning in medical students during a critical care medicine elective: a comparison of three evaluation instruments. Crit Care Med 29(6) 1268-73

Russo SG, Eich C, Barwing J, Nickel EA, Braun U, Graf BM, Timmermann A (2007): Self-reported changes in attitude and behavior after attending a simulation-aided airway management course. J Clin Anesth 19(7) 517-22

Ryan CA, Ahmed S, Abdullah H, McCarthy-Clark L, Malone A (1998): Dissemination and evaluation of AAP/AHA Neonatal Resuscitation Programme in Ireland. Ir Med J 91(2) $51-2$

Sayre MR, Sakles JC, Mistler AF, Evans JL, Kramer AT, Pancioli AM (1998): Field trial of endotracheal intubation by basic EMTs. Ann Emerg Med $\underline{31(2)}$ 228-33

Simpson KR, James DC, Knox GE (2006): Nurse-physician communication during labor and birth: implications for patient safety. J Obstet Gynecol Neonatal Nurs 35(4) 547-56

Singh J, Santosh S, Wyllie JP, Mellon A (2006): Effects of a course in neonatal resuscitation--evaluation of an educational intervention on the standard of neonatal resuscitation. Resuscitation $\underline{68(3)}$ 385-9 
SLÄK (Projektgeschäftsstelle Qualitätssicherung bei der Sächsischen Landesärztekammer) (2008): Qualitätsbericht Geburtshilfe Jahresauswertung 2008, Modul 16/1, www.slaek.de/36quali/80Downloads/2008/16n1.pdf, Abruf vom 02.11 .2009

Sørensen JL, Løkkegaard E, Johansen M, Ringsted C, Kreiner S, McAleer S (2009): The implementation and evaluation of a mandatory multi-professional obstetric skills training program. Acta Obstet Gynecol Scand 17 1-11

Trevisanuto D, Ferrarese P, Cavicchioli P, Fasson A, Zanardo V, Zacchello F (2005): Knowledge gained by pediatric residents after neonatal resuscitation program courses. Paediatr Anaesth 15(11) 944-7

Verplancke T, De Paepe P, Calle PA, De Regge M, Van Maele G, Monsieurs KG (2008): Determinants of the quality of basic life support by hospital nurses. Resuscitation $\underline{77(1)}$ 75-80

Walker DE, Balvert L (1994): A practical program to maintain neonatal resuscitation skills. CMAJ 151(3) 299-304

WHO (2005): World health report 2005: make every mother and child count. WHO Press Genf/Schweiz 2005, www.who.int/whr/2005/whr2005 en.pdf Abruf vom 05.11.2009 


\section{Danksagung}

Ich möchte Dr. U. Kreth (dr.wiki) für die gute Zusammenarbeit und die Unterstützung bei der Erhebung der Daten danken. Ich danke ebenfalls Frau Dr. T. Kottmann (Statistik-Service/Medizinische Wissenschaftsberatung) für die statistische Beratung und Prof. Dr. J.-H. Schiffmann für die gute Betreuung dieser Arbeit.

Kathrin Hanke

Frankfurt, 3. Januar 2010 


\section{Lebenslauf}

\section{Beruflicher Werdegang}

Am 20.12.1976 wurde ich in Bad Gandersheim geboren.

Nach dem Abitur im Roswitha-Gymnasium Bad Gandersheim 1996 begann ich das Studium der Humanmedizin an der Georg-August-Universität Göttingen im August 1996. Im September 1998 absolvierte ich dort das Physikum, im September 1999 das Erste Staatsexamen. Nach einem Auslandssemester von August 2000 bis Februar 2001 in Spanien legte ich im April 2002 erfolgreich das 2. Staatsexamen an der Georg- AugustUniversität Göttingen ab.

Nach dem Praktischen Jahr schloss ich im April 2003 das Medizinstudium mit dem dritten Staatsexamen in dem, der Georg-August-Universität Göttingen angeschlossenen, St. Bernward Krankenhaus in Hildesheim ab.

Im August 2003 begann ich eine Tätigkeit als Arzt im Praktikum in der pädiatrischen Abteilung des Klinikums Niederberg in Velbert, wo ich bis zum April 2006 beschäftigt blieb. Im Oktober 2004 erhielt ich meine Approbation.

Im Mai 2006 begann ich meine ärztliche Tätigkeit in der pädiatrischen Abteilung des Lukaskrankenhaus Neuss. Dort war ich bis Dezember 2008 beschäftigt.

Im Oktober 2008 legte ich die Prüfung zur Fachärztin für Kinder- und Jugendheilkunde ab.

Seit März 2009 bin ich in der anästhesiologischen Abteilung des Markus-Krankenhauses in Frankfurt am Main beschäftigt.

\section{Zusätzliche ärztliche Tätigkeit}

Seit Januar 2008 bin ich aktiv als Ausbilder des ERC (European Resuscitation Council) bei Kursen für Kinder- und Neugeborenenreanimation

Zwischen Oktober und Dezember 2005 nahm ich an einem freiwilligen Aufenthalt in Managua/Nicaragua im Rahmen der Organisation „Ärzte für die dritte Welt“ teil.

\section{Persönliches}

Ich bin mit Dr. Jörg Hanke verheiratet, wir haben eine gemeinsame Tochter. 